\title{
Solution-Phase Synthesis of Nucleobase-
}

\section{Substituted Analogs of Triostin A}

\author{
Katrin B. Lorenz and Ulf Diederichsen* \\ Institut für Organische und Biomolekulare Chemie, Georg-August Universität Göttingen, \\ Tammannstraße 2, D-37077 Göttingen. \\ udieder@gwdg.de
}

\section{Supporting Information}

General Experimental............................................................. 3

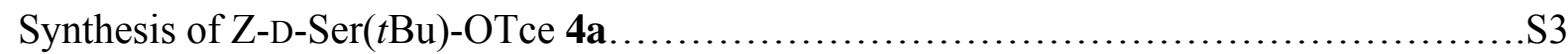

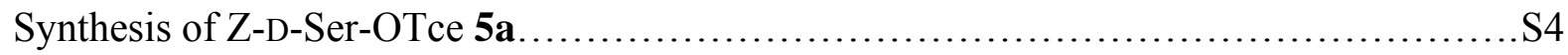

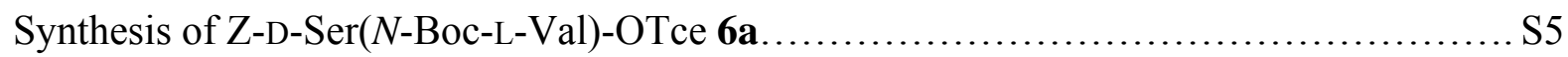

Synthesis of (Z-D-Ser-L-Ala-L-Cys-L-Val) 2 (serine hydroxyl) dilactone disulfide 14a....... S6

\section{NMR Spectra}

4a.

$5 a$

$6 a$ S11

7a $\mathrm{S} 13$

$8 a$

$10 a$ S21

11a. S25 
13a. S33

$14 a$. S37

15 S41

16 S44

4b S48

$5 b$ S50

$6 b$ S52

$7 b$ S54

$8 b$ S57

$10 b$ S61

11b S64

12b S68

13b S72

14b S76

17 S80 


\section{General experimental}

Solvents were of the highest grade available. Pyridine and dichloromethane were distilled from calcium hydride prior to use. DMF was purchased dry and stored over $4 \AA$ molecular sieves. Commercially available reagents were of analytical grade and used without further purification. Melting points are uncorrected. IR spectra were recorded using $\mathrm{KBr}$ pellets. Chemical shifts are referenced to the residual solvent peaks of $\mathrm{CDCl}_{3}\left({ }^{1} \mathrm{H}: \delta=7.24 \mathrm{ppm},{ }^{13} \mathrm{C}\right.$ : $\delta=77.0 \mathrm{ppm})$ or DMSO- $d_{6}\left({ }^{1} \mathrm{H}: \delta=2.49 \mathrm{ppm},{ }^{13} \mathrm{C}: \delta=39.5 \mathrm{ppm}\right)$. NMR assignments were made using APT as well as HMQC and ${ }^{1} \mathrm{H}^{1} \mathrm{H}-\mathrm{COSY}$ data. HPLC was performed using ODSH80/RP-C18 columns for analytical samples $(250 \times 4.6 \mathrm{~mm}, 5 \mu \mathrm{m}, 120 \AA)$ and preparative runs $(250 \times 20 \mathrm{~mm}, 5 \mu \mathrm{m}, 120 \AA)$. Flash chromatography was performed on silica gel 60 , 0.040-0.063 mm. Analytical thin-layer chromatography was visualized with UV light (254 $\mathrm{nm})$ or by dyeing with ninhydrine (3\% in ethanol).

Z-D-Ser(tBu)-OTce 4a. ${ }^{13}$ A solution of Z-D-Ser( $\left.t \mathrm{Bu}\right)-\mathrm{OH}(\mathbf{3 a} ; 15 \mathrm{~g}, 50.8 \mathrm{mmol})$ in pyridine $(150 \mathrm{~mL})$ was cooled to $-10{ }^{\circ} \mathrm{C}$ and treated sequentially with $\mathrm{HOBt}(11.7 \mathrm{~g}, 76.2 \mathrm{mmol}, 1.5$ equiv.) and Tce-OH (6.35 mL, $66.0 \mathrm{mmol}, 1.3$ equiv.). After $5 \mathrm{~min}$, solid DCC (15.7 g, 76.2 mmol, 1.5 equiv.) was added in small portions and the mixture was stirred for $18 \mathrm{~h}$ at $0{ }^{\circ} \mathrm{C}$. The precipitated DCU was separated by filtration, the filtrate was evaporated in vacuo and the residue was diluted with ethyl acetate $(200 \mathrm{~mL})$. The solution was washed consecutively with cold $1 \mathrm{~N}$ aqueous $\mathrm{HCl}(2 \times 200 \mathrm{~mL}), 5 \%$ aqueous $\mathrm{NaHCO}_{3}(200 \mathrm{~mL})$, and saturated aqueous $\mathrm{NaCl}(150 \mathrm{~mL})$, dried $\left(\mathrm{MgSO}_{4}\right)$, filtered and concentrated in vacuo. Flash chromatography $\left(\mathrm{SiO}_{2}, 8 \times 20 \mathrm{~cm}, 20 \%\right.$ ethyl acetate-hexane) afforded 4a (13.2 g, $\left.30.9 \mathrm{mmol}, 61 \%\right)$ as syrupy residue, which solidified upon prolonged standing, and Z-D-Ser $(t \mathrm{Bu})-\mathrm{OH}(3.72 \mathrm{~g}, 12.6$ mmol), which was reisolated and subjected to the same procedure, resulting in an overall yield of $78 \%$ 4a $(16.8 \mathrm{~g}, 39.5 \mathrm{mmol})$ as white solid: TLC (20\% ethyl acetate/hexane) $R_{f} 0.54$; $[\alpha]^{25}{ }_{\mathrm{D}}+12(\mathrm{c} 0.22, \mathrm{MeOH}) ; \operatorname{mp} 69-71{ }^{\circ} \mathrm{C} ; \mathrm{UV}(\mathrm{MeOH}) \lambda_{\max }(\varepsilon) 264 \mathrm{~nm}$; IR $(\mathrm{KBr}) v_{\max } 3379$, 
2976, 2938, 1749, 1722, 1520, 1364, 1288, 1264, 1232, 1197, 1171, 1104, 1088, 1069, 1049, 794, 742, $722 \mathrm{~cm}^{-1} ;{ }^{1} \mathrm{H}$ NMR $\left(\mathrm{CDCl}_{3}, 300 \mathrm{MHz}\right) \delta 1.16\left(\mathrm{~s}, 9 \mathrm{H}, t \mathrm{Bu}-\mathrm{CH}_{3}\right), 3.61\left(\mathrm{dd}, 1 \mathrm{H},{ }^{2} J=3\right.$ $\left.\mathrm{Hz},{ }^{3} J=9 \mathrm{~Hz}, \operatorname{Ser}-\mathrm{H} \beta\right), 3.93\left(\mathrm{dd}, 1 \mathrm{H},{ }^{2} J=3 \mathrm{~Hz},{ }^{3} J=9 \mathrm{~Hz}, \operatorname{Ser}-\mathrm{H} \beta\right), 4.56-4.63$ (m, 1H, Ser$\mathrm{H \alpha}), 4.70\left(\mathrm{~d}, 1 \mathrm{H},{ }^{2} \mathrm{~J}=12 \mathrm{~Hz}, \mathrm{Tce}-\mathrm{CH}_{2}\right), 4.81\left(\mathrm{~d}, 1 \mathrm{H},{ }^{2} \mathrm{~J}=12 \mathrm{~Hz}\right.$, Tce-CH$), 5.17(\mathrm{~s}, 2 \mathrm{H}, \mathrm{Z}-$ $\left.\mathrm{CH}_{2}\right), 5.68\left(\mathrm{~d}, 1 \mathrm{H},{ }^{3} \mathrm{~J}=9 \mathrm{~Hz}, \mathrm{Ser}-\mathrm{NH}\right), 7.35-7.41$ (m, 5H, Z-aromat. $\left.\mathrm{CH}\right) ;{ }^{13} \mathrm{C} \mathrm{NMR}\left(\mathrm{CDCl}_{3}\right.$, $63 \mathrm{MHz}) \delta 27.3\left(t \mathrm{Bu}-\mathrm{CH}_{3}\right), 54.5(\mathrm{Ser}-\mathrm{CH} \alpha), 61.6\left(\mathrm{Ser}_{-} \mathrm{CH}_{2} \beta\right), 67.2\left({\left.\mathrm{Z}-\mathrm{CH}_{2}\right),}\right), 6(t \mathrm{Bu}-$ quart. C), 74.7 (Tce- $\left.\mathrm{CH}_{2}\right), 94.3\left(\right.$ Tce- $\left.\mathrm{CCl}_{3}\right), 128.2,128.6$ (Z-aromat. $\mathrm{CH}$ ), 136.0 (Z-quart. C), 156.2 (Z-CO), 169.4 (CO); ESI-MS ( $\left.\mathrm{H}_{2} \mathrm{O} / \mathrm{ACN} / \mathrm{TFA}\right) \mathrm{m} / \mathrm{z} 371\left([\mathrm{M}-\mathrm{tBu}+\mathrm{H}]^{+}, 20\right), 427\left([\mathrm{M}+\mathrm{H}]^{+}\right.$, 100).

Z-D-Ser-OTce 5a. ${ }^{13}$ Compound 4a $(13.2$ g, 30.9 mmol) was treated with TFA $(70 \mathrm{~mL})$ and the mixture was stirred at $10{ }^{\circ} \mathrm{C}$ for $45 \mathrm{~min}$ before the TFA was removed in vacuo. The residue was dissolved in ethyl acetate $(200 \mathrm{~mL})$, washed with $5 \%$ aqueous $\mathrm{NaHCO}_{3}(200 \mathrm{~mL})$ and saturated aqueous $\mathrm{NaCl}(150 \mathrm{~mL})$, dried $\left(\mathrm{MgSO}_{4}\right)$, filtered and concentrated in vacuo. Purification by flash chromatography $\left(\mathrm{SiO}_{2}, 7 \times 18 \mathrm{~cm}, 33 \%\right.$ ethyl acetate-hexane) furnished 5a (8.70 g, $23.5 \mathrm{mmol}, 76 \%)$ as syrupy residue, which crystallized upon prolonged standing: TLC (35\% ethyl acetate/hexane) $R_{f} 0.24 ;[\alpha]^{25}+14$ (c $\left.0.13, \mathrm{MeOH}\right) ; \mathrm{mp} 93-95{ }^{\circ} \mathrm{C}$; UV $(\mathrm{MeOH}) \lambda_{\max }(\varepsilon) 258 \mathrm{~nm}$; IR (KBr) $v_{\max } 3434,3368,1755,1699,1519,1289,1250,1194$, $1172,1102,1074,794,741,723,561 \mathrm{~cm}^{-1} ;{ }^{1} \mathrm{H} \mathrm{NMR}\left(\mathrm{CDCl}_{3}, 300 \mathrm{MHz}\right) \delta 3.97\left(\mathrm{dd}, 1 \mathrm{H},{ }^{2} J=\right.$ $\left.3 \mathrm{~Hz},{ }^{3} J=11 \mathrm{~Hz}, \mathrm{Ser}-\mathrm{H} \beta\right), 4.12\left(\mathrm{dd}, 1 \mathrm{H},{ }^{2} J=3 \mathrm{~Hz},{ }^{3} J=11 \mathrm{~Hz}, \operatorname{Ser}-\mathrm{H} \beta\right), 4.58\left(\mathrm{ddd}, 1 \mathrm{H},{ }^{3} J=4\right.$ $\left.\mathrm{Hz},{ }^{3} J=4 \mathrm{~Hz},{ }^{3} J=11 \mathrm{~Hz}, \mathrm{Ser}-\mathrm{H} \alpha\right), 4.71\left(\mathrm{~d}, 1 \mathrm{H},{ }^{2} J=12 \mathrm{~Hz}, \mathrm{Tce}-\mathrm{CH}_{2}\right), 4.88\left(\mathrm{~d}, 1 \mathrm{H},{ }^{2} J=12\right.$ Hz, Tce-CH $\mathrm{CH}_{2}$, 5.12 (s, br., 2H, Z-CH ), 5.82 (d, 1H, ${ }^{3} \mathrm{~J}=8 \mathrm{~Hz}$, Ser-NH), 7.30-7.36 (m, 5H, Zaromat. $\mathrm{CH}) ;{ }^{13} \mathrm{C} \mathrm{NMR}\left(\mathrm{CDCl}_{3}, 150 \mathrm{MHz}\right) \delta 56.0(\mathrm{Ser}-\mathrm{CH} \alpha), 63.0\left(\mathrm{Ser}-\mathrm{CH}_{2} \beta\right), 67.3\left(\mathrm{Z}-\mathrm{CH}_{2}\right)$, 74.5 (Tce- $\mathrm{CH}_{2}$ ), $94.3\left(\right.$ Tce- $\mathrm{CCl}_{3}$ ), 128.1, 128.3, 128.5 (Z-aromat. CH), 135.9 (Z-quart. C), 156.0 (Z-CO), 169.1 (CO); ESI-MS ( $\left.\mathrm{H}_{2} \mathrm{O} / \mathrm{ACN} / \mathrm{TFA}\right) \quad \mathrm{m} / \mathrm{z} \quad 371 \quad\left([\mathrm{M}+\mathrm{H}]^{+}, \quad 70\right), 412$ $\left([\mathrm{M}+\mathrm{ACN}+\mathrm{H}]^{+}, 100\right)$. 
Z-D-Ser(N-Boc-L-Val)-OTce 6a. ${ }^{12 \mathrm{a}}$ To a solution of Boc-L-Val-OH (7.26 g, $33.4 \mathrm{mmol}$, 1.5 equiv. $)$ in pyridine $(60 \mathrm{~mL})$ at $-10{ }^{\circ} \mathrm{C}$ was added sequentially $\mathrm{HOBt}(4.51 \mathrm{~g}, 33.4 \mathrm{mmol}$, 1.5 equiv.) and a solution of $5 \mathrm{a}(8.25 \mathrm{~g}, 22.3 \mathrm{mmol})$ in $20 \mathrm{~mL}$ pyridine. After stirring for 5 min, the mixture was treated with DCC (6.89 g, $33.4 \mathrm{mmol}, 1.5$ equiv.) in pyridine $(20 \mathrm{~mL})$ and stirred for $18 \mathrm{~h}$ at $0{ }^{\circ} \mathrm{C}$. The separated DCU was removed by filtration and the solvent evaporated in vacuo. The oily residue was diluted with ethyl acetate $(150 \mathrm{~mL})$ and washed consecutively with cold $1 \mathrm{~N}$ aqueous $\mathrm{HCl}(150 \mathrm{~mL}), 5 \%$ aqueous $\mathrm{NaHCO}_{3}(150 \mathrm{~mL})$, and saturated aqueous $\mathrm{NaCl}(100 \mathrm{~mL})$. The solution was dried $\left(\mathrm{MgSO}_{4}\right)$, filtered and concentrated in vacuo. 6a (11.2 $\mathrm{g}, 19.6 \mathrm{mmol}, 88 \%)$ was obtained after purification by flash chromatography $\left(\mathrm{SiO}_{2}, 6 \times 15 \mathrm{~cm}, 33 \%\right.$ ethyl acetate-hexane) as a colorless oil: TLC (35\% ethyl acetate/hexane) $R_{f} 0.31 ;[\mathrm{a}]^{25}{ }_{\mathrm{D}}+4(\mathrm{c} 0.05, \mathrm{MeOH}) ; \mathrm{UV}(\mathrm{MeOH}) \lambda_{\max }(\varepsilon) 258 \mathrm{~nm}$; IR $(\mathrm{KBr}) v_{\max } 3442,2975,1720,1527,1369,1340,1274,1196,1150,1090,1060,569 \mathrm{~cm}^{-1} ;{ }^{1} \mathrm{H}$ $\operatorname{NMR}\left(\mathrm{CDCl}_{3}, 300 \mathrm{MHz}\right) \delta 0.83\left(\mathrm{~d}, 3 \mathrm{H},{ }^{3} \mathrm{~J}=7 \mathrm{~Hz}, \mathrm{Val}-\mathrm{CH}_{3}\right), 0.90\left(\mathrm{~d}, 3 \mathrm{H},{ }^{3} \mathrm{~J}=7 \mathrm{~Hz}\right.$, Val$\left.\mathrm{CH}_{3}\right), 1.37\left(\mathrm{~s}, 9 \mathrm{H}, \mathrm{Bu}-\mathrm{CH}_{3}\right), 1.94-2.05(\mathrm{~m}, 1 \mathrm{H}, \mathrm{Val}-\mathrm{H} \beta), 4.06\left(\mathrm{dd}, 1 \mathrm{H},{ }^{3} \mathrm{~J}=5 \mathrm{~Hz},{ }^{3} \mathrm{~J}=8 \mathrm{~Hz}\right.$,

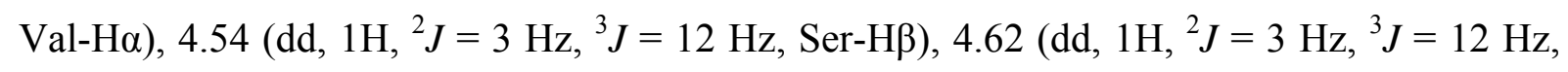

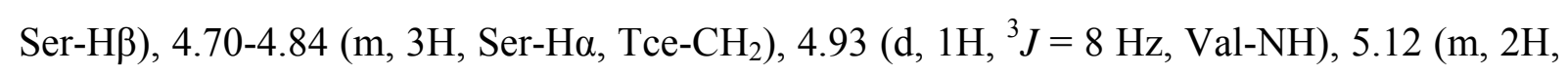
Z-CH 2$), 5.92\left(\mathrm{~d}, 1 \mathrm{H},{ }^{3} \mathrm{~J}=8 \mathrm{~Hz}\right.$, Ser-NH), 7.28-7.34 (m, 5H, Z-aromat. $\left.\mathrm{CH}\right) ;{ }^{13} \mathrm{C}$ NMR $\left(\mathrm{CDCl}_{3}, 150 \mathrm{MHz}\right) \delta 17.7,19.0\left(\mathrm{Val}-\mathrm{CH}_{3}\right), 28.2\left(t \mathrm{Bu}-\mathrm{CH}_{3}\right), 30.7$ (Val-CH $\left.\beta\right), 53.4(\mathrm{Ser}-\mathrm{CH} \alpha)$, $58.9($ Val-CH $\alpha), 64.2\left(\right.$ Ser- $\left.\mathrm{CH}_{2} \beta\right), 67.3\left(\mathrm{Z}-\mathrm{CH}_{2}\right), 74.8\left(\right.$ Tce- $\left.\mathrm{CH}_{2}\right), 80.2$ ( $t$ Bu-quart. C), 94.2 (Tce-CCl ${ }_{3}$ ), 128.2, 128.5 (Z-aromat. CH), 135.9 (Z-quart. C), 155.6, 156.0 (Boc-CO, Z-CO), 168.0, 172.1 (Ser-CO, Val-CO); ESI-MS (MeOH) m/z $591\left([\mathrm{M}+\mathrm{Na}]^{+}, 100\right)$.

\section{(Z-D-Ser-L-Ala-L-Cys-L-Val) ${ }_{2}$ (serine hydroxyl) dilactone disulfide $14 a^{12 b-d}$}

TLC (ethyl acetate) $R_{f} 0.47 ;{ }^{1} \mathrm{H}$ NMR (DMSO- $\left.d_{6}, 600 \mathrm{MHz}\right) \delta 0.859\left(\mathrm{~d}, 6 \mathrm{H},{ }^{3} J=7 \mathrm{~Hz}, \mathrm{Val}-\right.$ $\left.\mathrm{CH}_{3}\right), 0.864\left(\mathrm{~d}, 6 \mathrm{H},{ }^{3} \mathrm{~J}=7 \mathrm{~Hz}, \mathrm{Val}-\mathrm{CH}_{3}\right), 1.24\left(\mathrm{~d}, 6 \mathrm{H},{ }^{3} \mathrm{~J}=7 \mathrm{~Hz}\right.$, Ala- $\left.\mathrm{CH}_{3}\right), 2.18-2.24(\mathrm{~m}, 2 \mathrm{H}$, 


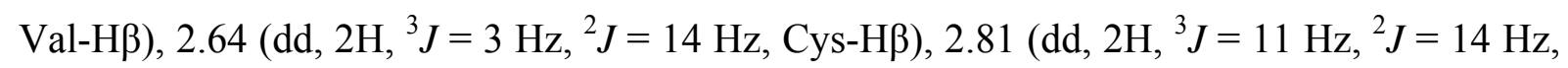
Cys-H $\beta$ ), $4.11\left(\mathrm{dd}, 2 \mathrm{H},{ }^{3} \mathrm{~J}=3 \mathrm{~Hz},{ }^{2} J=11 \mathrm{~Hz}, \operatorname{Ser}-\mathrm{H} \beta\right), 4.20\left(\mathrm{dd}, 2 \mathrm{H},{ }^{3} \mathrm{~J}=10 \mathrm{~Hz},{ }^{3} \mathrm{~J}=10 \mathrm{~Hz}\right.$,

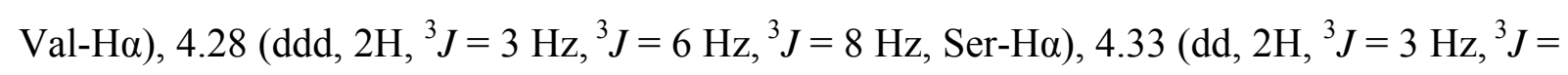
$11 \mathrm{~Hz}, \mathrm{Ser}-\mathrm{H} \beta), 4.38$ (dq, 2H, ${ }^{3} J=7 \mathrm{~Hz},{ }^{3} J=7 \mathrm{~Hz}, \mathrm{Ala}-\mathrm{H} \alpha$ ), 5.05-5.11 (m, br., 4H, Z-CH 2 , 5.24 (ddd, $2 \mathrm{H},{ }^{3} \mathrm{~J}=5 \mathrm{~Hz},{ }^{3} \mathrm{~J}=10 \mathrm{~Hz},{ }^{3} \mathrm{~J}=10 \mathrm{~Hz}, \mathrm{Cys}-\mathrm{H \alpha}$ ), 7.31-7.37 (m, 10H, Z-aromat. CH), 7.52-7.57 (m, br., 2H, Ser-NH), 7.74 (d, 2H, ${ }^{3} J=7 \mathrm{~Hz}$, Ala-NH), 8.16 (d, 2H, ${ }^{3} J=10 \mathrm{~Hz}$, Val-NH), $8.66\left(\mathrm{~d}, 2 \mathrm{H},{ }^{3} \mathrm{~J}=10 \mathrm{~Hz}, \mathrm{Cys}-\mathrm{NH}\right) ;{ }^{13} \mathrm{C}$ NMR (DMSO- $\left.d_{6}, 150 \mathrm{MHz}\right) \delta 18.1$ (Ala$\left.\mathrm{CH}_{3}\right), 18.2\left(\right.$ Val- $\left.\mathrm{CH}_{3}\right), 18.9\left(\right.$ Val- $\left.\mathrm{CH}_{3}\right), 30.1$ (Val-CH $\left.\beta\right), 44.1\left(\mathrm{Cys}_{-} \mathrm{CH}_{2} \beta\right), 48.0$ (Ala-CH $\left.\alpha\right)$,

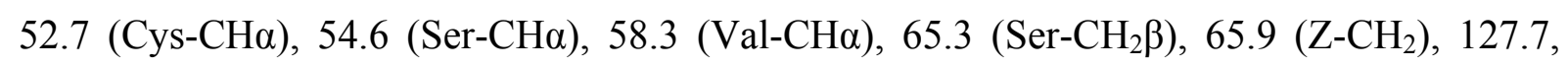
127.8, 128.3 (Z-aromat. CH), 136.6 (Z-quart. C), 155.6 (Z-CO), 167.8, 169.3, 169.5, 173.1 (Ser-CO, Val-CO, Cys-CO, Ala-CO); ESI-MS (MeOH) m/z $1009\left([\mathrm{M}+\mathrm{Na}]^{+}, 100\right), 1995$ $\left([2 \mathrm{M}+\mathrm{Na}]^{+}, 40\right)$. 


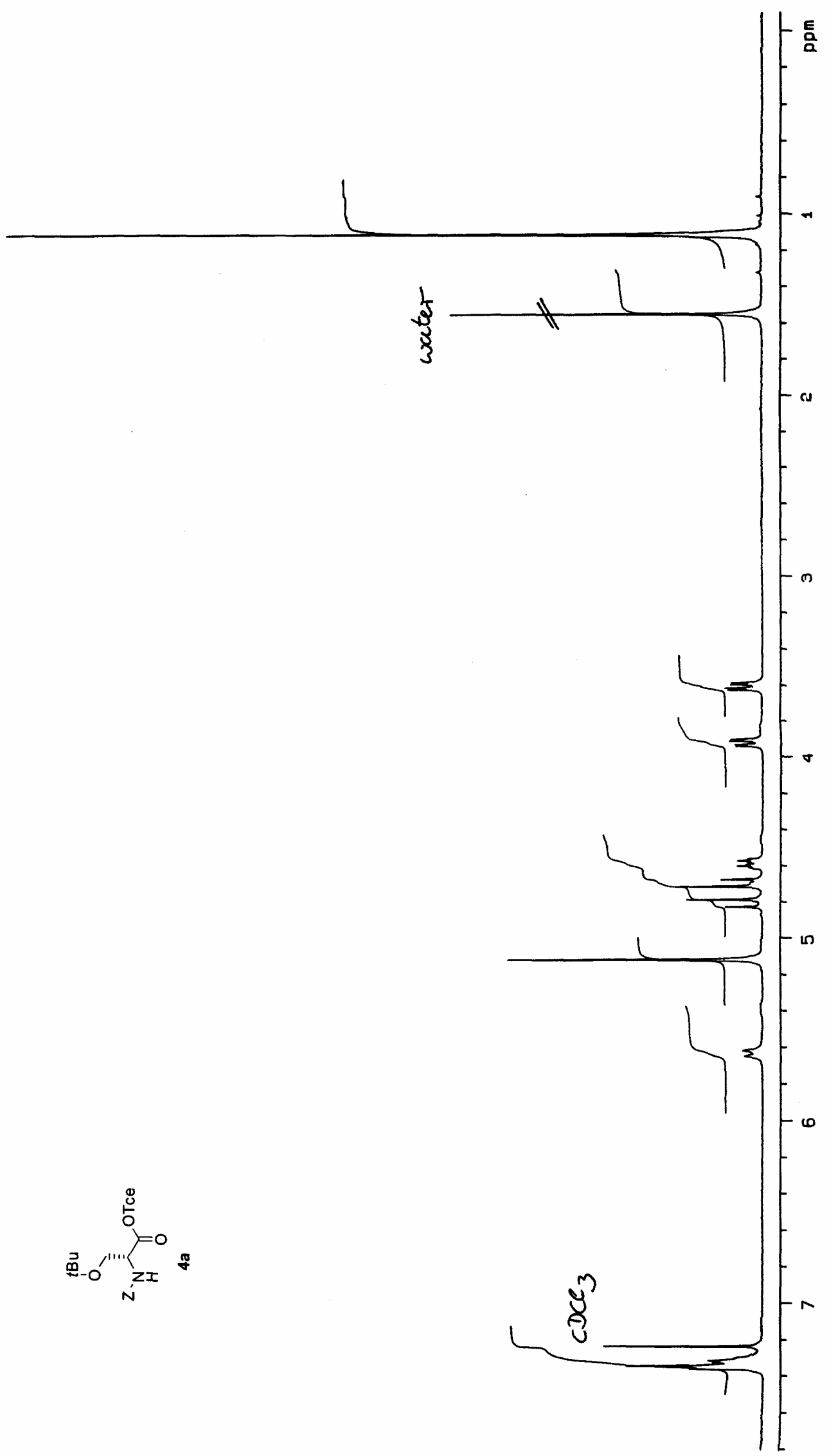


$909 \cdot 19$

$\angle \varepsilon Z^{\prime} \angle 9$

$9 \varepsilon 9^{\circ} \varepsilon L$

$\angle \angle 9^{\circ} \triangleright L$

เレย๋6

291.821

$0 \angle 9.821 \longrightarrow$

๕२०'9६เ

$89 l \cdot 99 l$

$6 เ+69 t$
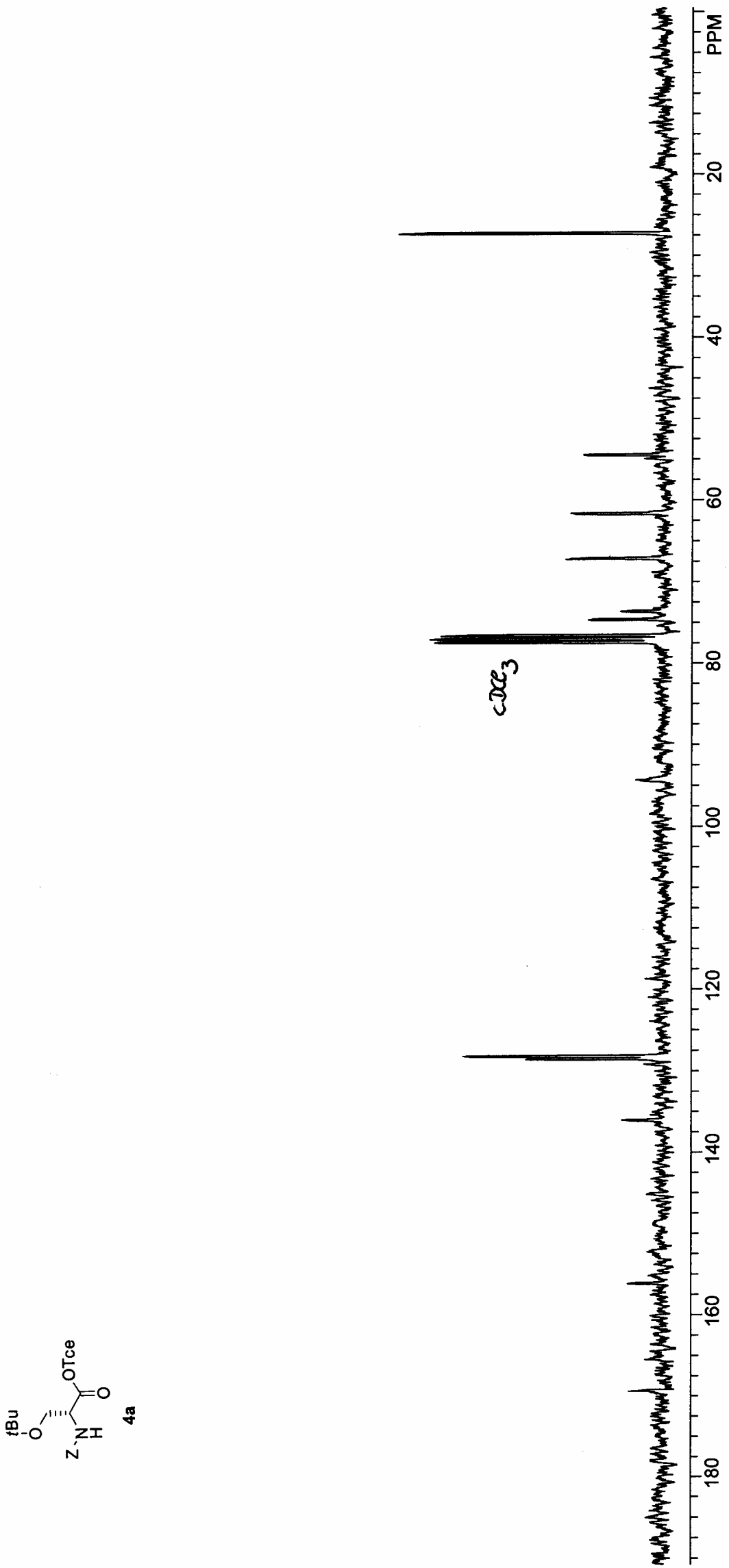
260 '

TOL' 5

OEl'

工
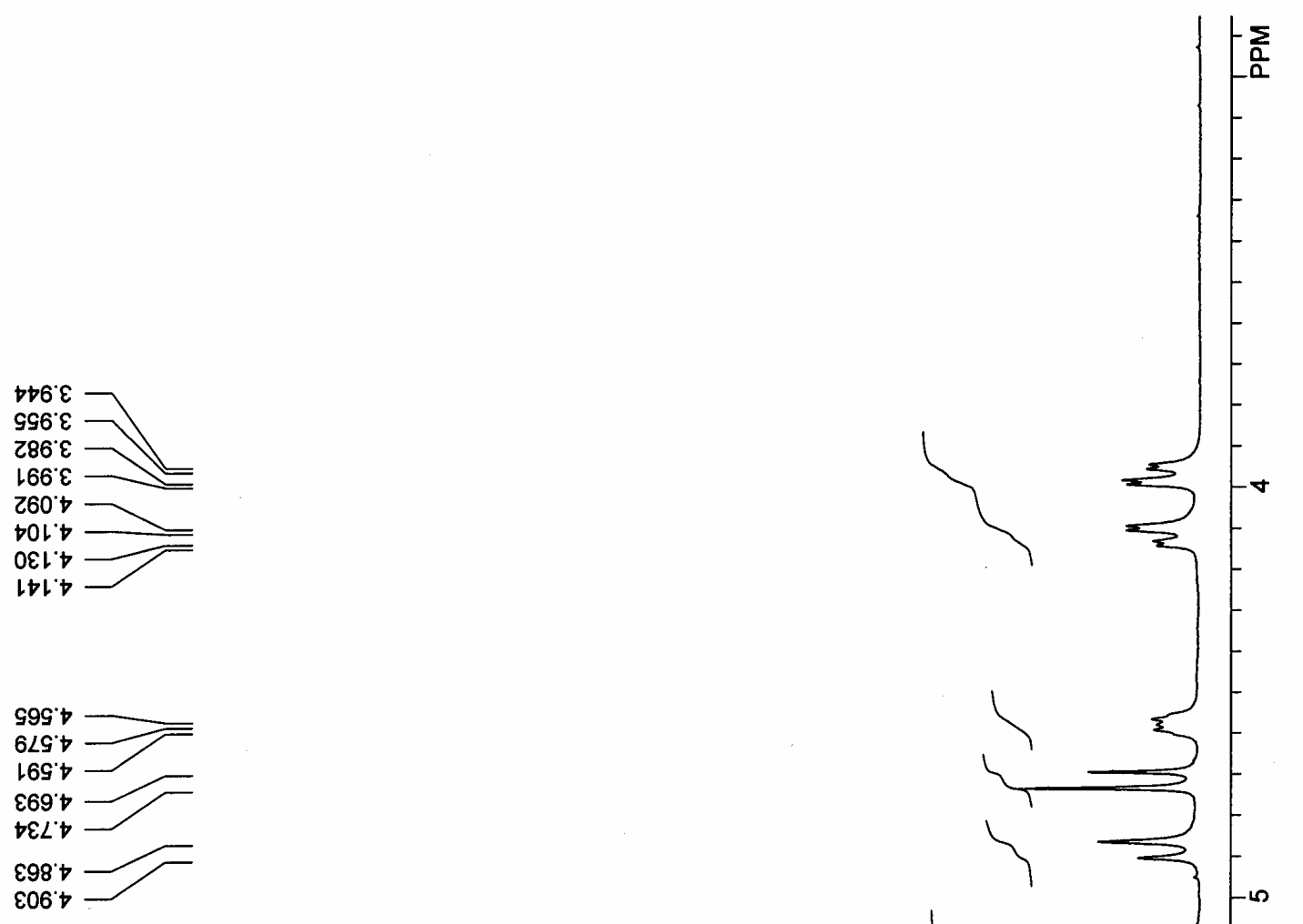

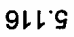

218.9

$\angle 8^{\circ} \mathrm{S}$
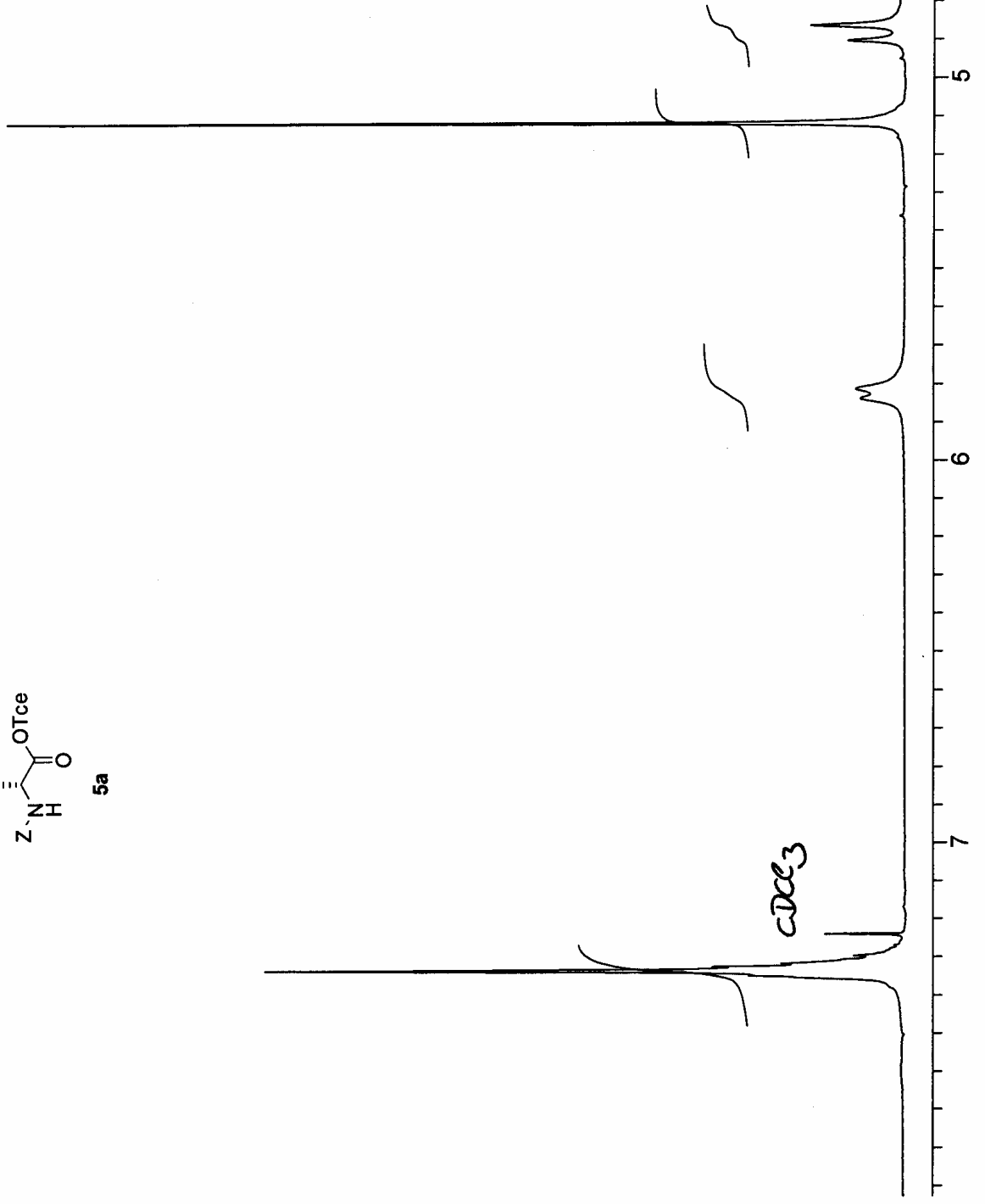

GZE: $L$

$9 \varepsilon \varepsilon \varepsilon^{\prime} L$

$8 \nabla \varepsilon^{\circ} L$<smiles>COC(=O)C(CO)C(=O)O</smiles> 


$$
1
$$




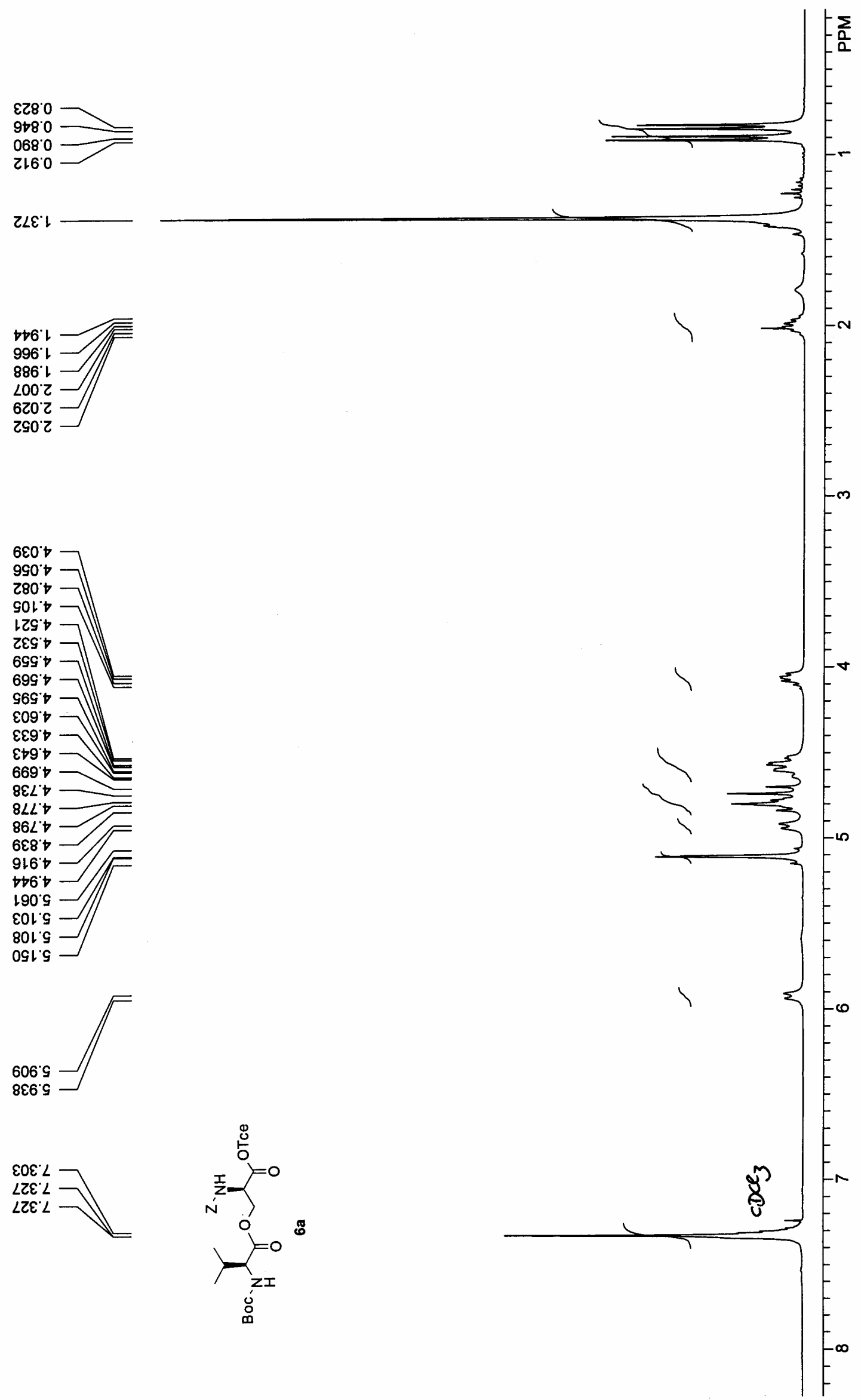


乙๖๘૬

$898^{\circ} 89$

LSL.t9

$6 Z \varepsilon^{\circ} 2$

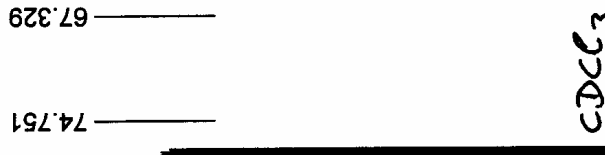

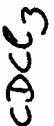

00208

$99 l^{\circ}+6$

602 ' 82

SOS' 82

068 $9 \varepsilon \downarrow$

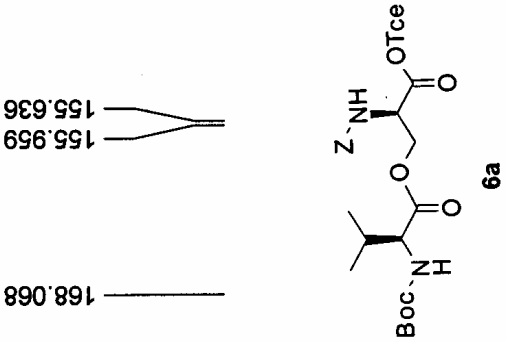

$9 \varepsilon เ \cdot Z<\downarrow$

○.

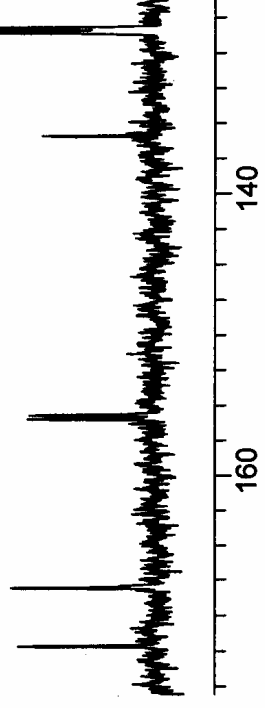




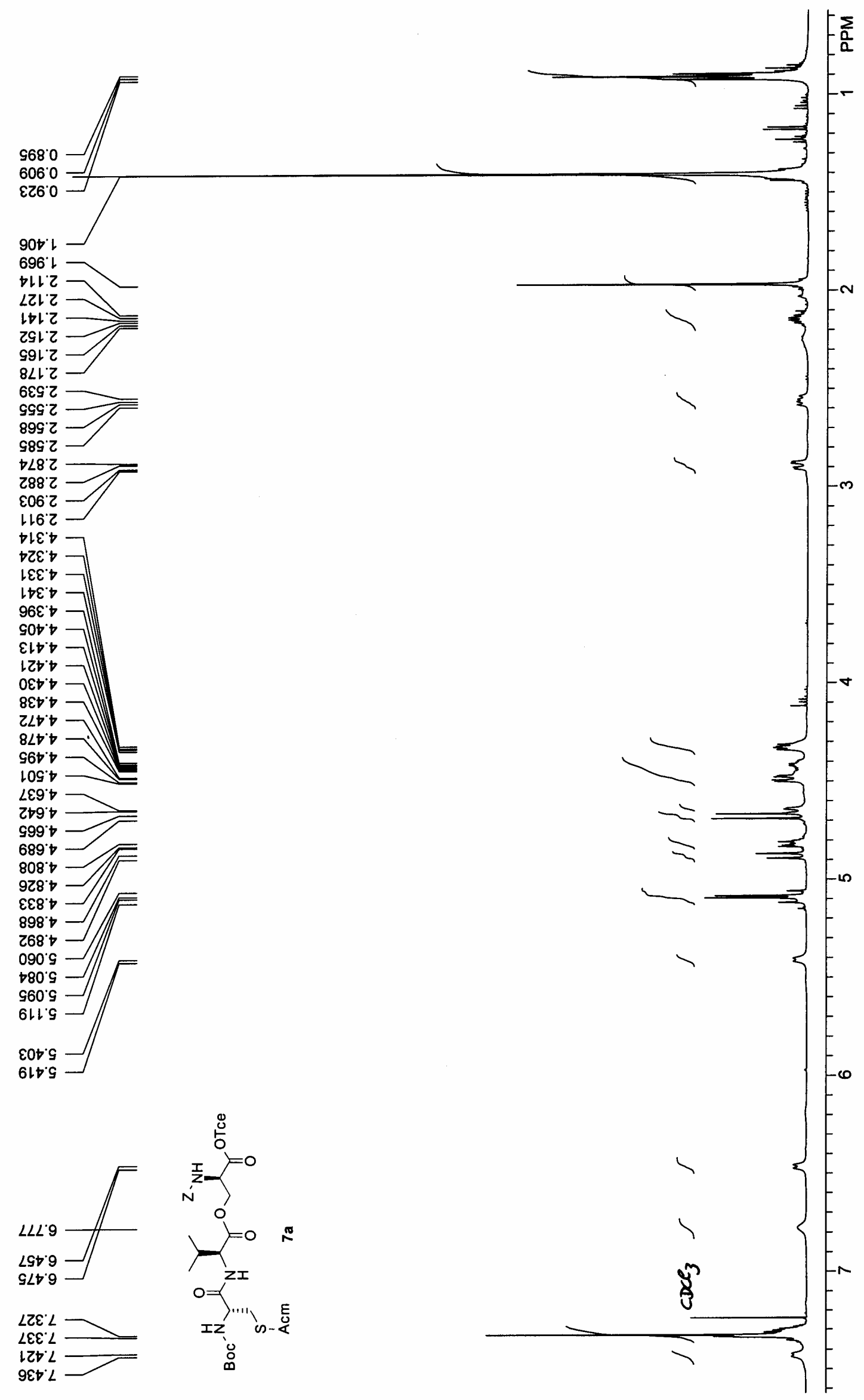


$0 \varepsilon 9 . \angle$

$\angle \varepsilon 6.81$

$\angle 80$ \&乙

$602 \cdot 82$

$1 \angle E^{\circ} 0 \varepsilon$

$896^{\circ} \downarrow \varepsilon$

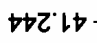

$\varepsilon เ{ }^{\prime} \varepsilon \varsigma$

$128<9$

$28 \varepsilon^{\circ}+9$

$2 L 2 \angle 9-$
$\square 6 S^{\circ} \bullet L-$

$\stackrel{m}{y}$

$9<0.08$
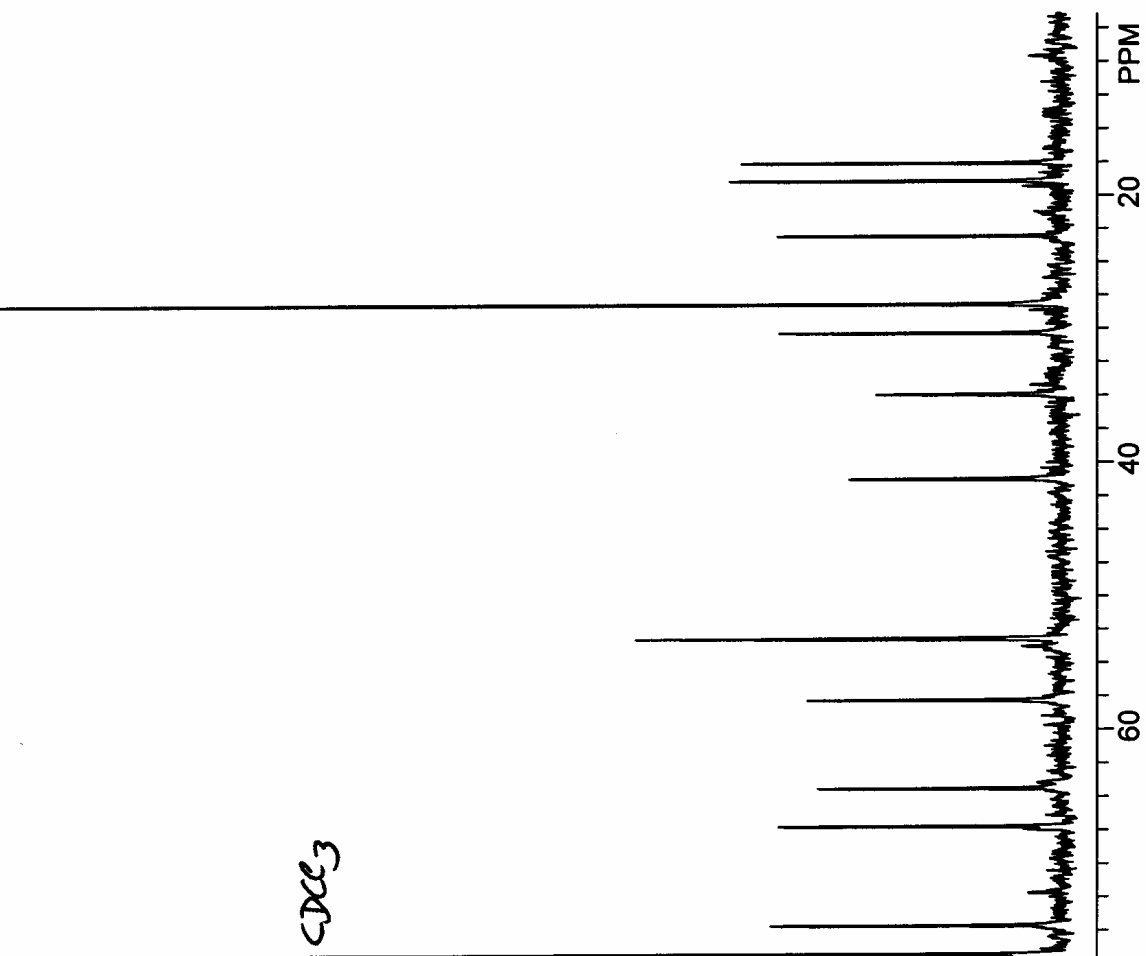

¿งเ"ซ6

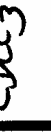

8Eเ ' 821

96l' $82 \mathrm{~L}$

$\varepsilon \angle t 8 Z 1$ -

เZ6 ' $\& \&$
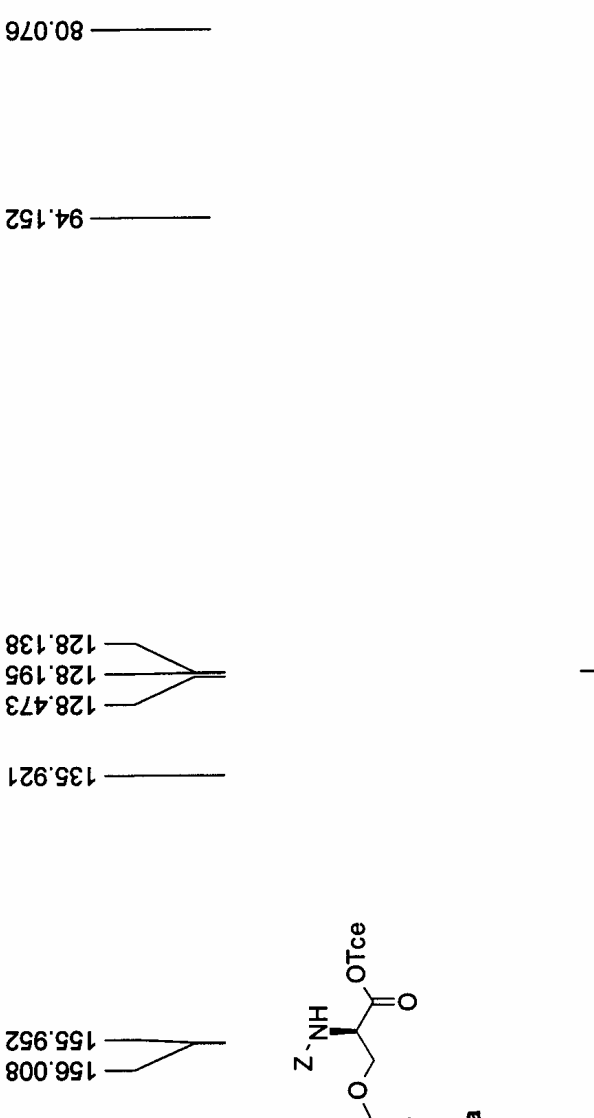

N

I2L.89l816021

ZเTレLL

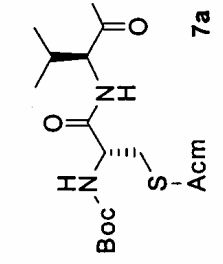

$N$

ह

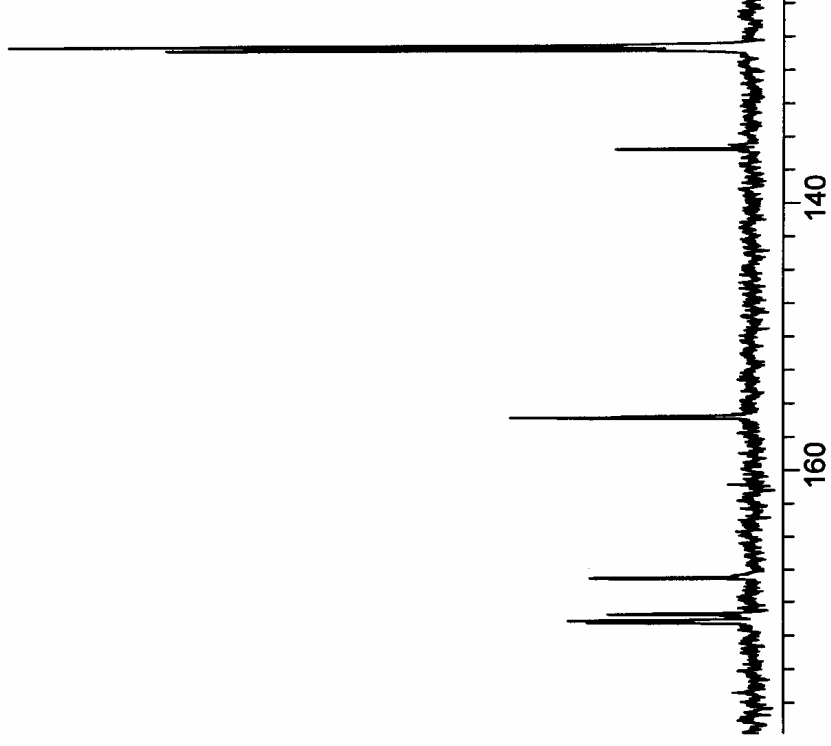




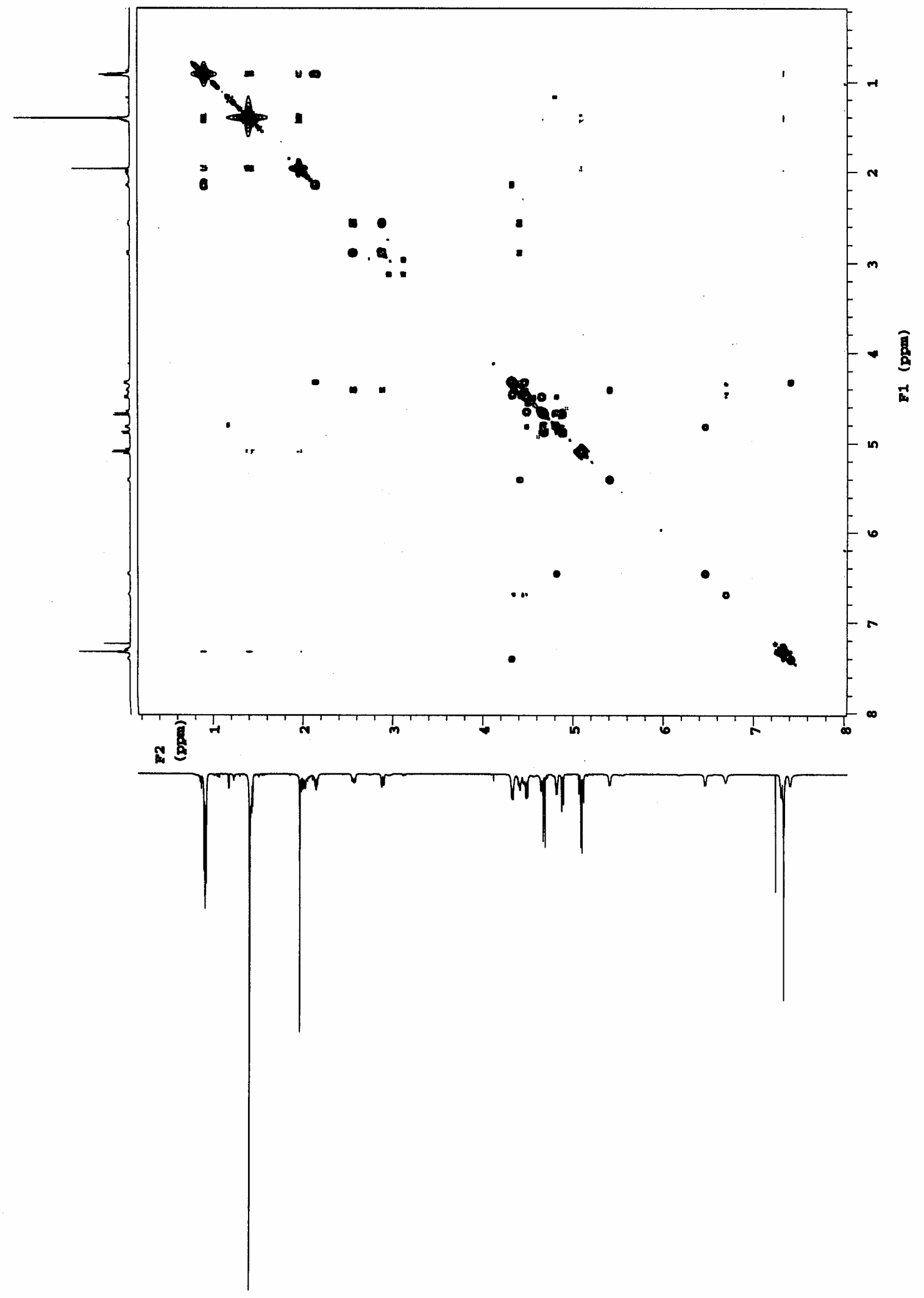




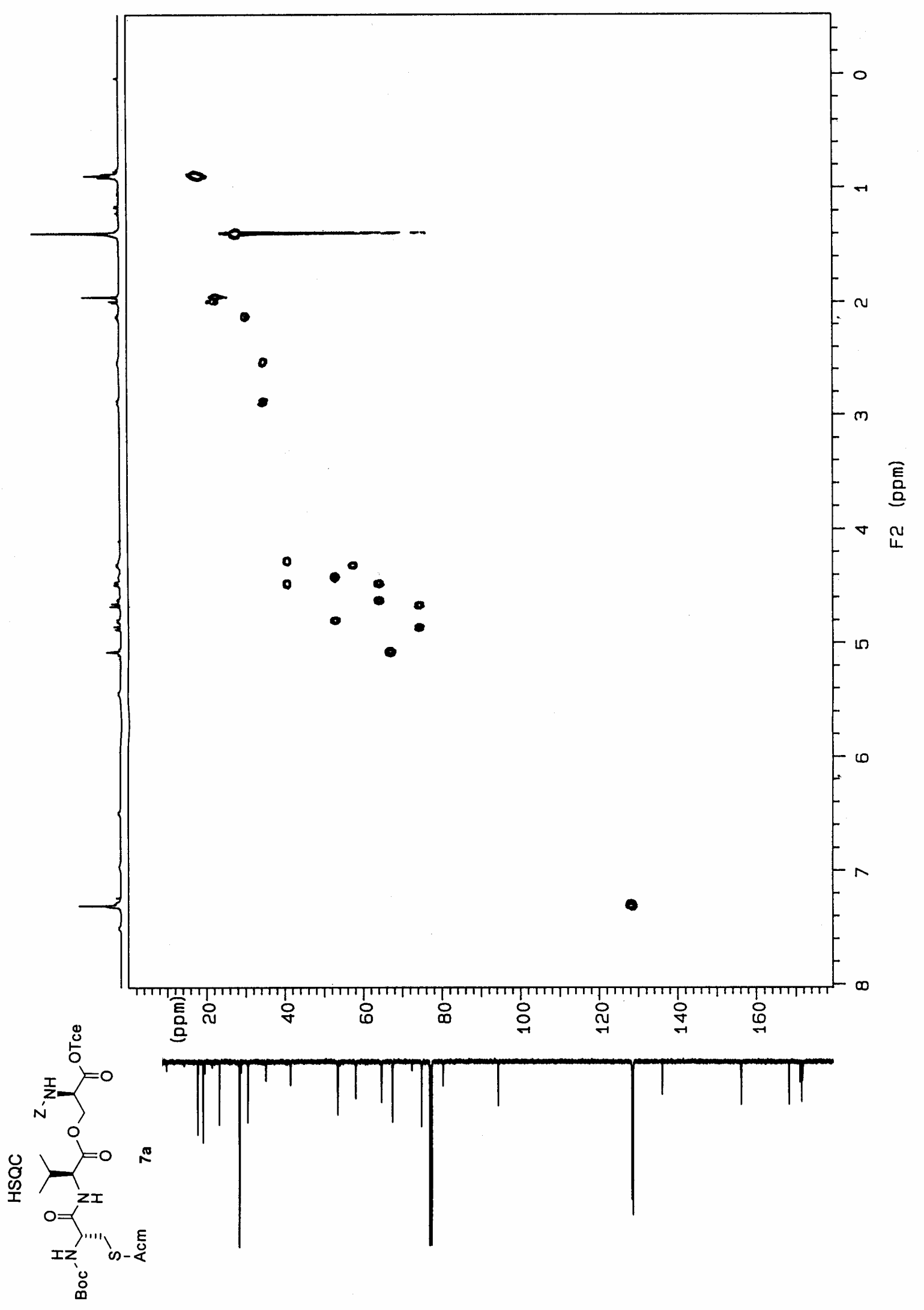




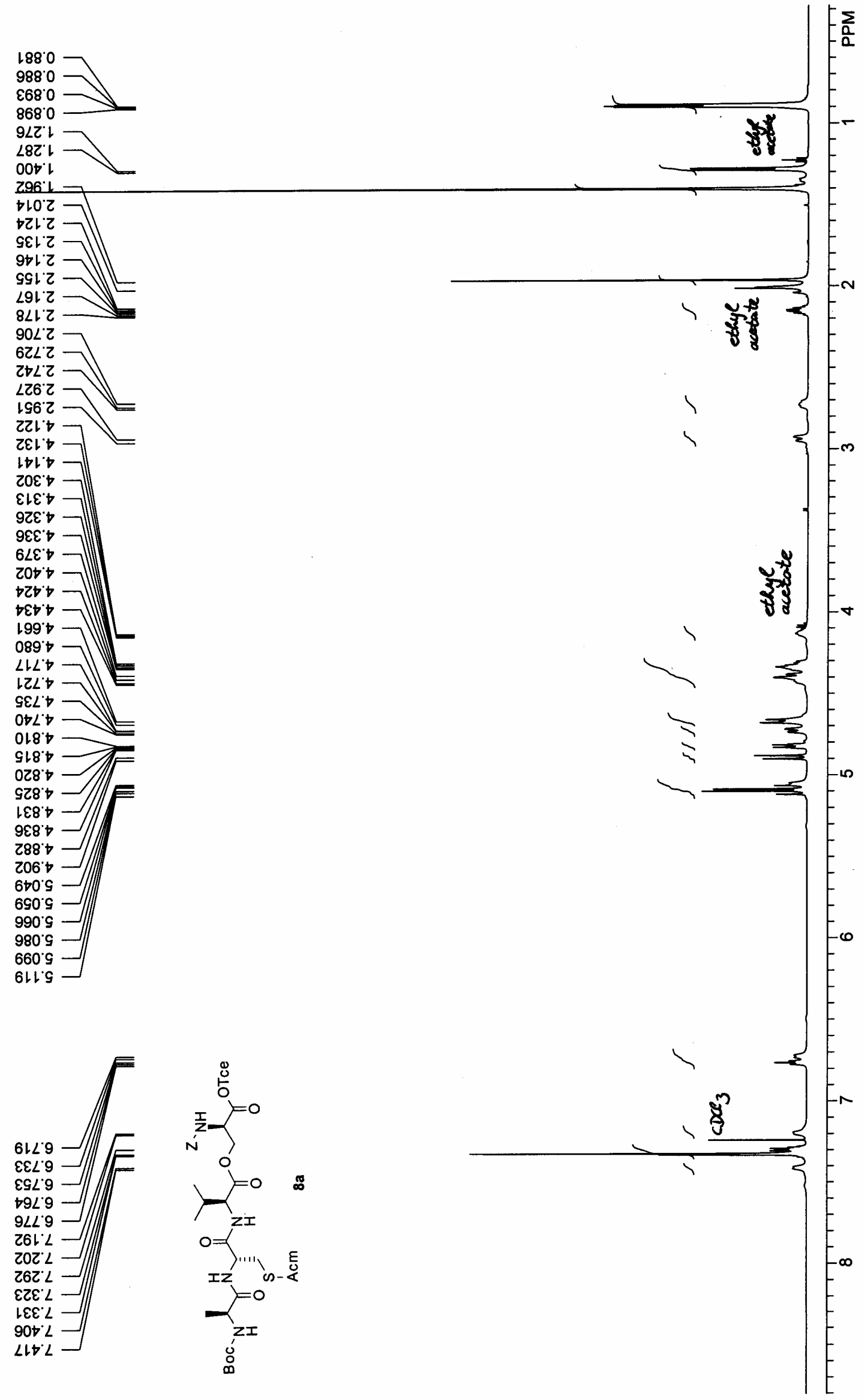


St9. 2

9028

926.81

๕૬乙 ६乙

$6 ৮ 乙 8$

$\succ 89^{\circ} 0 \varepsilon$

$\llcorner\llcorner 6 \varepsilon \varepsilon$

เEG'เ

Sttos

ZLS'ZS

OSZ \&૬ -

$8 Z 8 \angle 9$

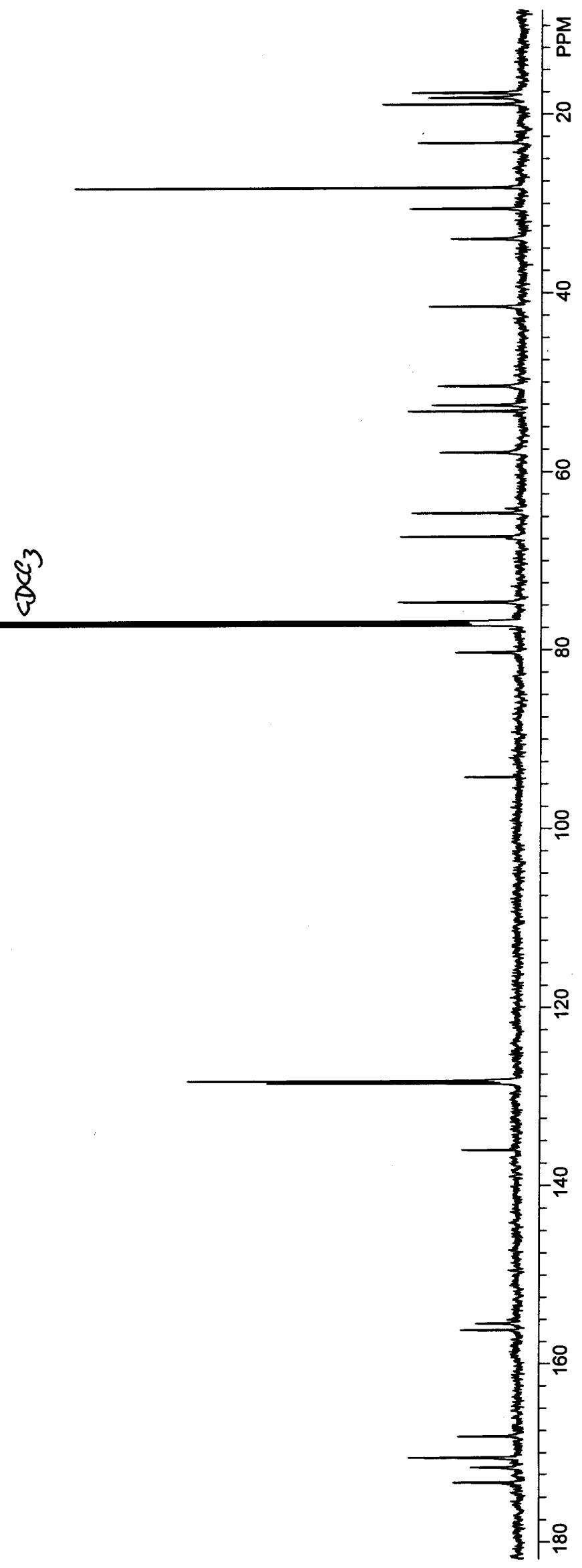

LOL'82L

292 '8ZL

हZS'8Z1 -

$610.9 \varepsilon 1$

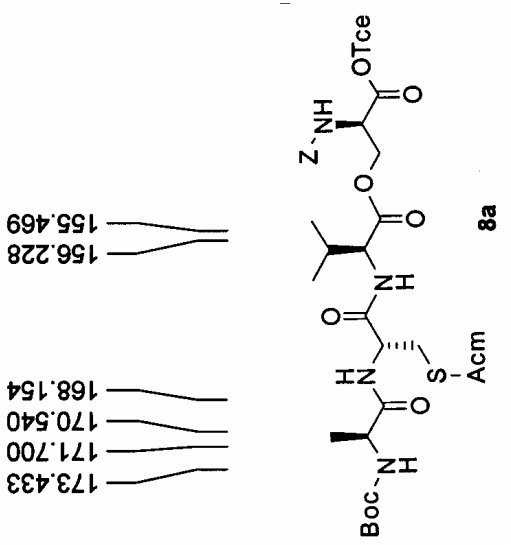

$189^{\circ}+9$

$\downarrow \varepsilon Z \angle 9-$

$\varepsilon \varepsilon 9^{\circ} \downarrow L$

$\angle 9 Z 08$

ZIZ゙ฑ6 


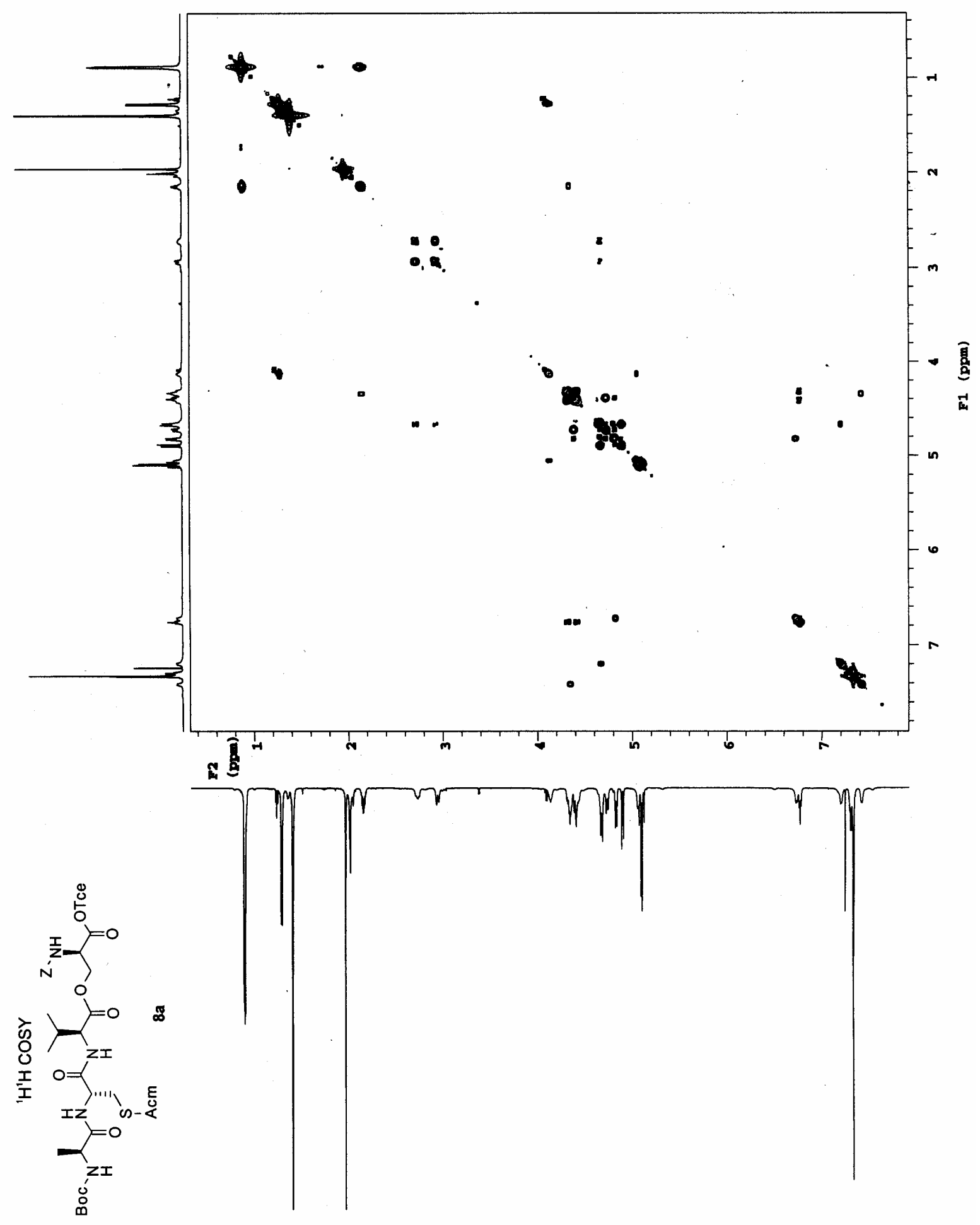




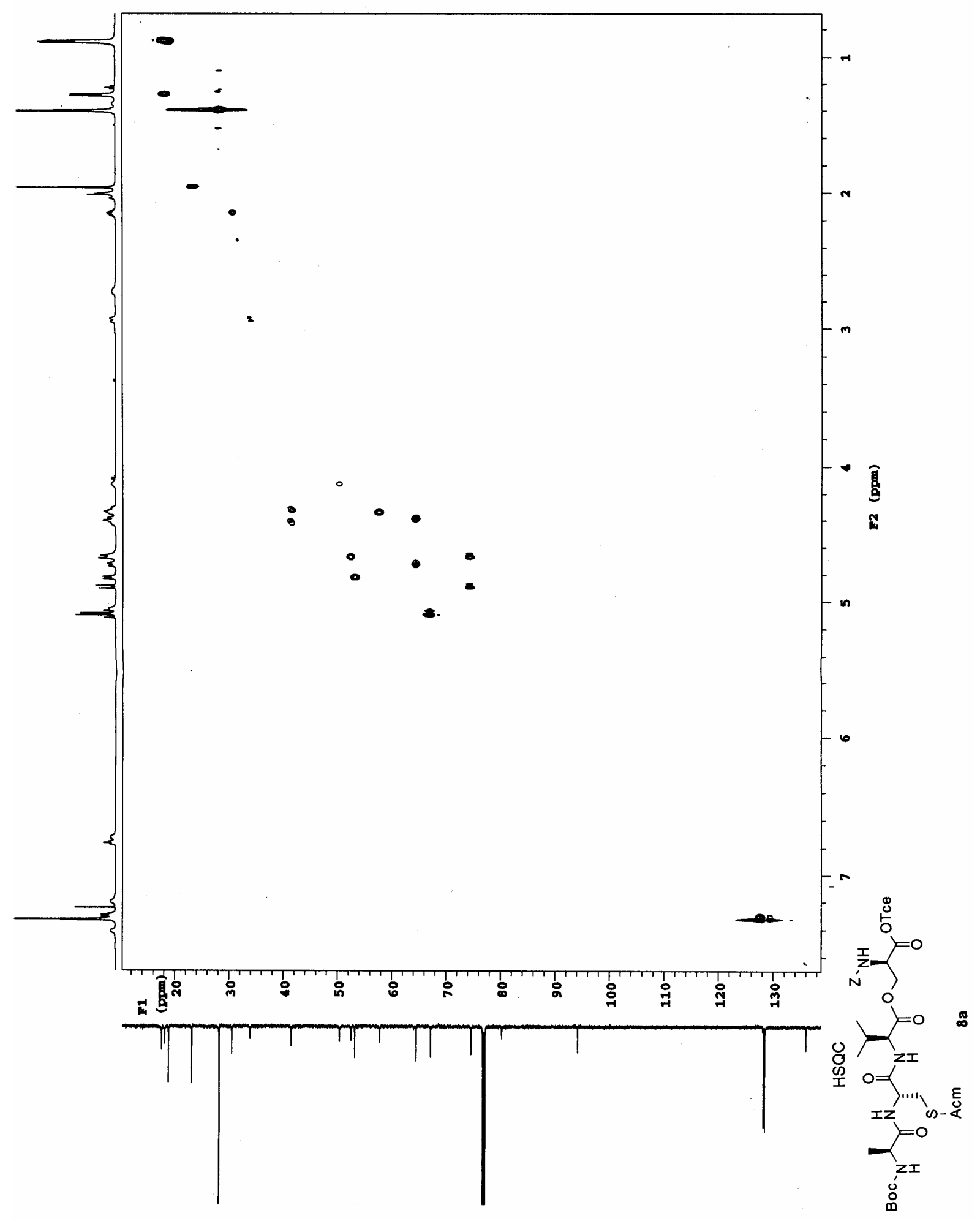




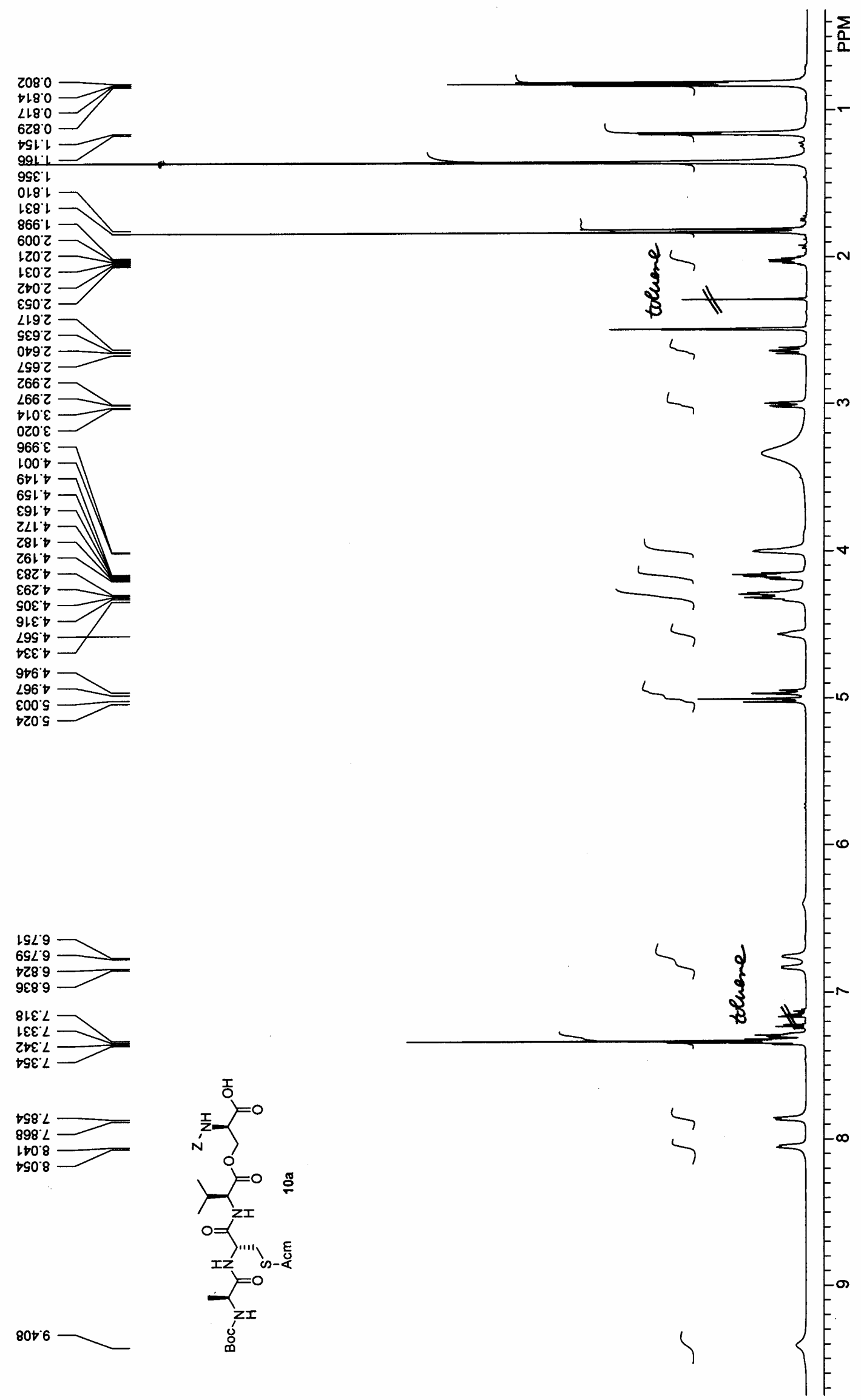



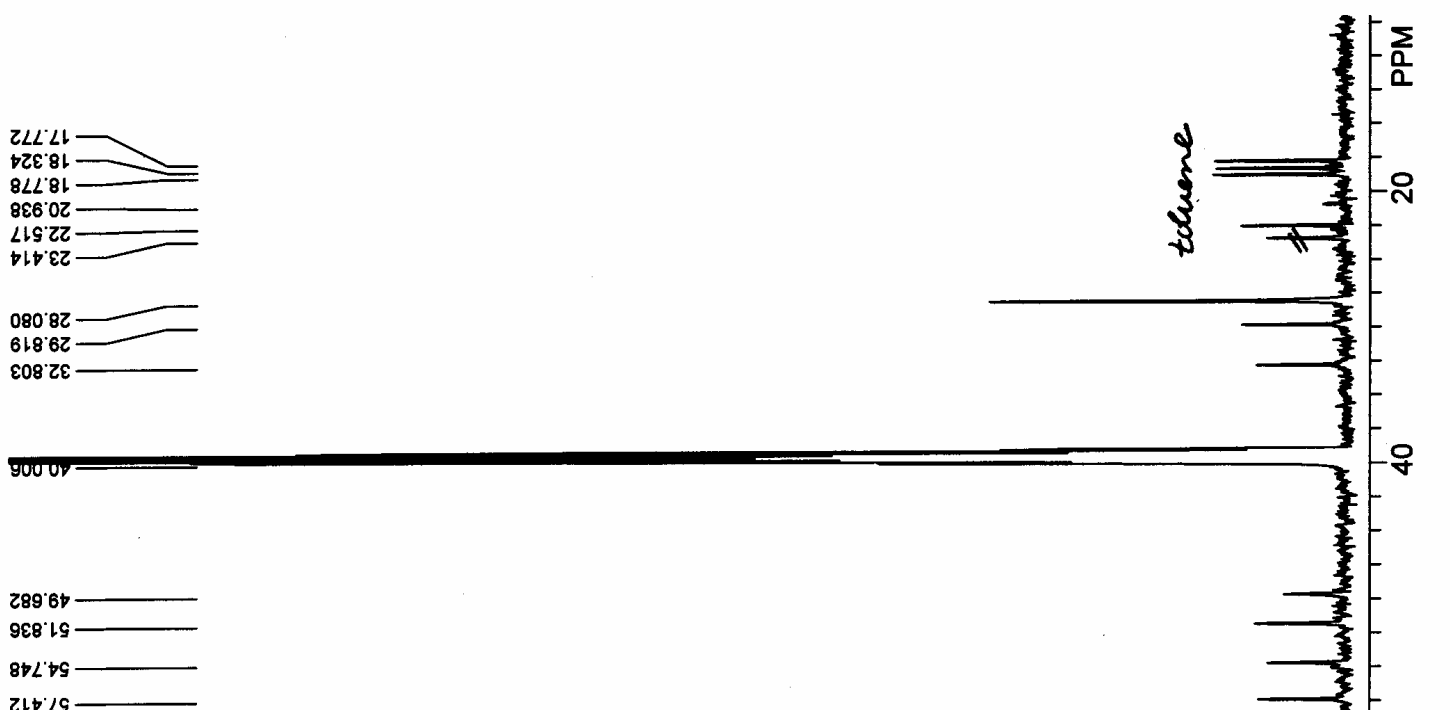

061.99
$819.99-\square$

710.82

$96 S^{\circ} \angle Z L$

$\checkmark S 0.8 Z 1-$

9Lᄂ 8 Z

$\downarrow Z 0^{\circ}\llcorner\varepsilon \downarrow-$
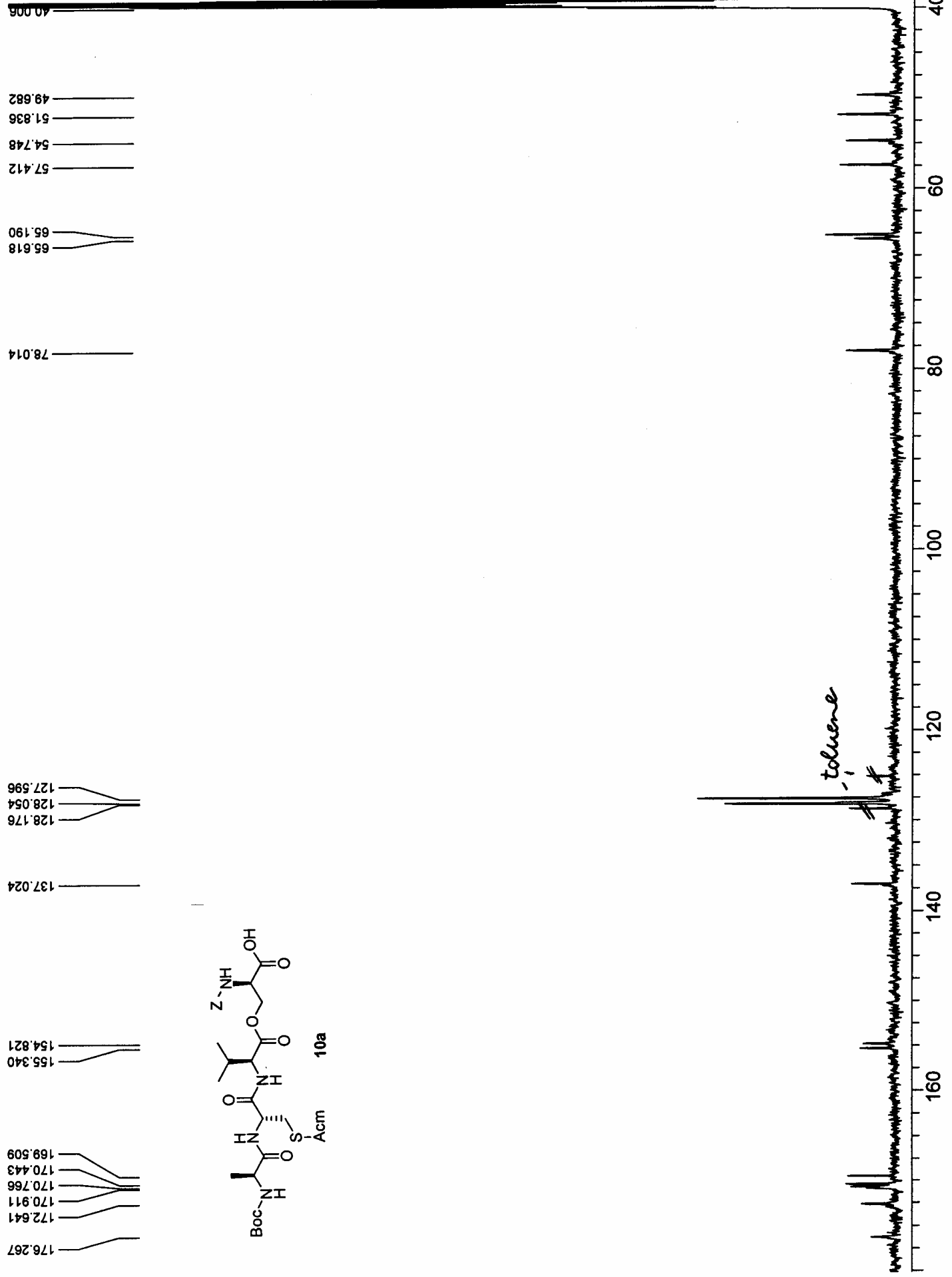


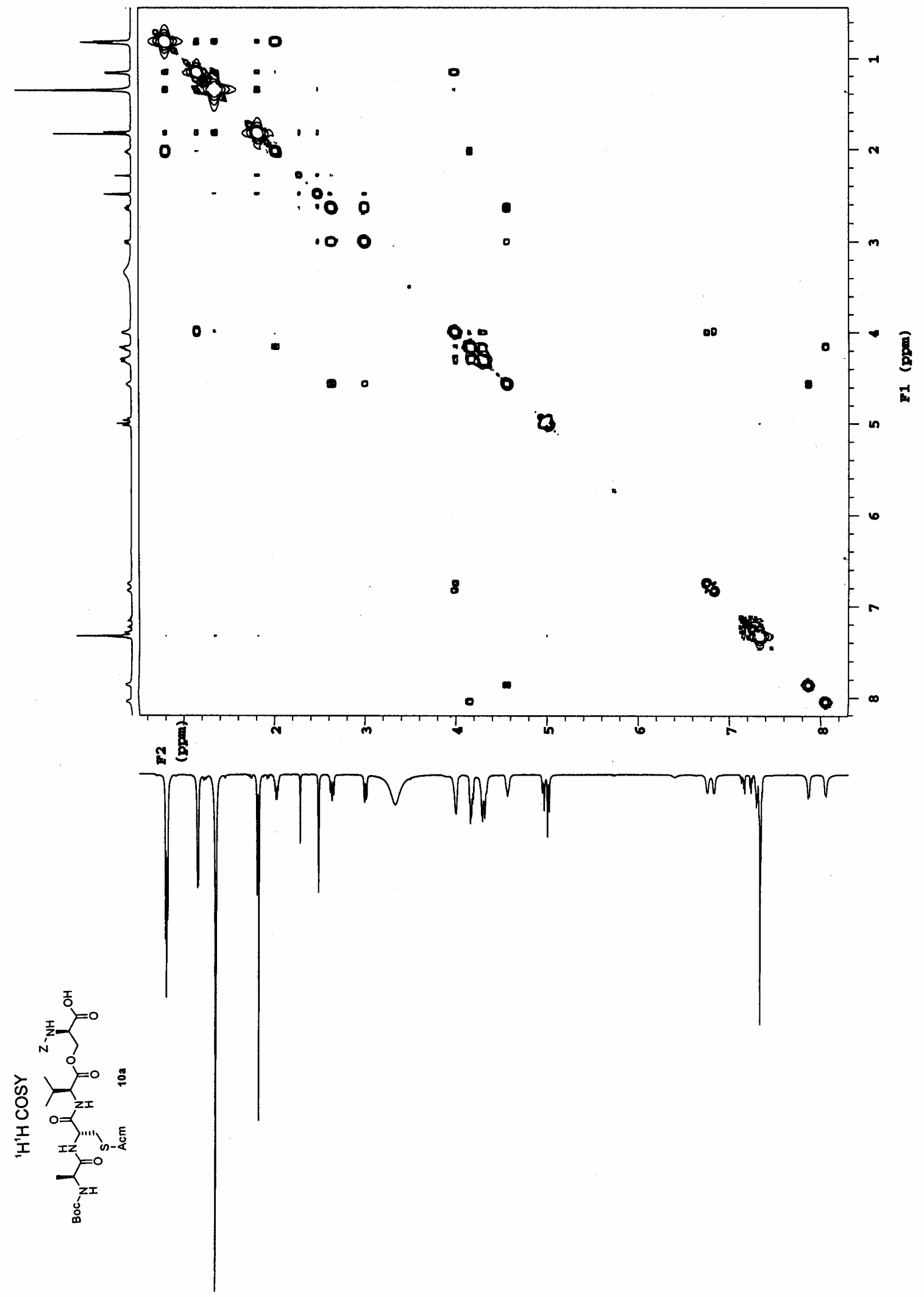




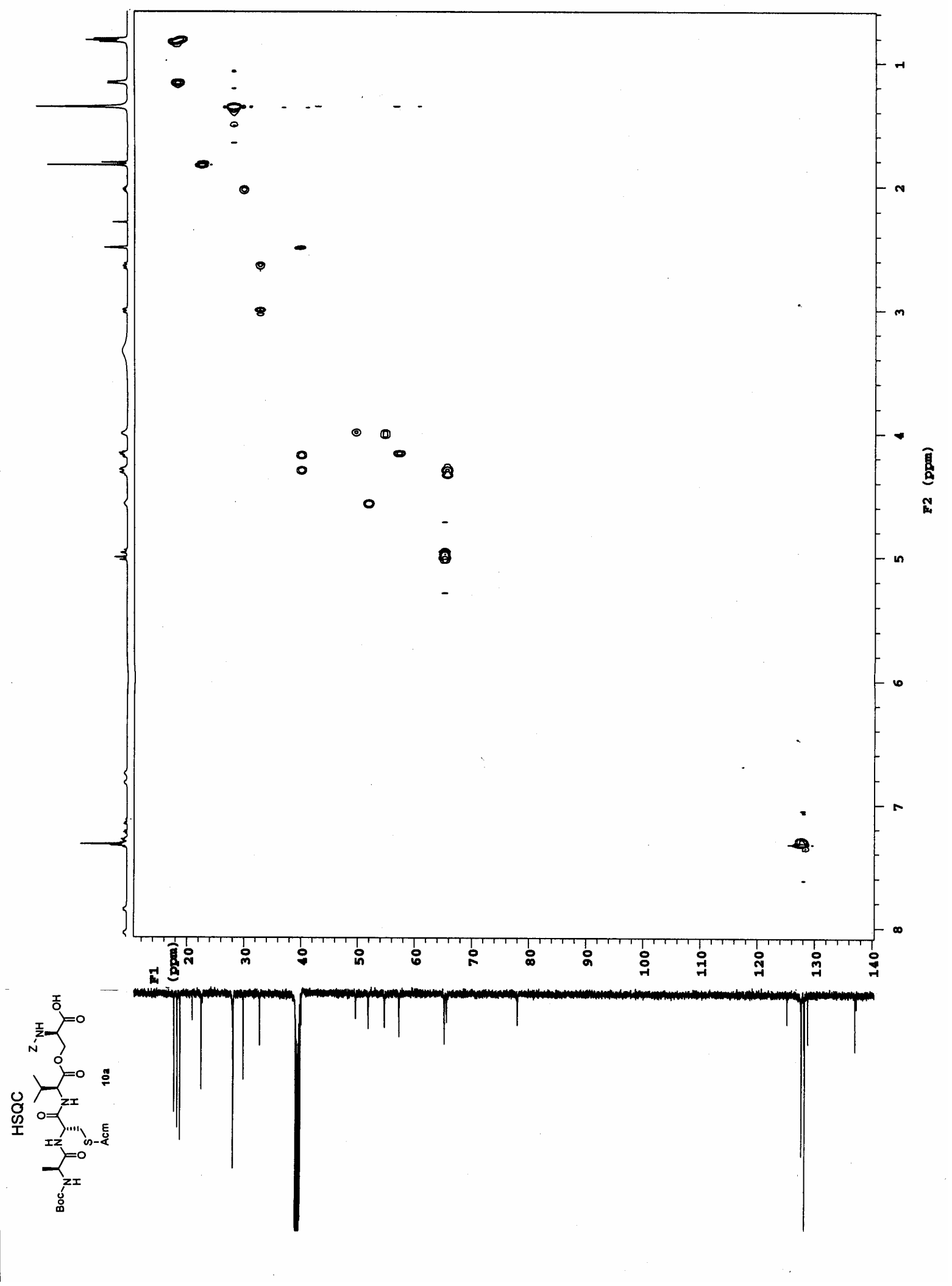



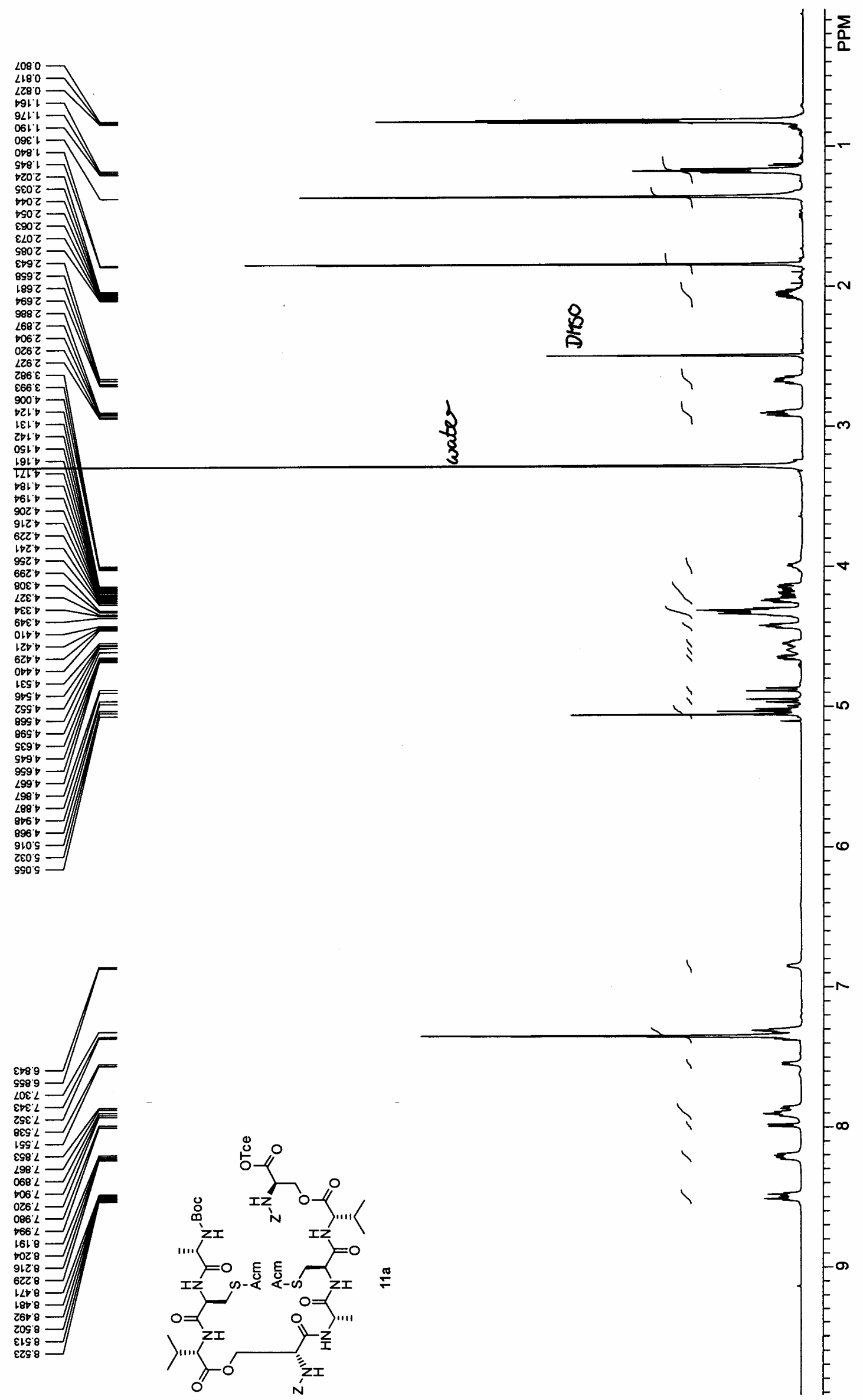

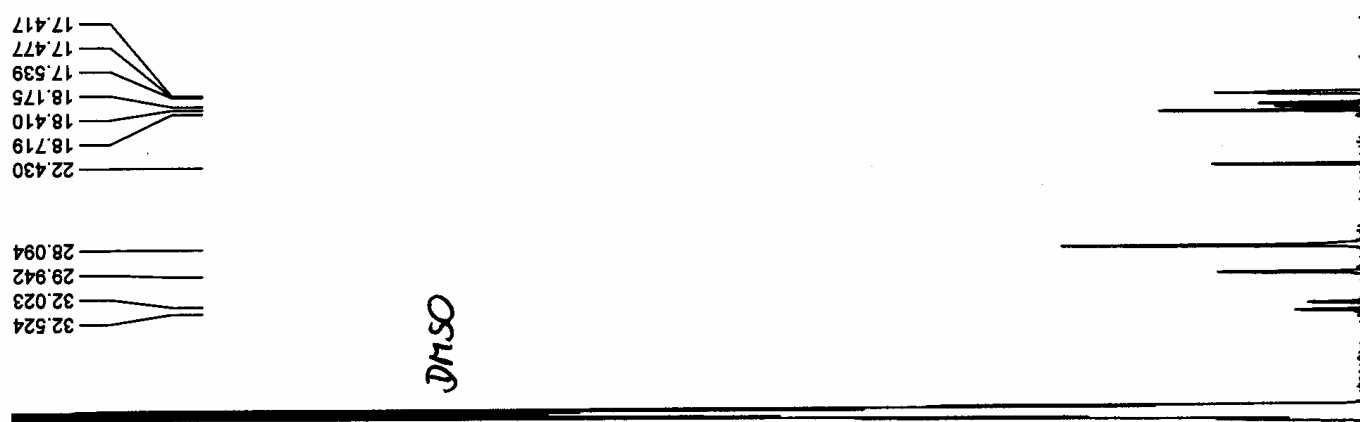

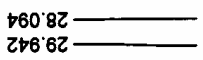

¿Z००८

$\rightarrow Z s \cdot z \varepsilon$

8
$\frac{5}{9}$

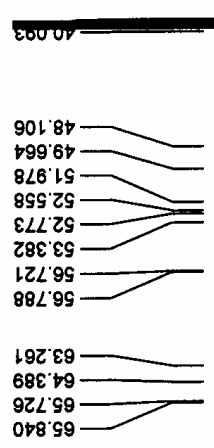

$9 \varepsilon L\llcorner L$

$220 \% 8 L$

$90<\div 6$

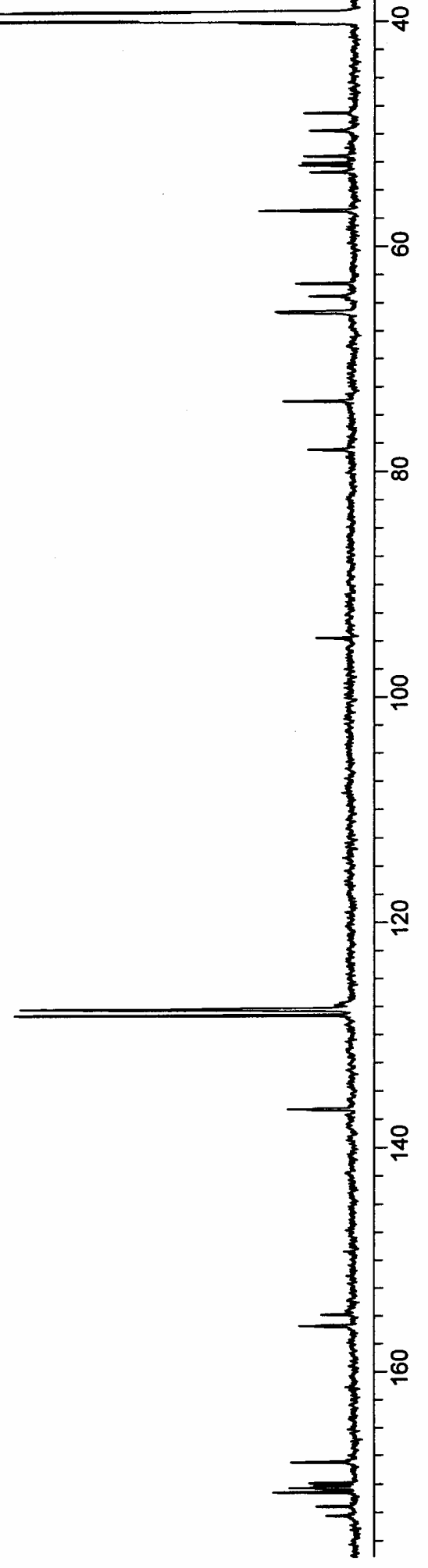

$\rightarrow 8 \angle L Z L$

Z๐Z'8Zレ

SOS' $9 \varepsilon \downarrow-$

66.'9EL
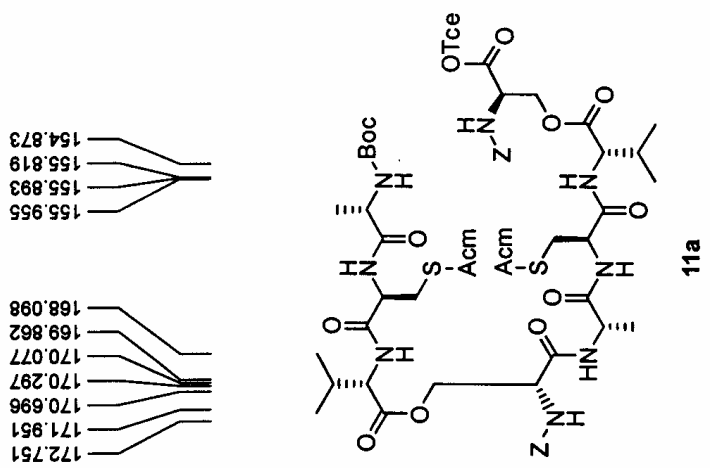


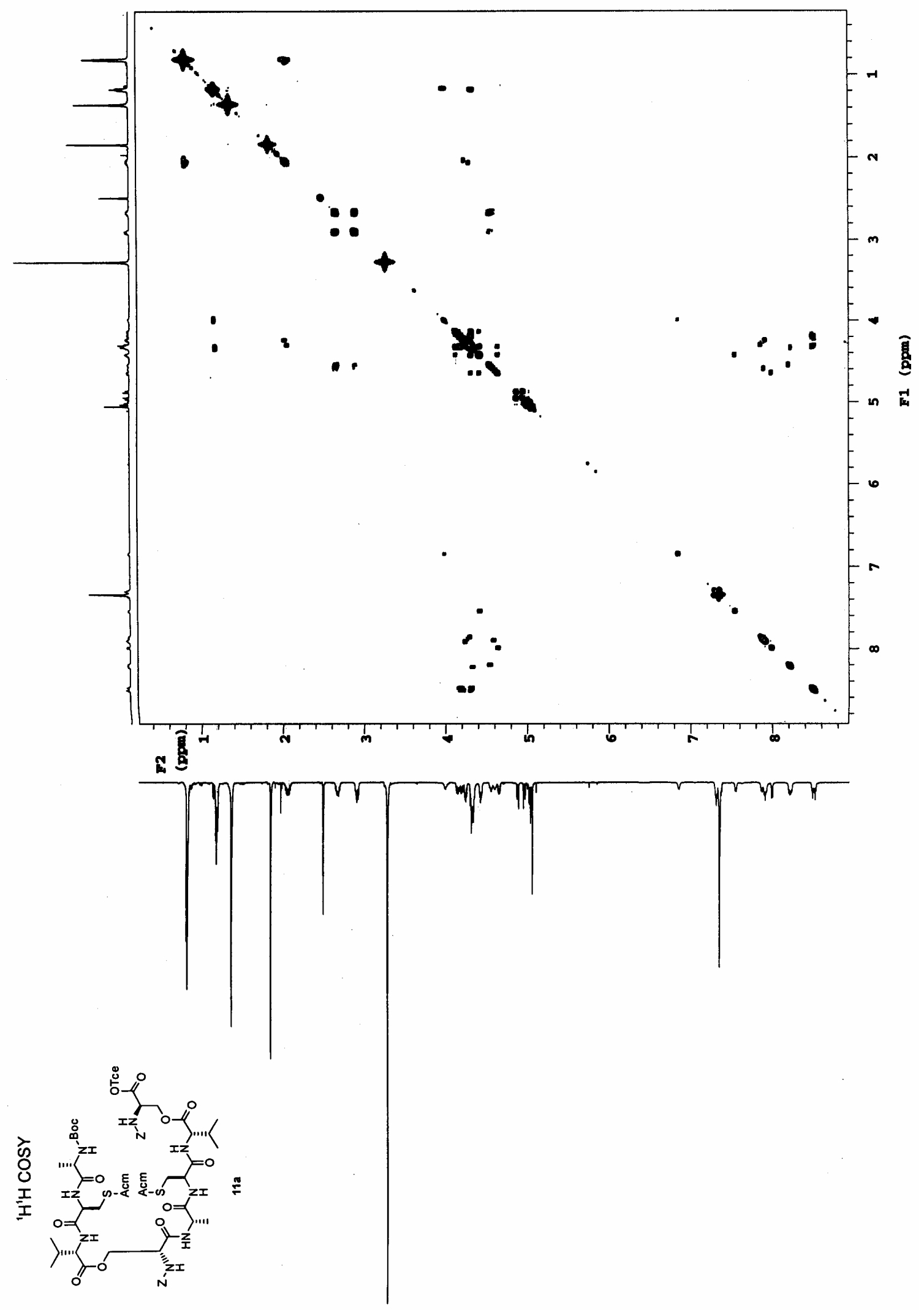




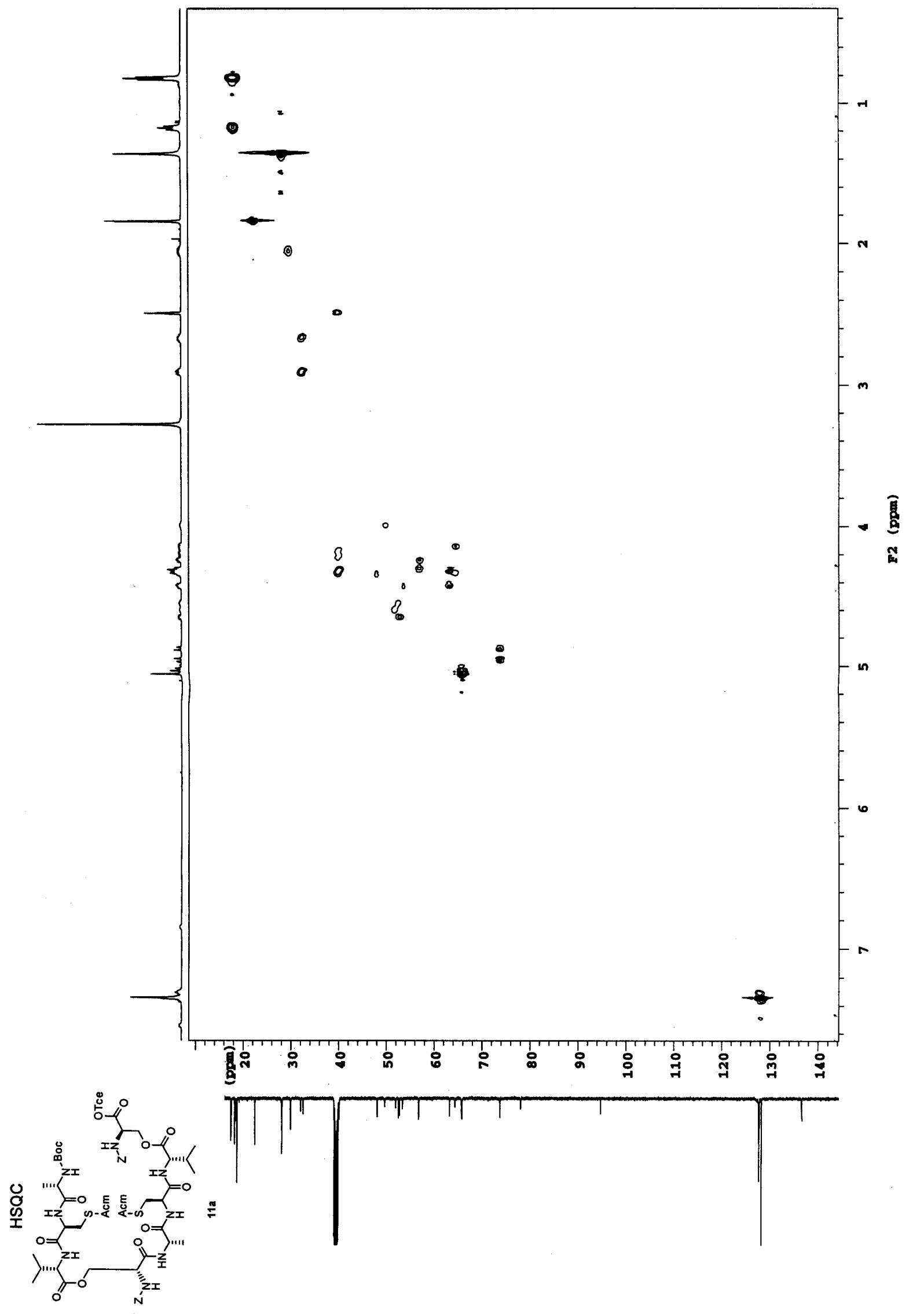




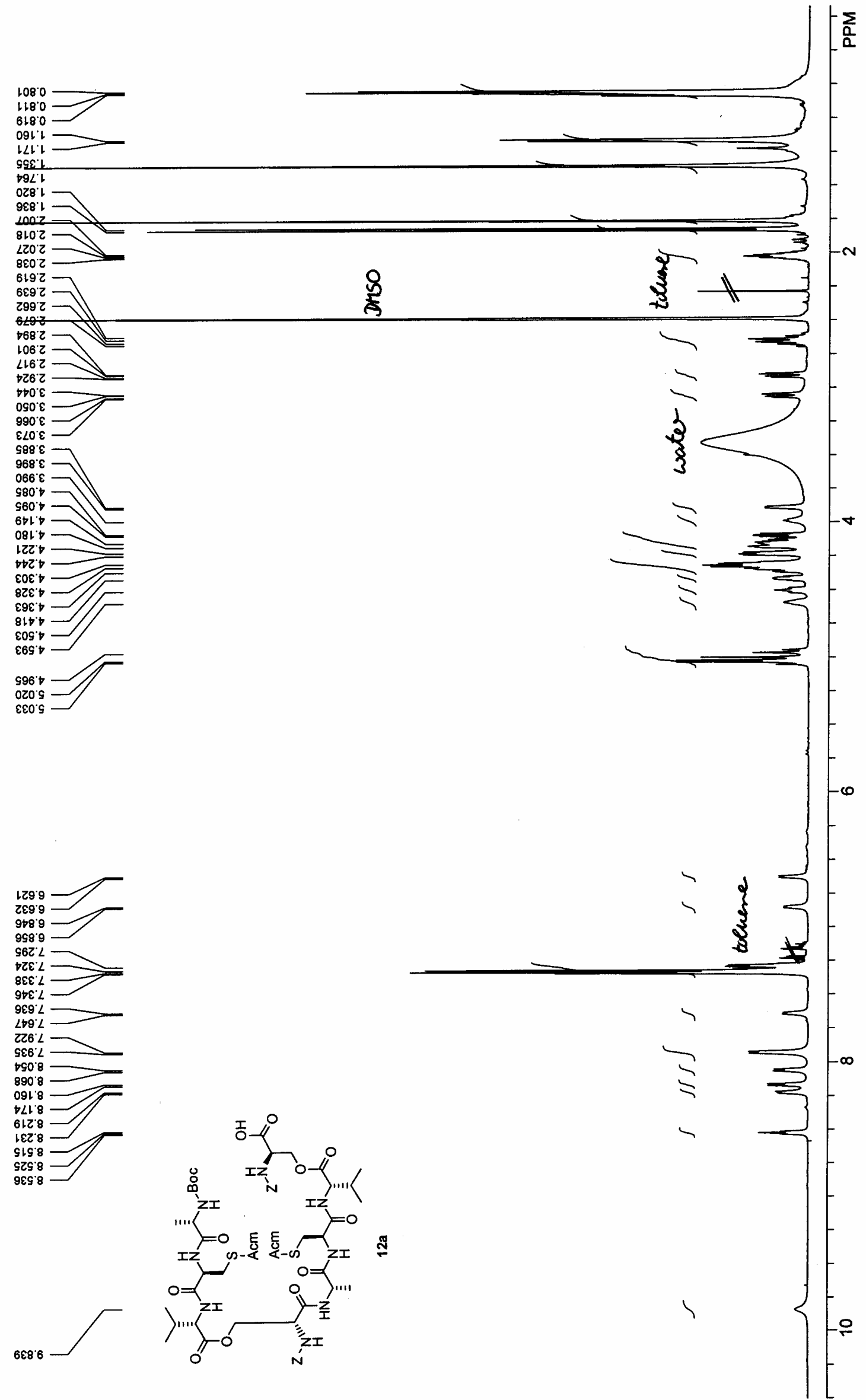



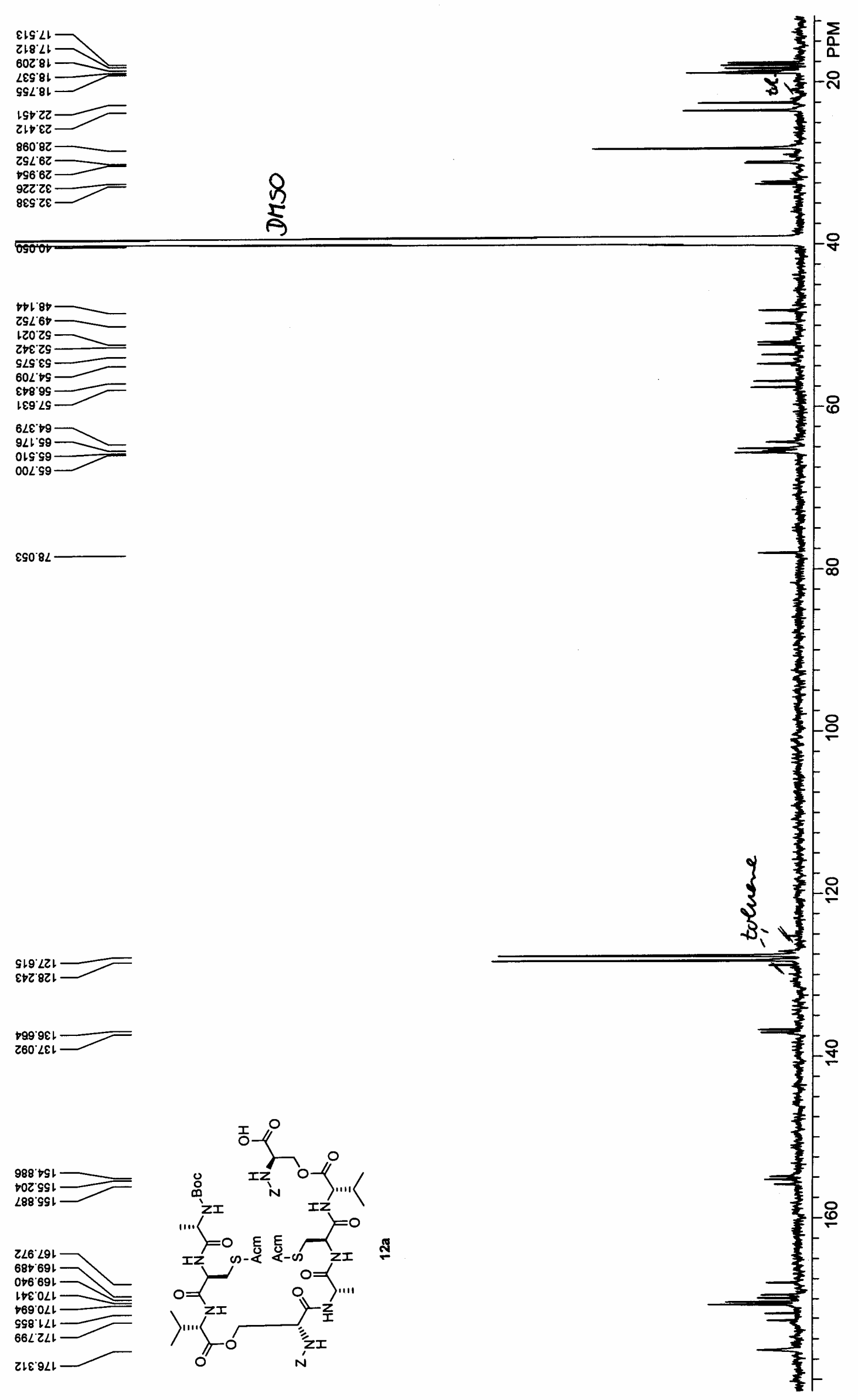

ع90 8L - 


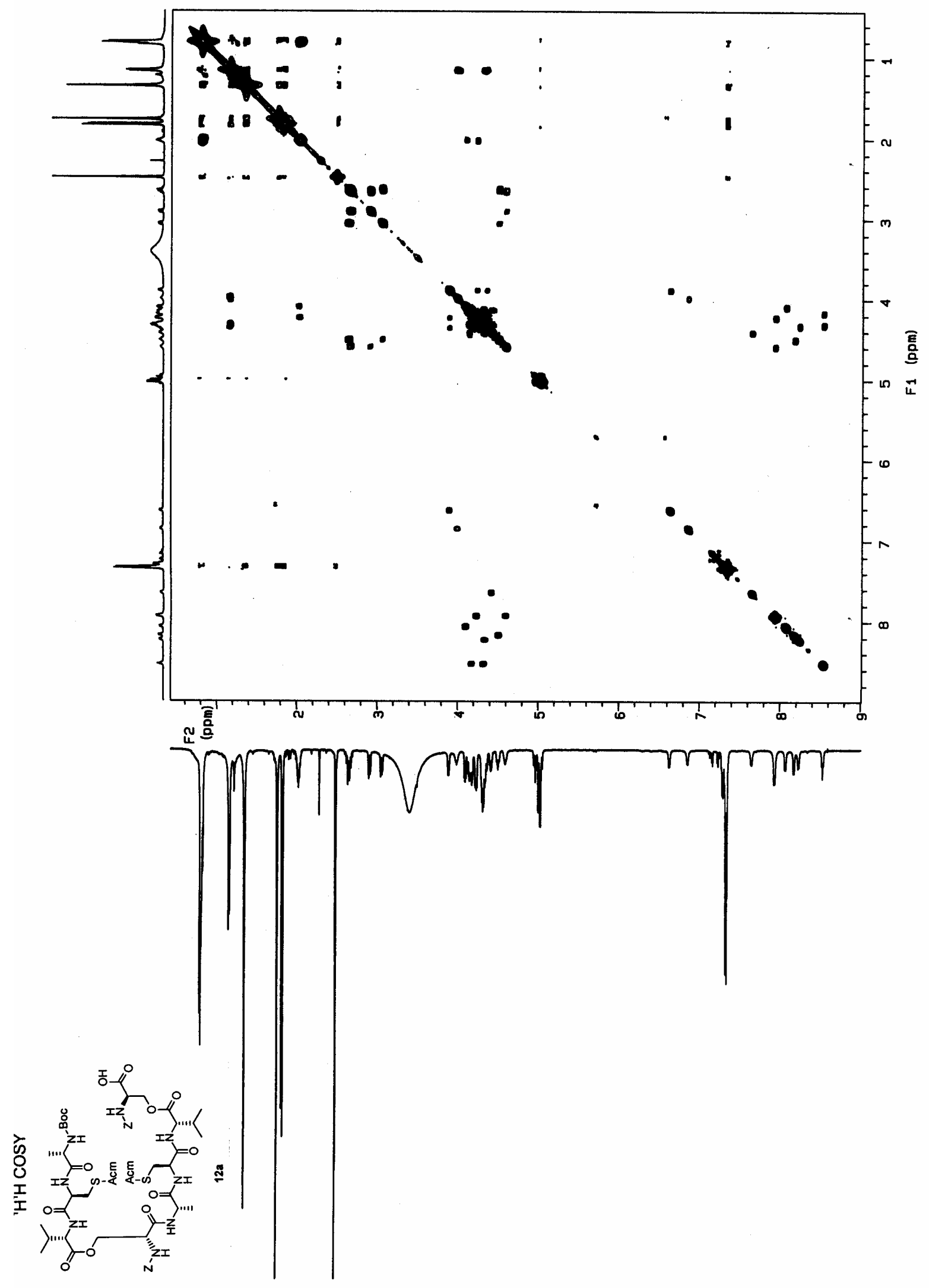




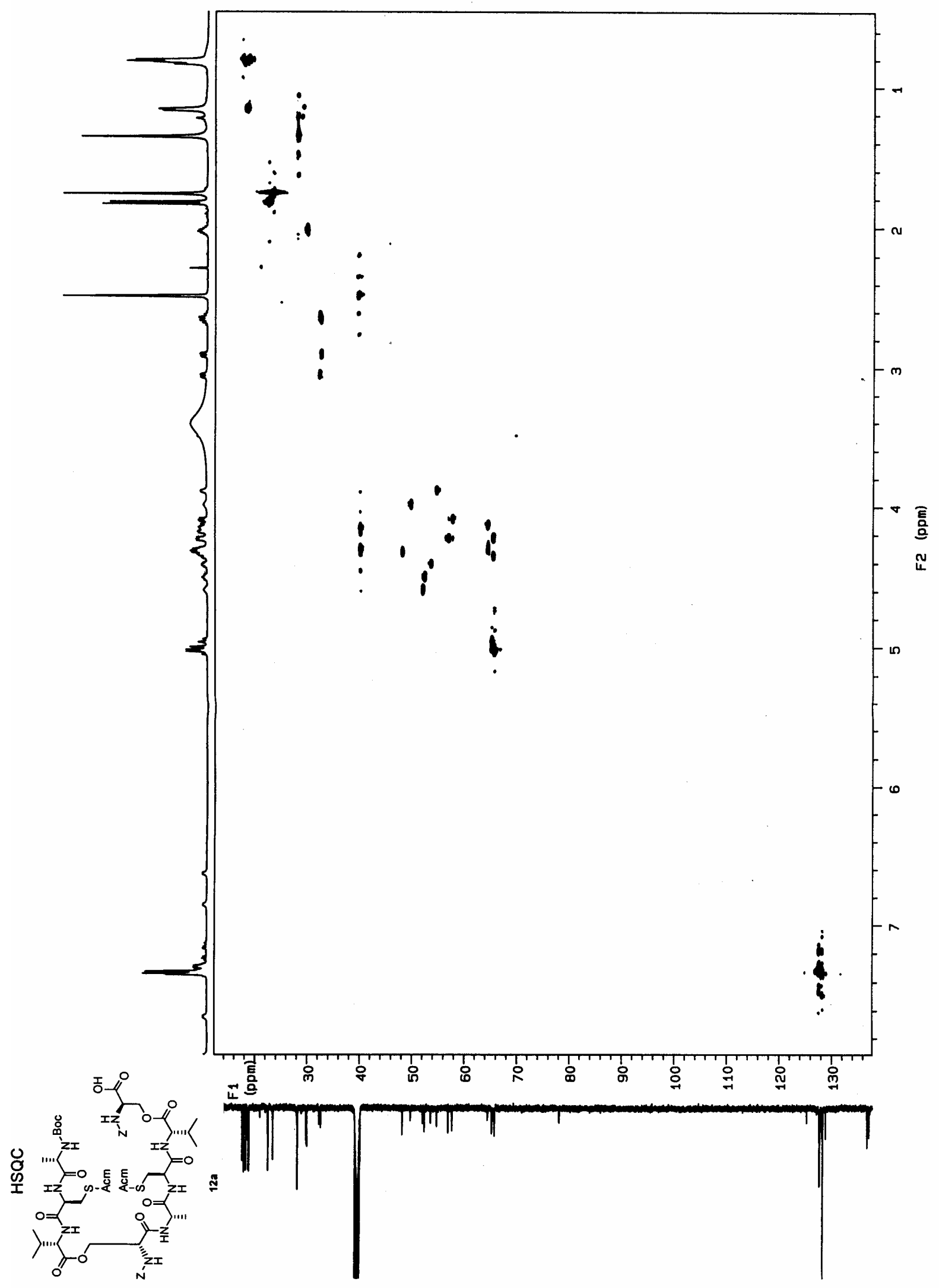


$8 \varepsilon 8^{\circ} 0$

$\varepsilon \rightarrow 8^{\circ} 0$

$6 \$ 8.0$

$\downarrow 98^{\circ} 0$

$\rightarrow 81 \%$

S6L.

$6 \oplus Z \cdot 1$

L9Z' ᄂ

टSE' เ

$1 \rightarrow 0 \cdot 2$

£SO 2

$290 \mathrm{Z}$

$\nabla \angle O^{\circ} Z$

$260^{\circ} 2$

EOL' 2

$0 \angle 8 ' \mathrm{Z}$

$688^{\circ} \mathrm{Z}$

$\checkmark 68^{\prime} \mathrm{Z}$

1162

$9+6 \cdot 2$

896.2

¿Е० $\varepsilon$ -

Z9० $\varepsilon$ -

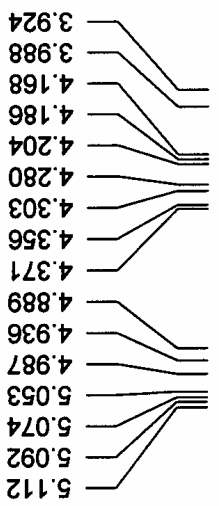

SLS'9

†88. 9

$068^{\circ} 9$

8८ะ $L$

ट $8 \varepsilon^{\circ}$

$669^{\circ} \mathrm{L}$

$\longrightarrow$

ZLLL

$288^{\circ}$

1

$268^{\circ} \mathrm{L}$

2008

510.8

L6L' 8

OLG' 8

SZS 8
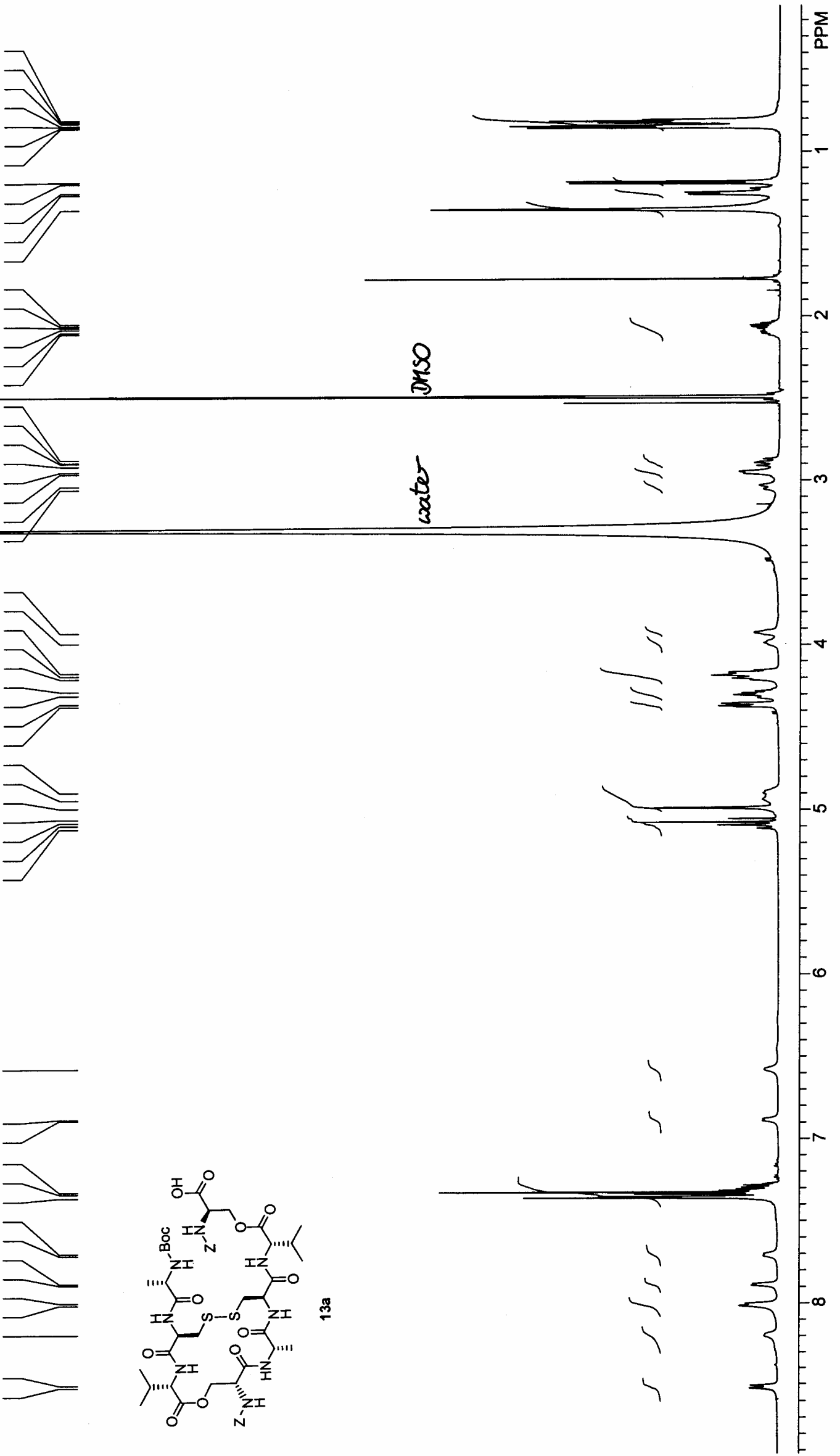

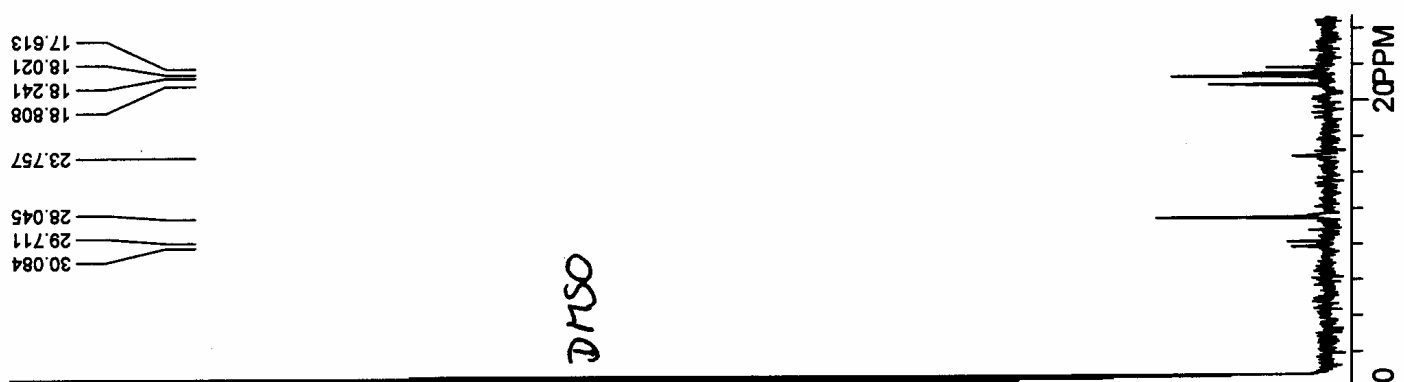

str \&z:

\begin{tabular}{l}
$7968 t-$ \\
921.09 \\
\hline
\end{tabular}

EZLis -

LZZS

$9 \varepsilon 6{ }^{\circ} \& \overline{-} \square$

$Z \downarrow \varepsilon \cdot \angle G=$
$6 Z 1 \cdot 89$

819.79

$\angle 90.99$

S68. 99

$102 \cdot 8 L$

$\angle Z S^{\prime} \angle Z L$

$\rightarrow Z L \angle Z L$

$908<21$

1.1.8Z1

$0<28 Z 1$

z9s' $9 \varepsilon \mathrm{\longrightarrow}$

$560 . \angle \varepsilon L$
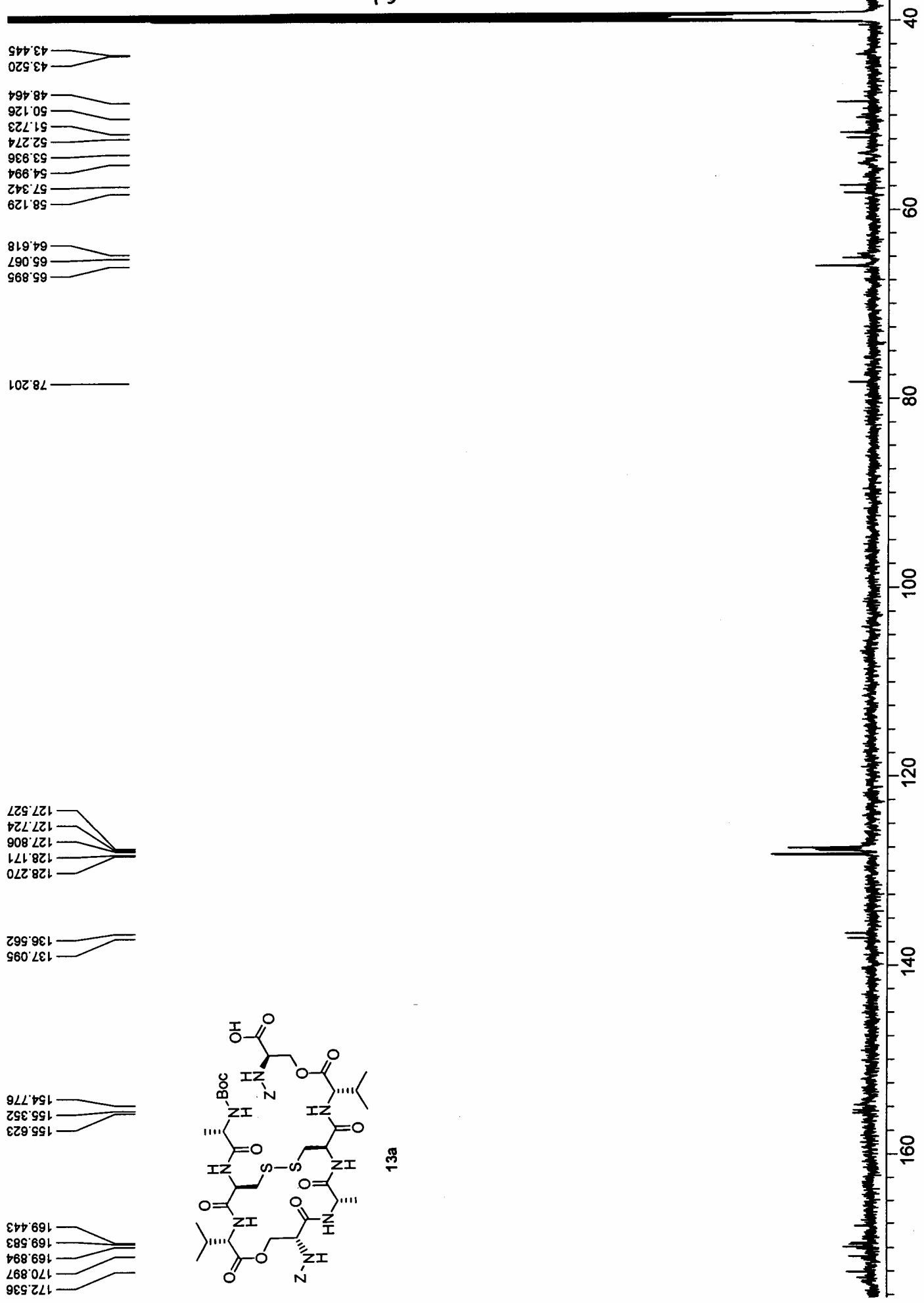


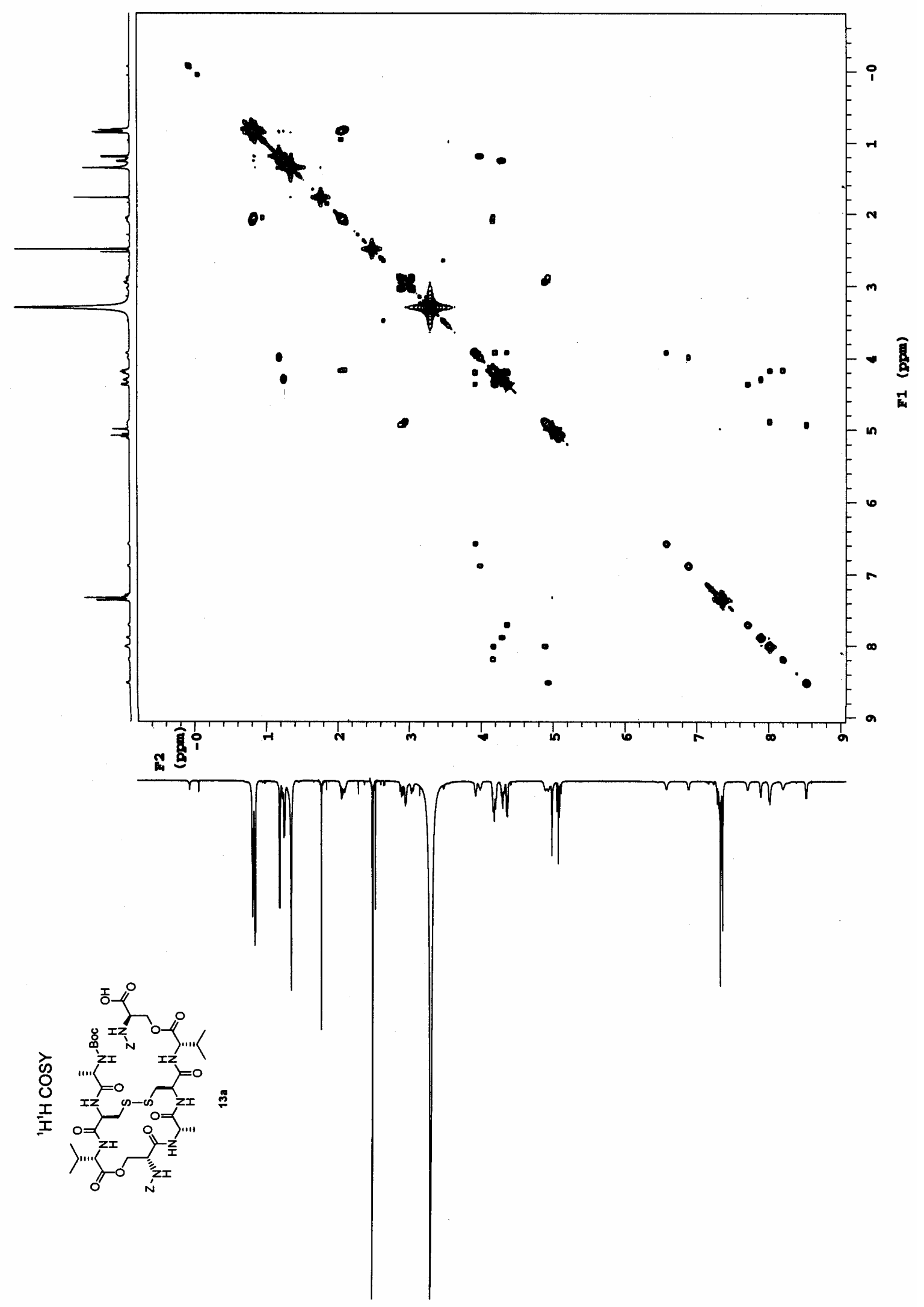




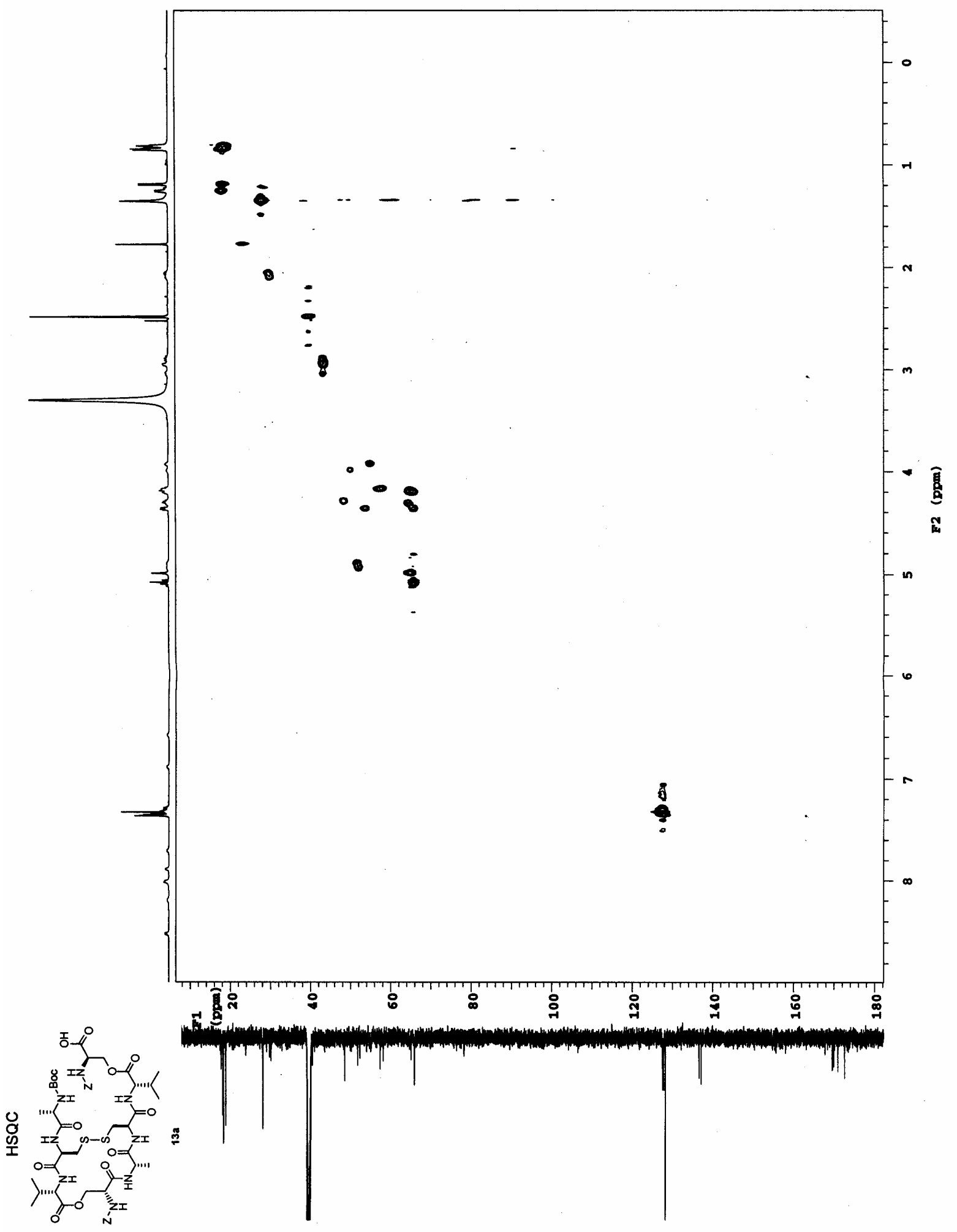



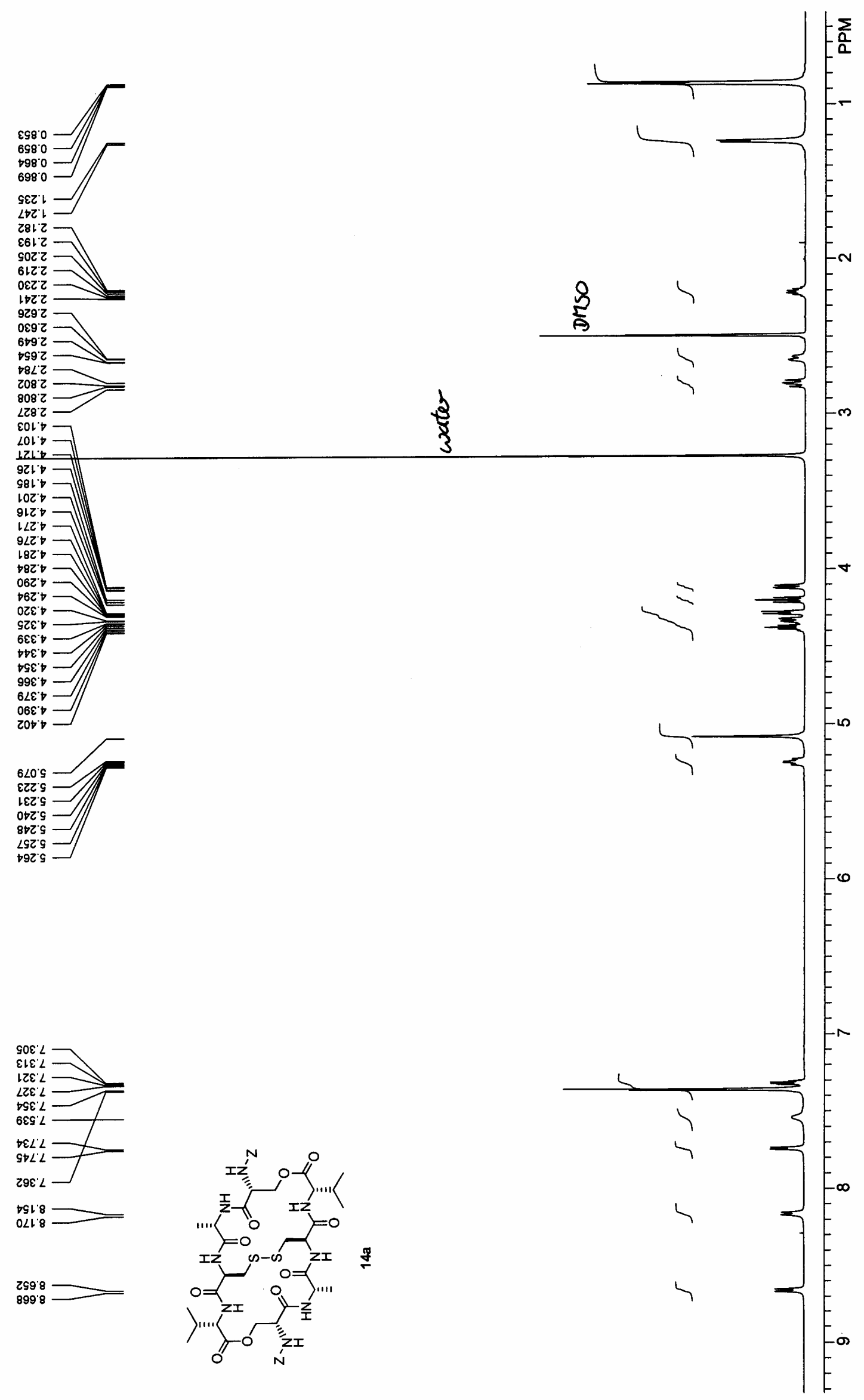
990.81

$\angle L Z{ }^{\circ} 81$

$\angle E 6^{\circ} 8$

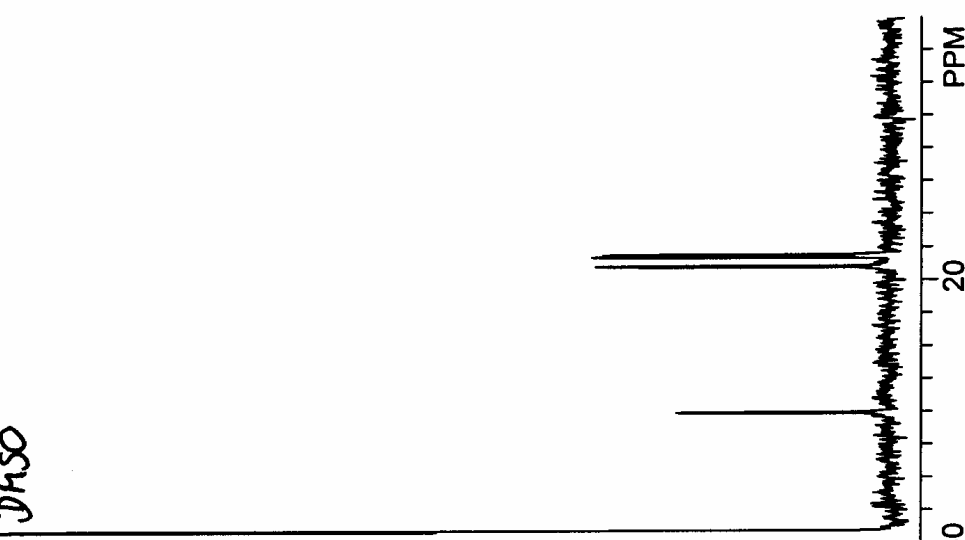

$990^{\circ} 0 \varepsilon$

$\frac{8}{2}$
$\frac{2}{9}$

S66 $L$

ع69'Zs

เE9' $\downarrow 9$

$\downarrow \bullet \varepsilon .89$

$\forall \succ \varepsilon 99$

$8 \angle 8.99$

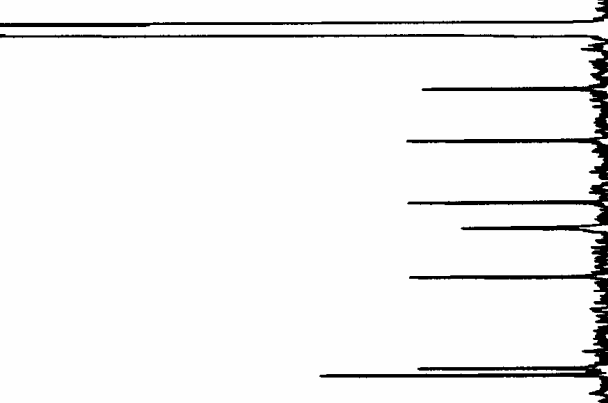

$10 L \angle Z$

$\nabla \angle L \angle Z L$

6978

$0<\mathcal{S}^{\circ} 9 \varepsilon$
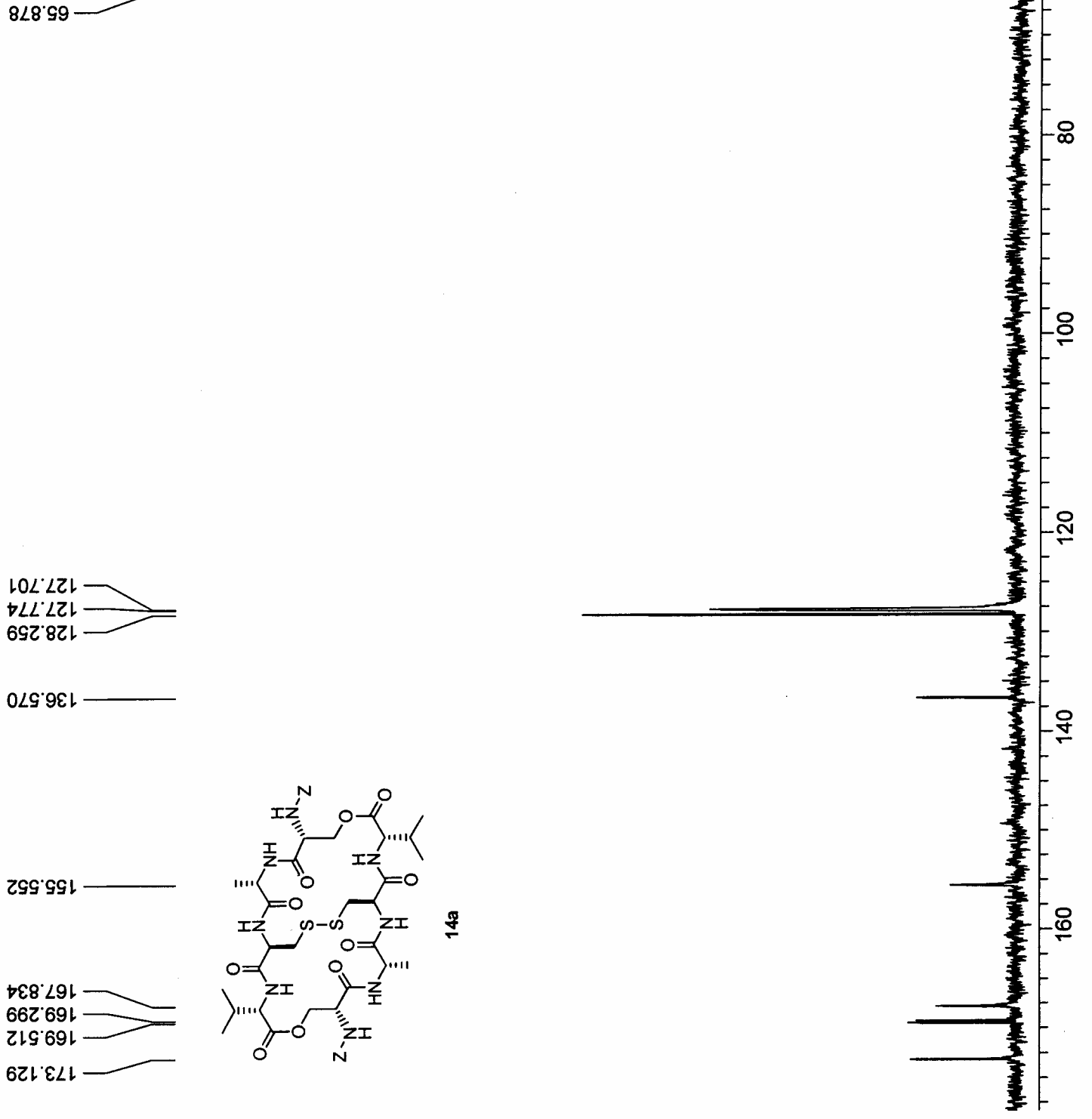


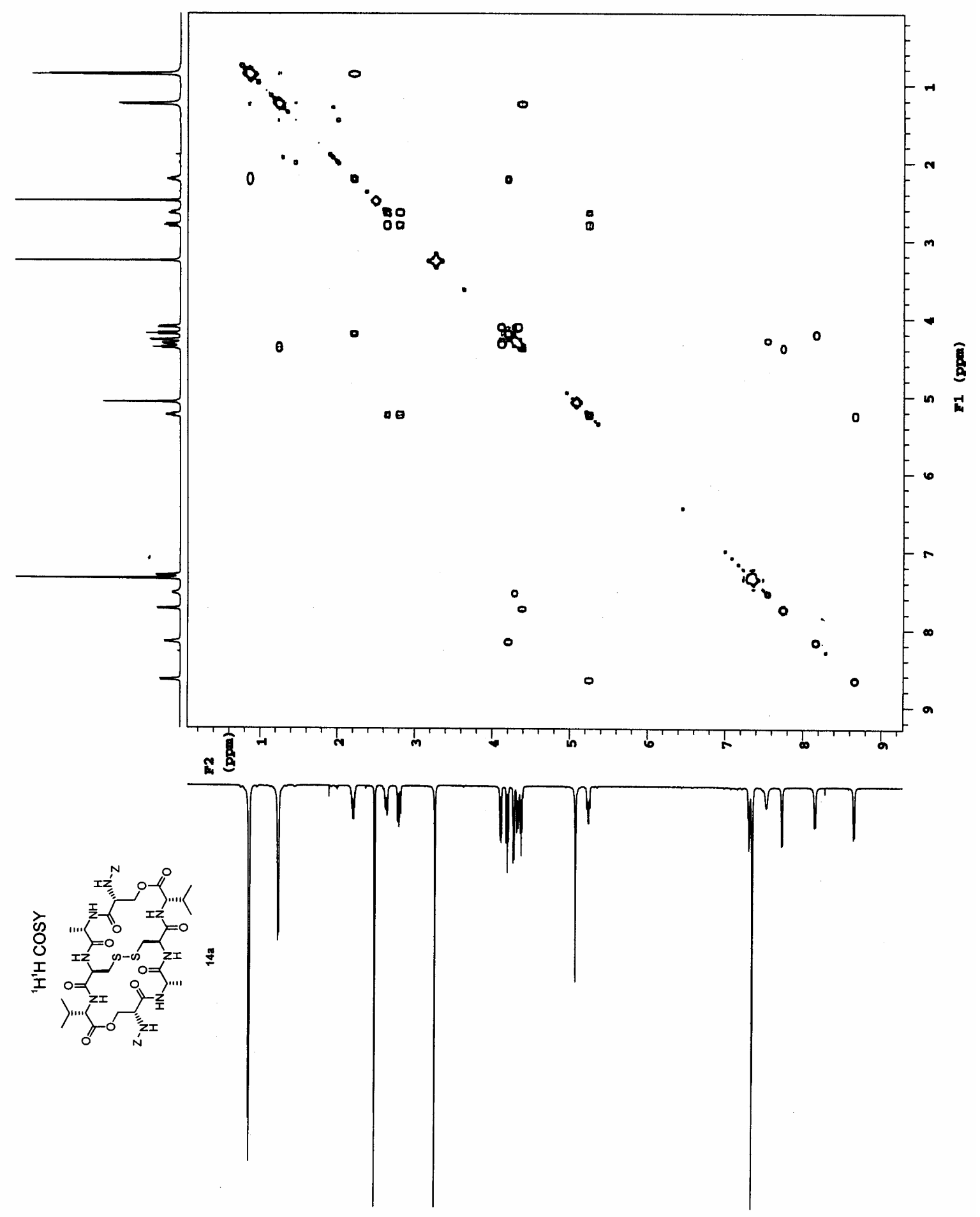




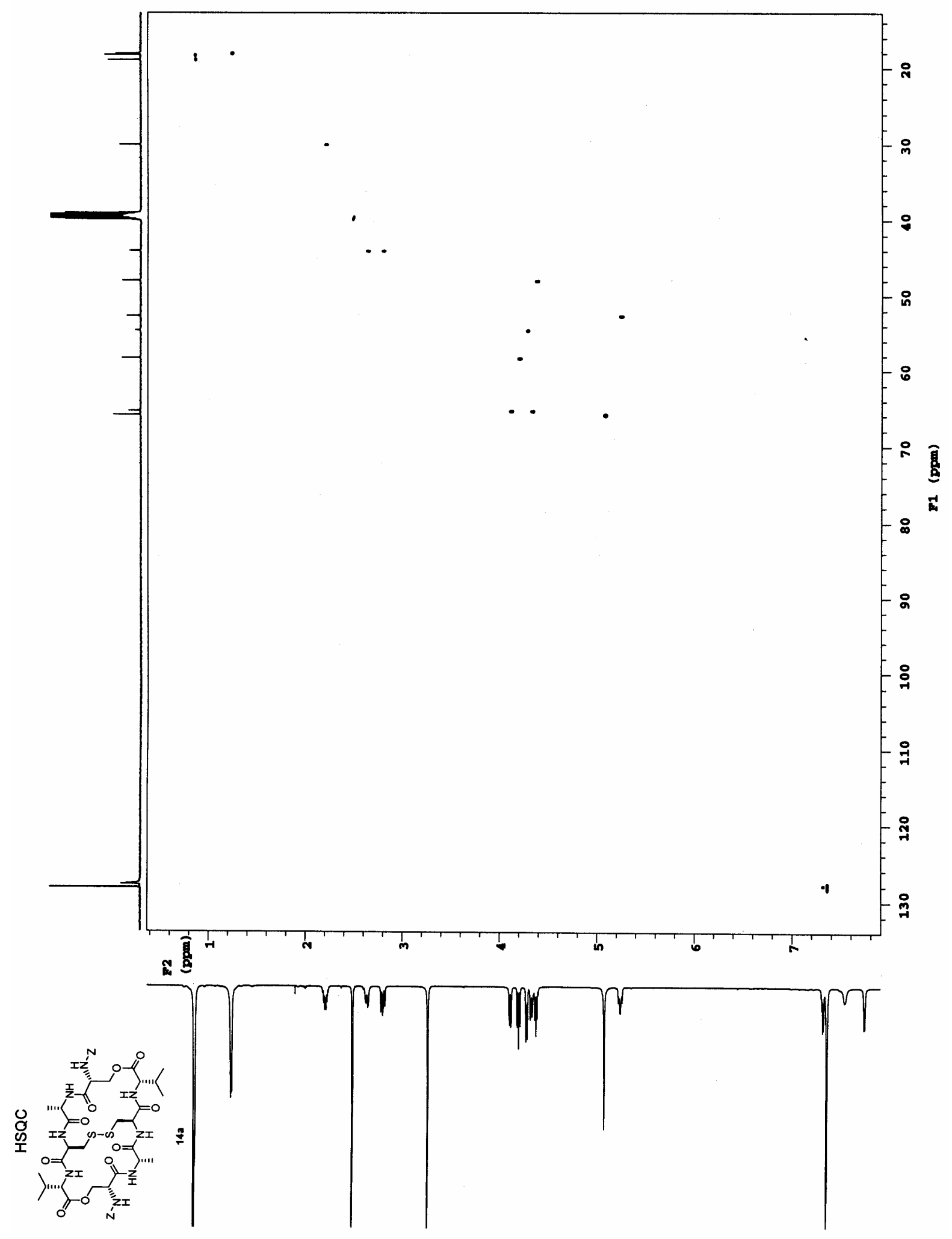




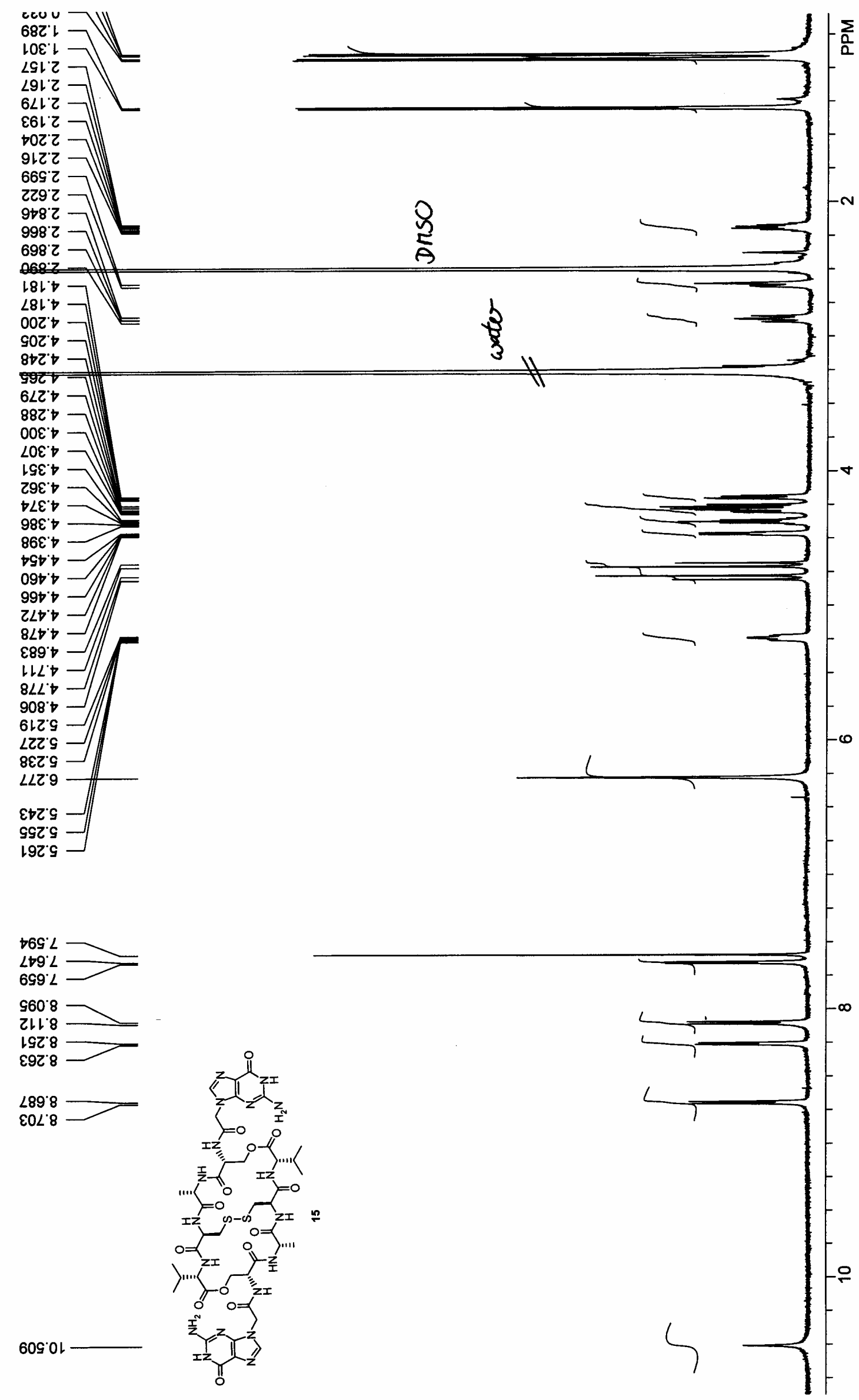


$\varepsilon 98^{\circ} \varepsilon t$

$2 Z 8^{\circ}$

s6z $0 \varepsilon$

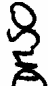

$\downarrow 90^{\circ} 8 \mathrm{t}$

98929

691.89

$+6 L \div 5$

$290.91 /$

$Z \angle 6^{\circ} \angle \varepsilon L$

ISS'Lt

$80 t$ เSL

60tยงเ -

Ett'9sL

$\varepsilon เ 6.99 \vdash$

ع69.69l

$\angle 6 L^{\circ} \varepsilon \angle L$

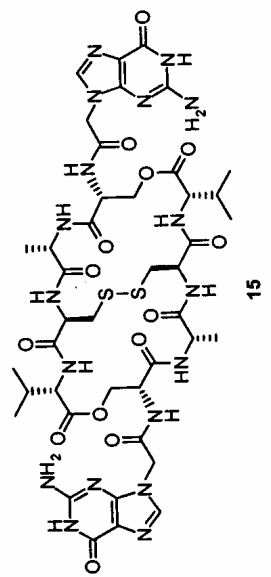




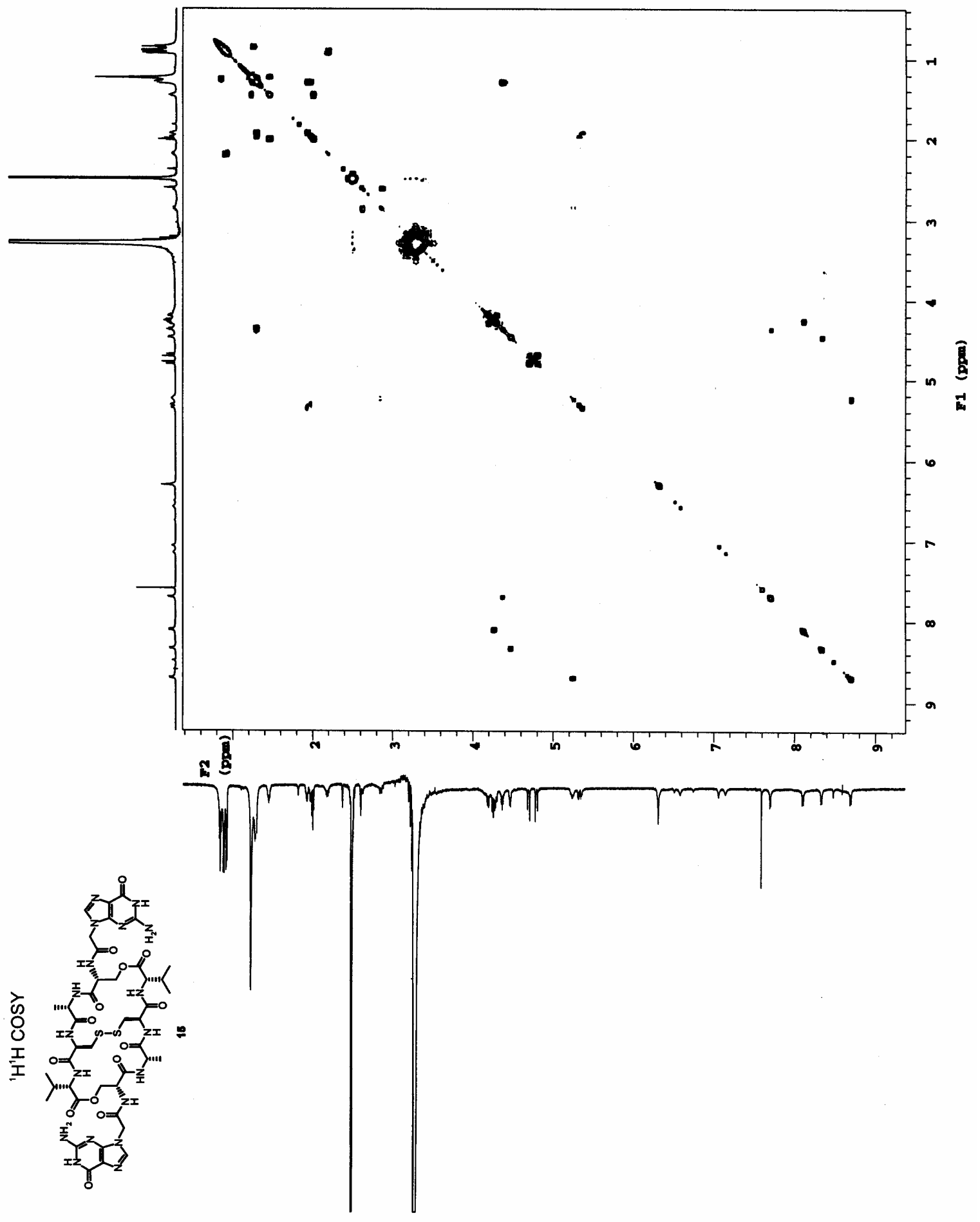



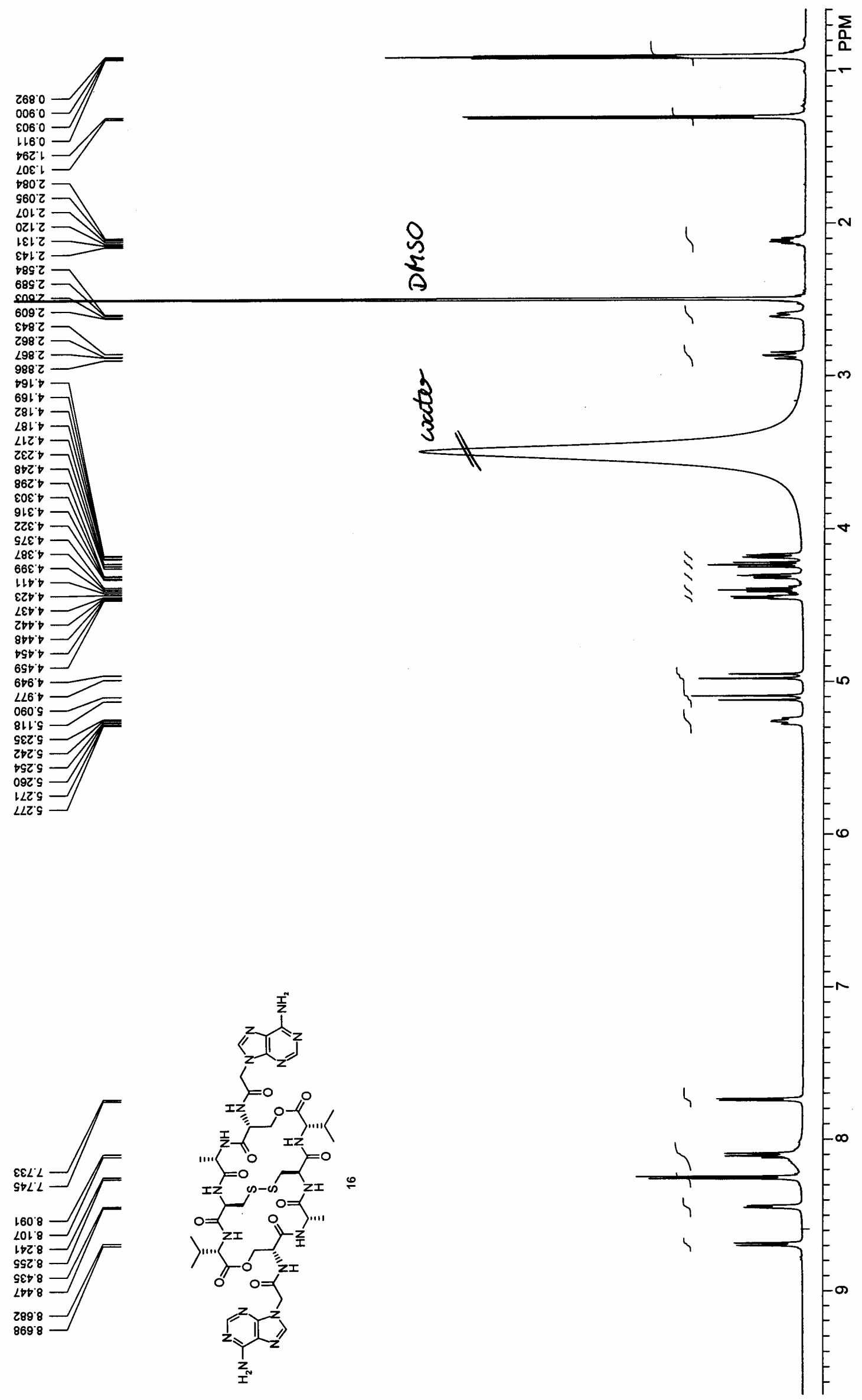
$9 \varepsilon 0.8 \mathrm{~L}$

$69 L^{\circ} 81$

966.8

$\varepsilon 8 \tau^{\circ} 0 \varepsilon$

8

$0166^{\circ} \mathrm{b}$

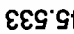

$\varepsilon 66^{\circ} \angle t$

$\angle 89^{\circ} \mathrm{ZS}$

$976^{\circ} \mathrm{ZS}$

เ৮E'89

sZL' $\triangleright 9$

91081
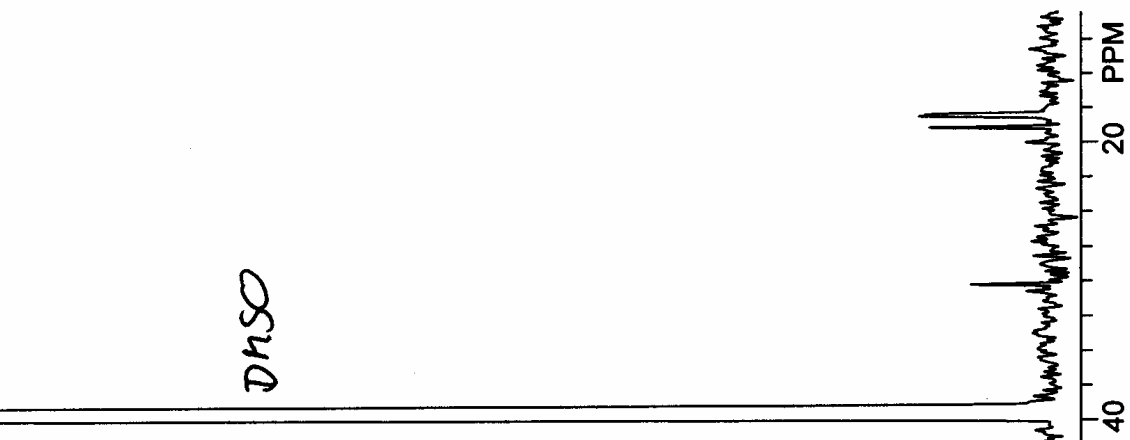

요
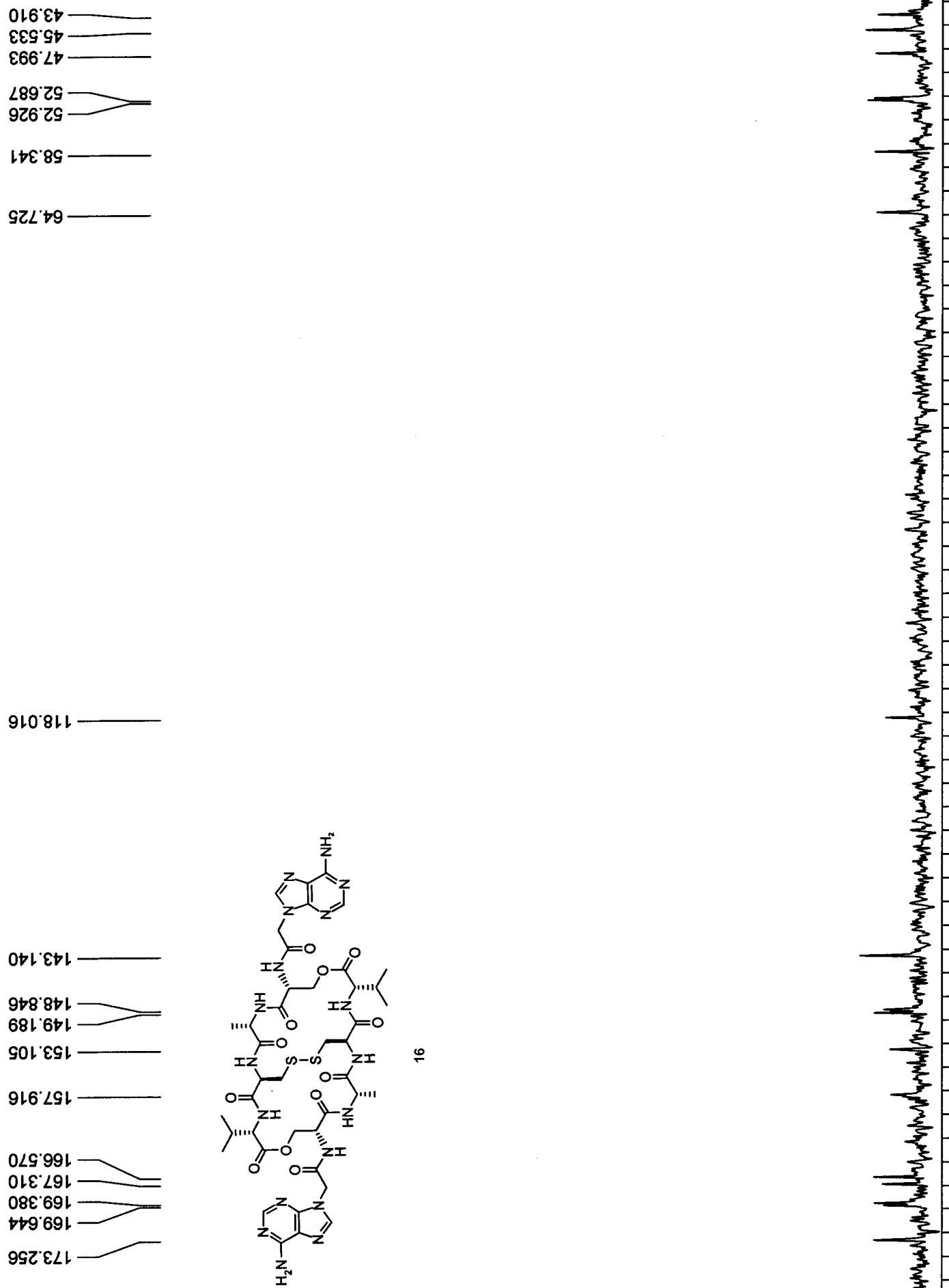


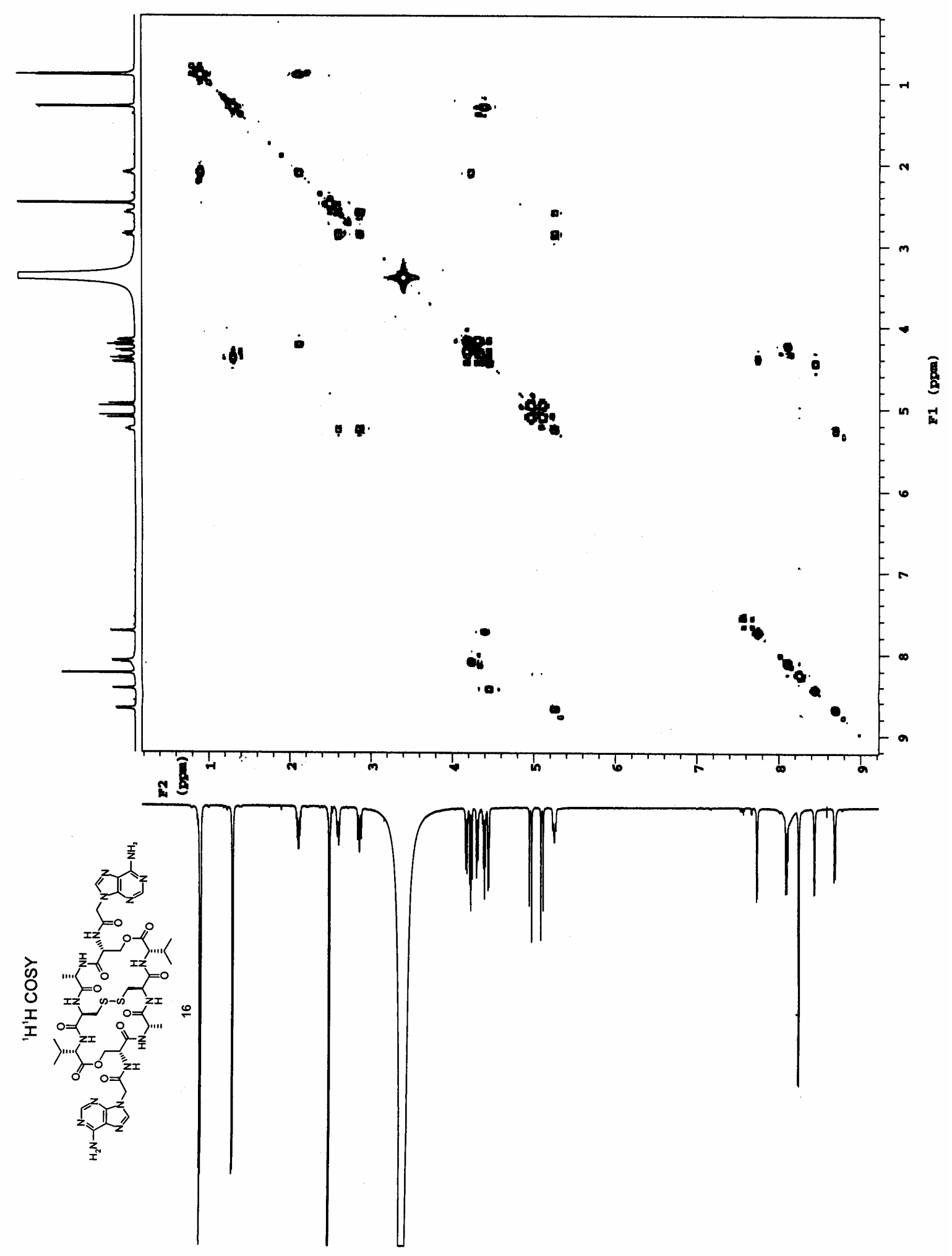




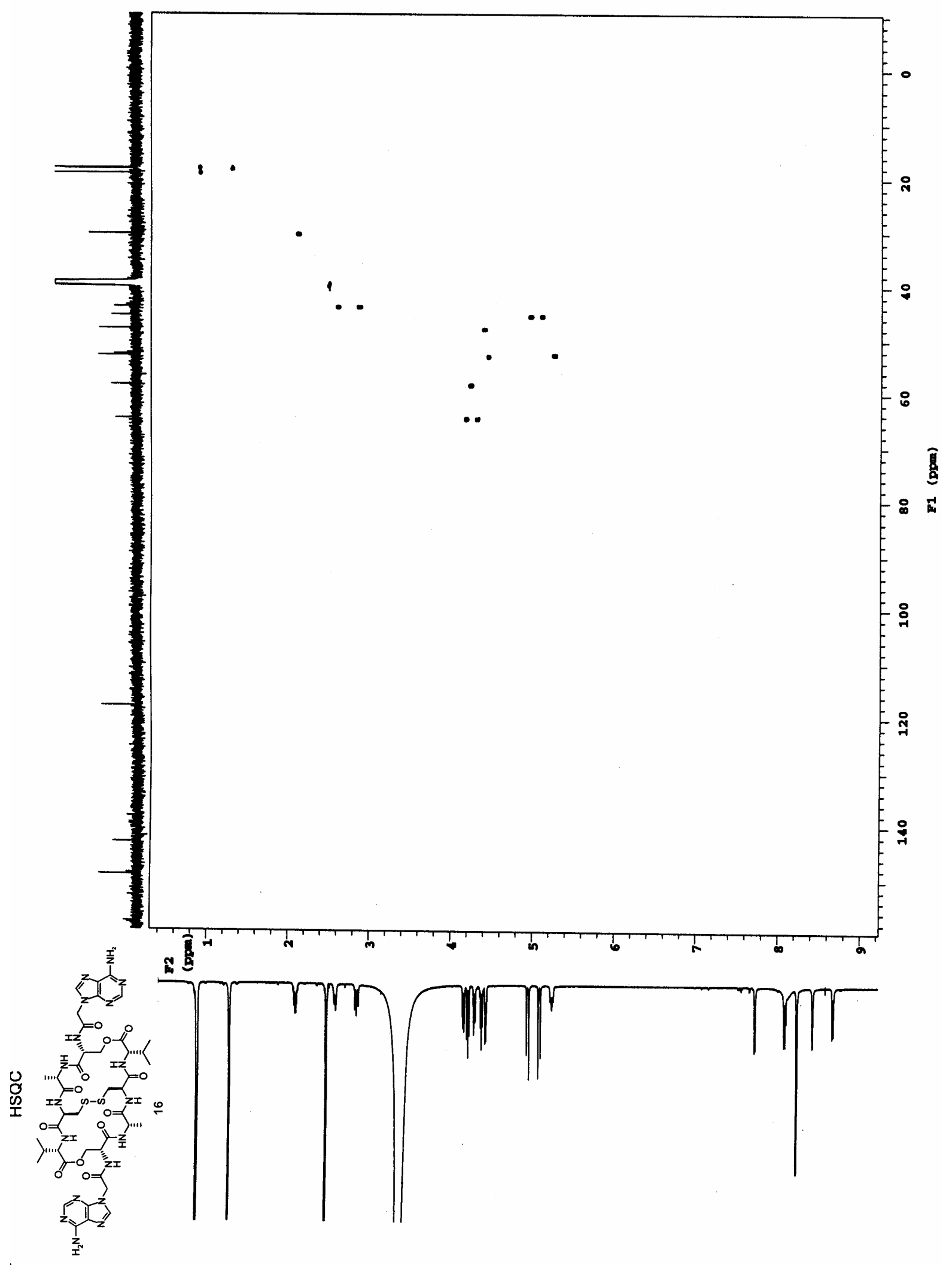




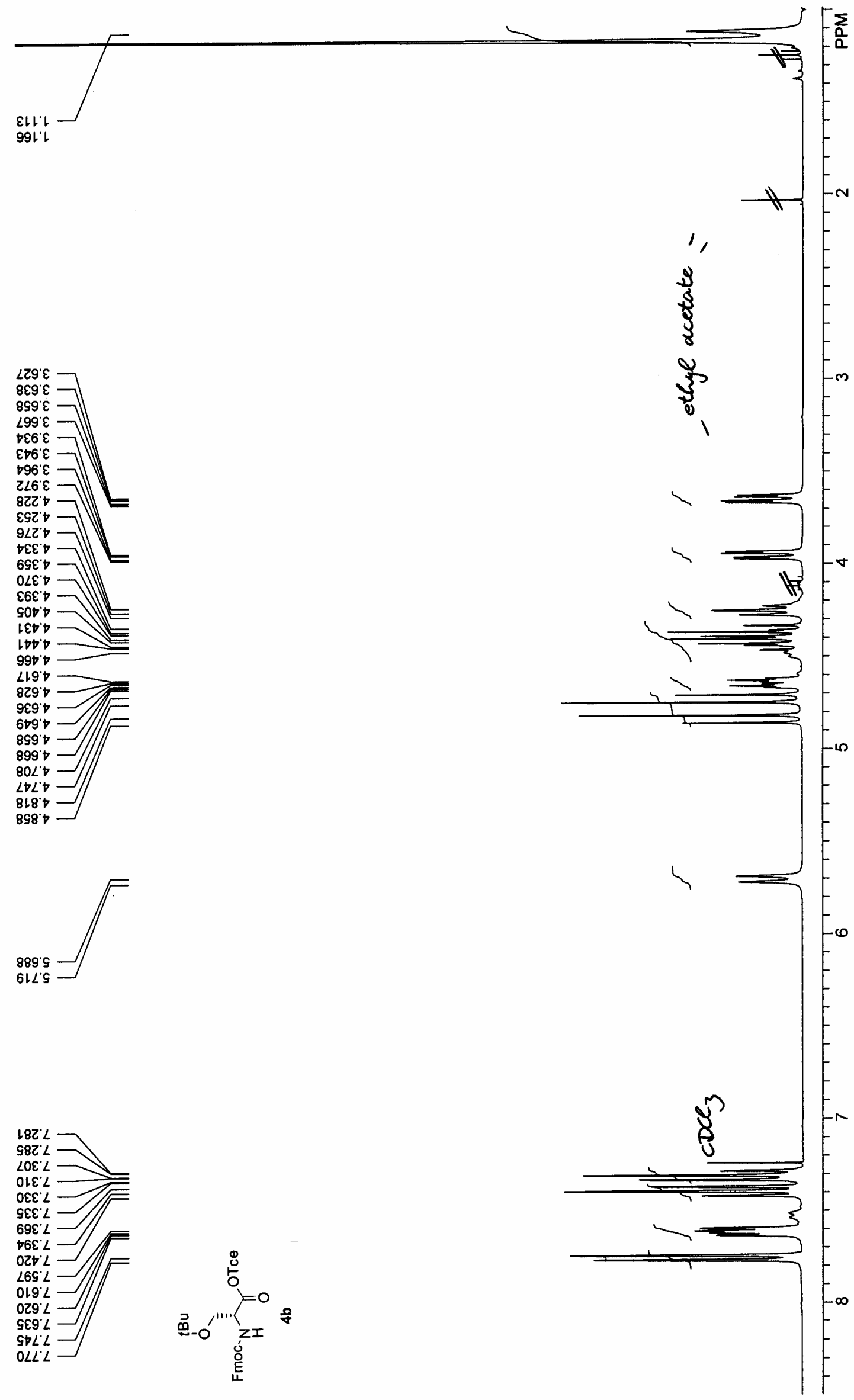


$\downarrow เ \varepsilon<2$

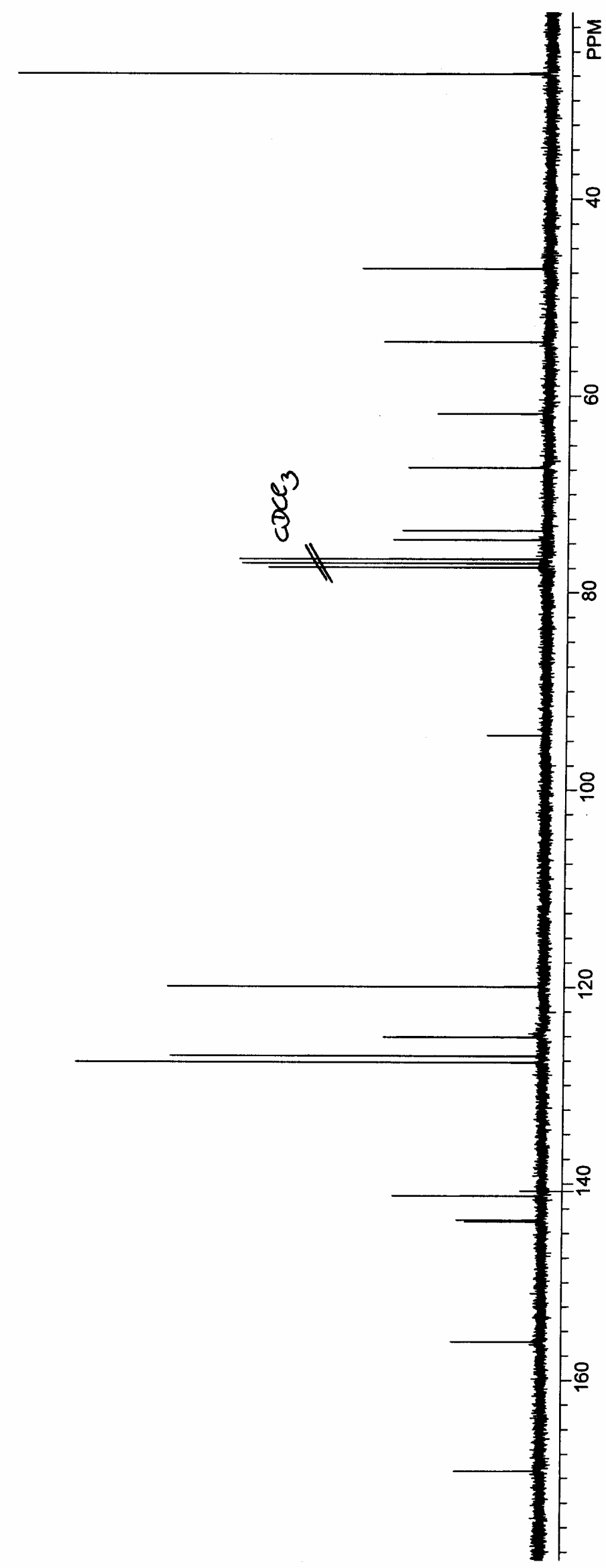

$001^{\circ} 9 \mathrm{SL}$

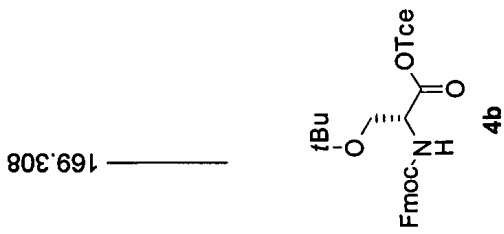

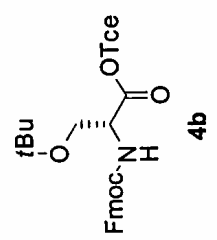

601.921

$610.2 Z 1$

$\varepsilon+02 Z 1$

$\nabla Z Z \cdot t+$

699' $\bullet \triangleright 1$

$\varepsilon \varepsilon 8$ ¿ヤレ - 
976

$6 \multimap 6$.

เ96' $\varepsilon$

$\angle 96^{\circ} \varepsilon$

$660^{\circ} \mathrm{t}$

LIL'

861.5

602 '

$12 Z+$

$\neg 8 \varepsilon \triangleright$

Lot't

Eเt

9Zt॰

LEt'

sGt't

GLS'D

$289^{\circ} t$

$685^{\circ} t$

$\varepsilon 0 L^{\prime \prime} \downarrow$

EZL'

$988^{\circ} \downarrow$

906 '

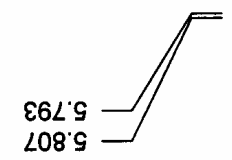

$\succ 82$

$96 Z$ L

$60 \varepsilon$ ' 2

$\varepsilon L \varepsilon^{\prime} L$

GLE'L

$98 \varepsilon^{\prime} L$

$\angle 8 \varepsilon^{\prime} \angle$

$86 \varepsilon^{\prime} L$

OOt'L

9LG' $\angle$

S69' $L$

$\mathrm{Z} L L$

$\forall S L L$

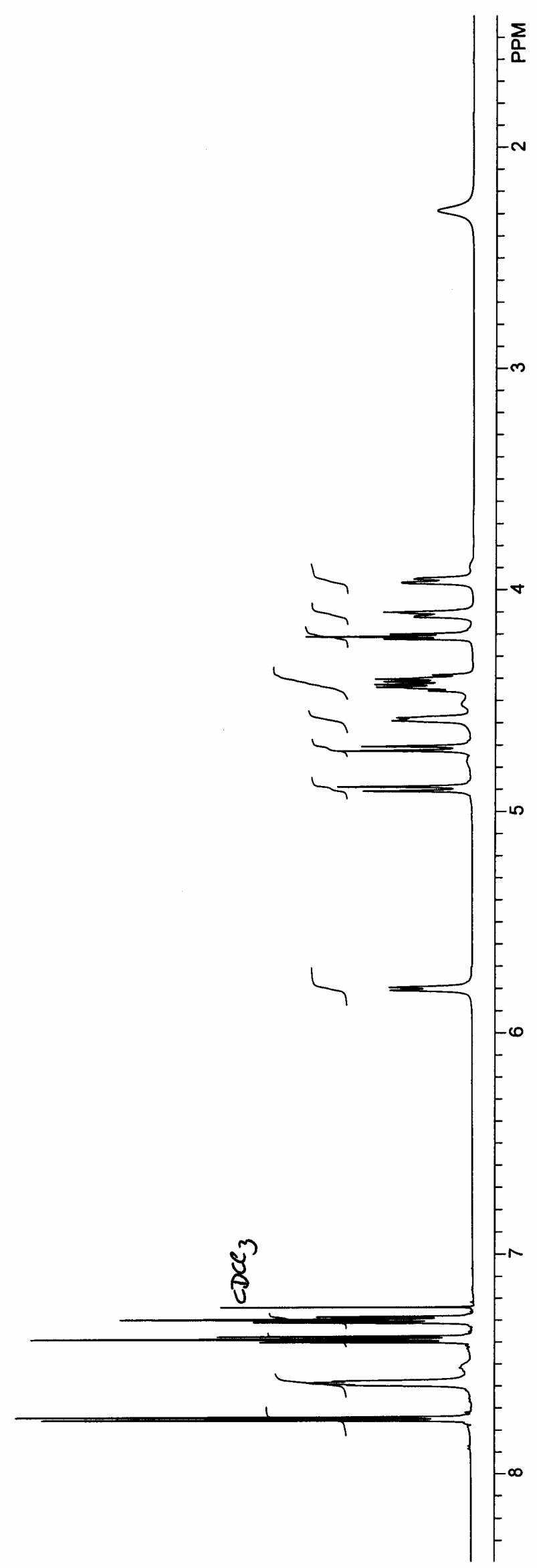


$1 L O \angle t$

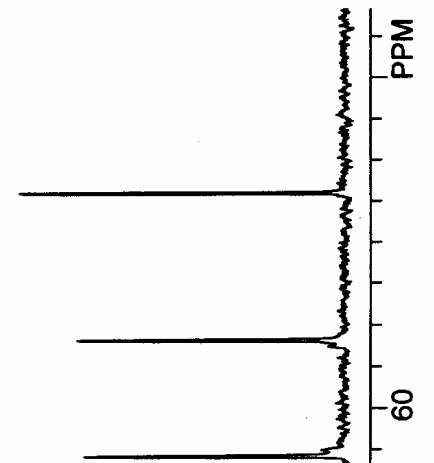

$868 \cdot 29$

$897<9$

$s 8 b^{\circ}+L$

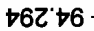

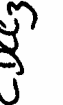

$866^{\circ} 61$

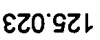

$280^{\circ} \angle Z L$

○

$9 L Z \cdot t \downarrow$

L $\angle$ E Et -

Z69'Et।

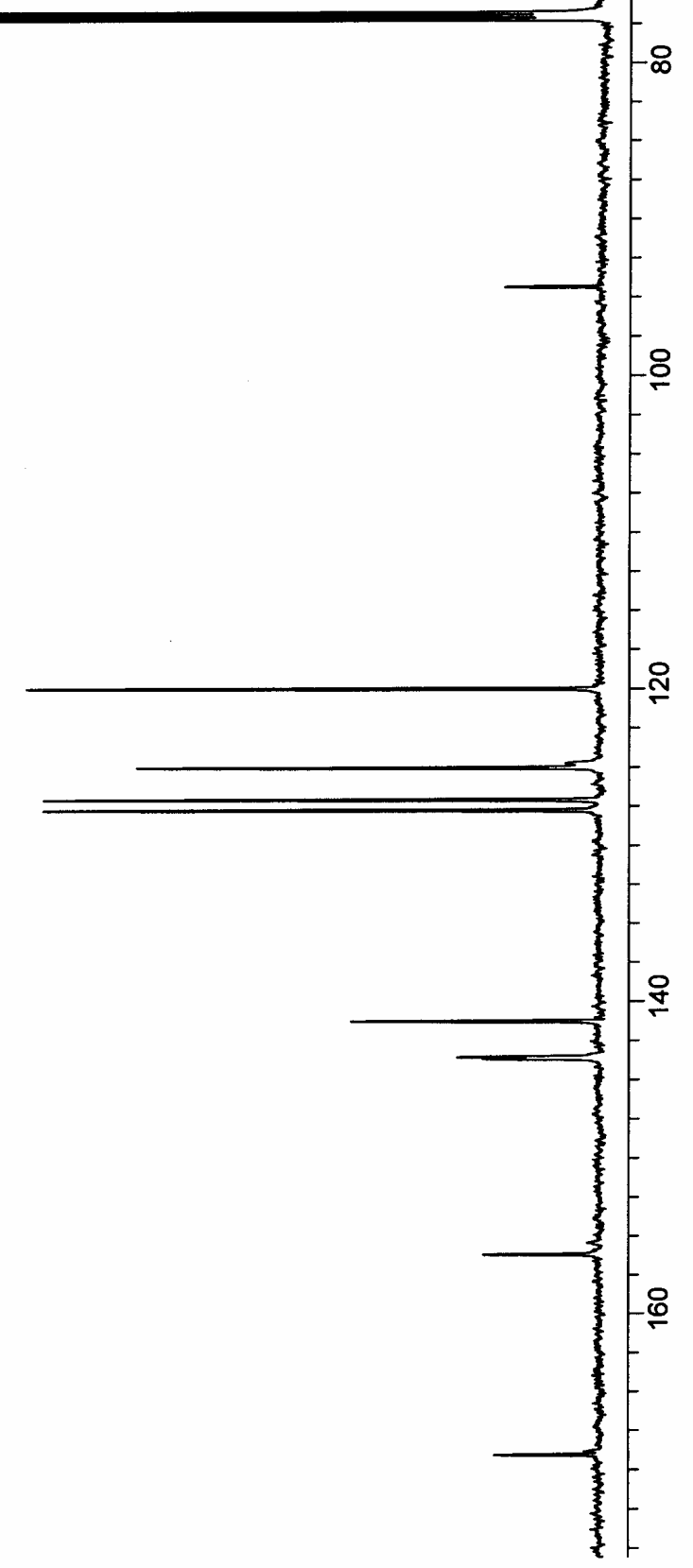



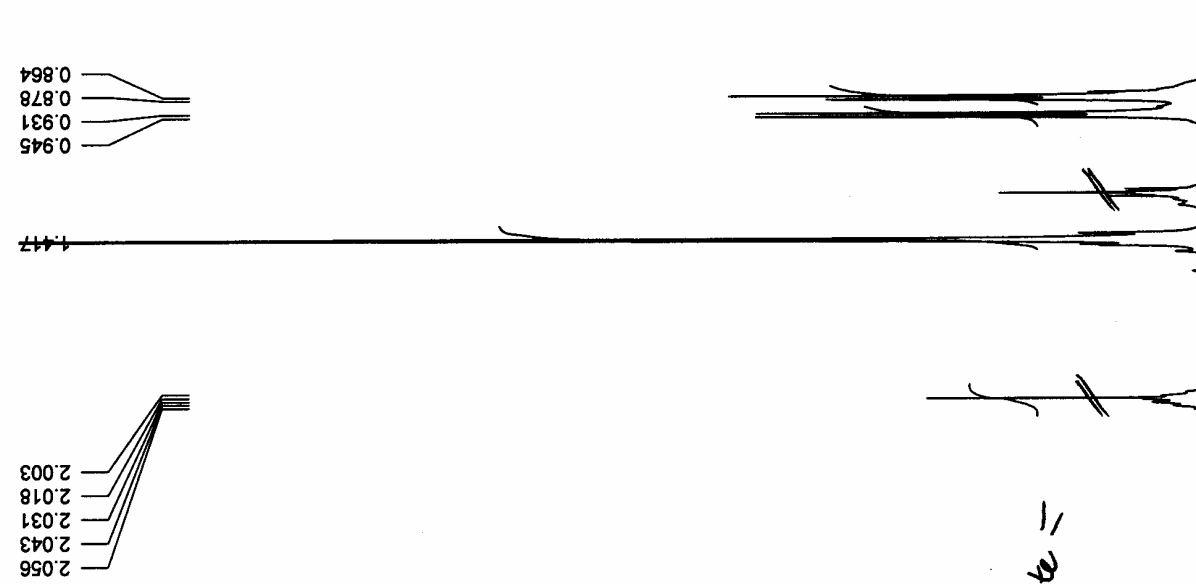

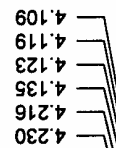

$\forall \forall Z^{\natural}$

เЬE'

Z9E'

$98 \varepsilon^{\circ} \nabla$

ZOt

$\varepsilon z t \circ-1$

0 ' $\Rightarrow$

O8C

(6)

$\varepsilon 69$

Lह9'

5996

$699^{\circ} \mathrm{b}$

$8 E L \circ$

Z9L' $\mathrm{G}$

LE8 $t$

8ह8 $678^{\circ} \circ$

$6 \varepsilon 6$ '

$996 \circ$
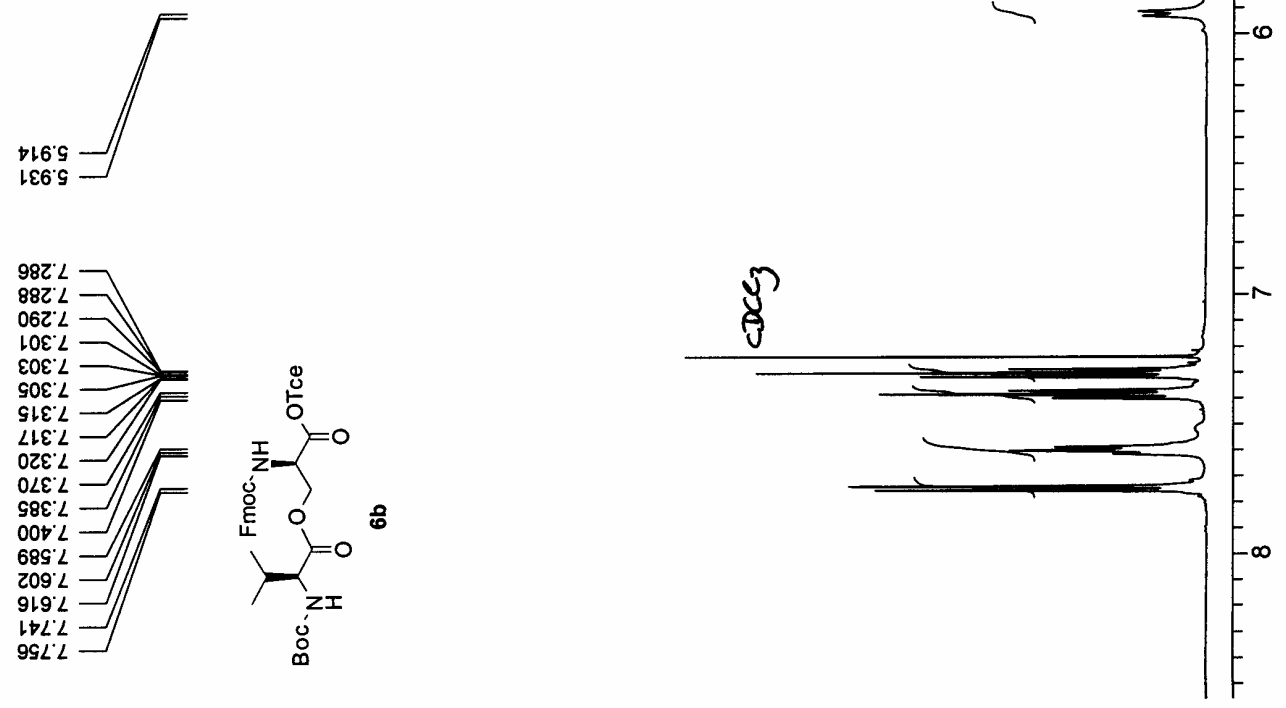
$90 \angle \angle L$

896.81
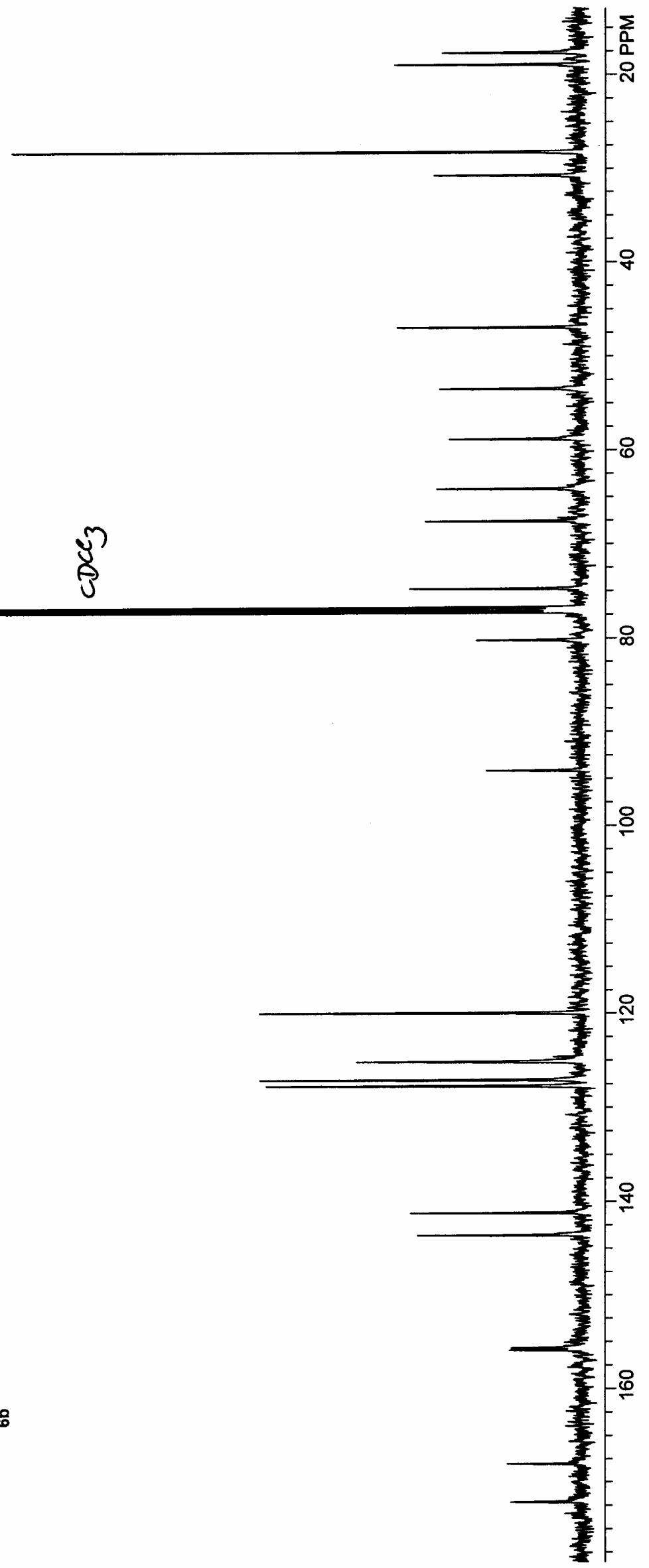

$926.6 \mathrm{~L}$

$\triangle Z I \cdot G Z L$

$990^{\circ} \mathrm{LL}$

$\angle 89^{\circ} \angle Z$

ริ

81:08

ع เ เ6

s9s. 29

$\angle L D L L$

$\varepsilon 0 Z \cdot เ$

†เด है
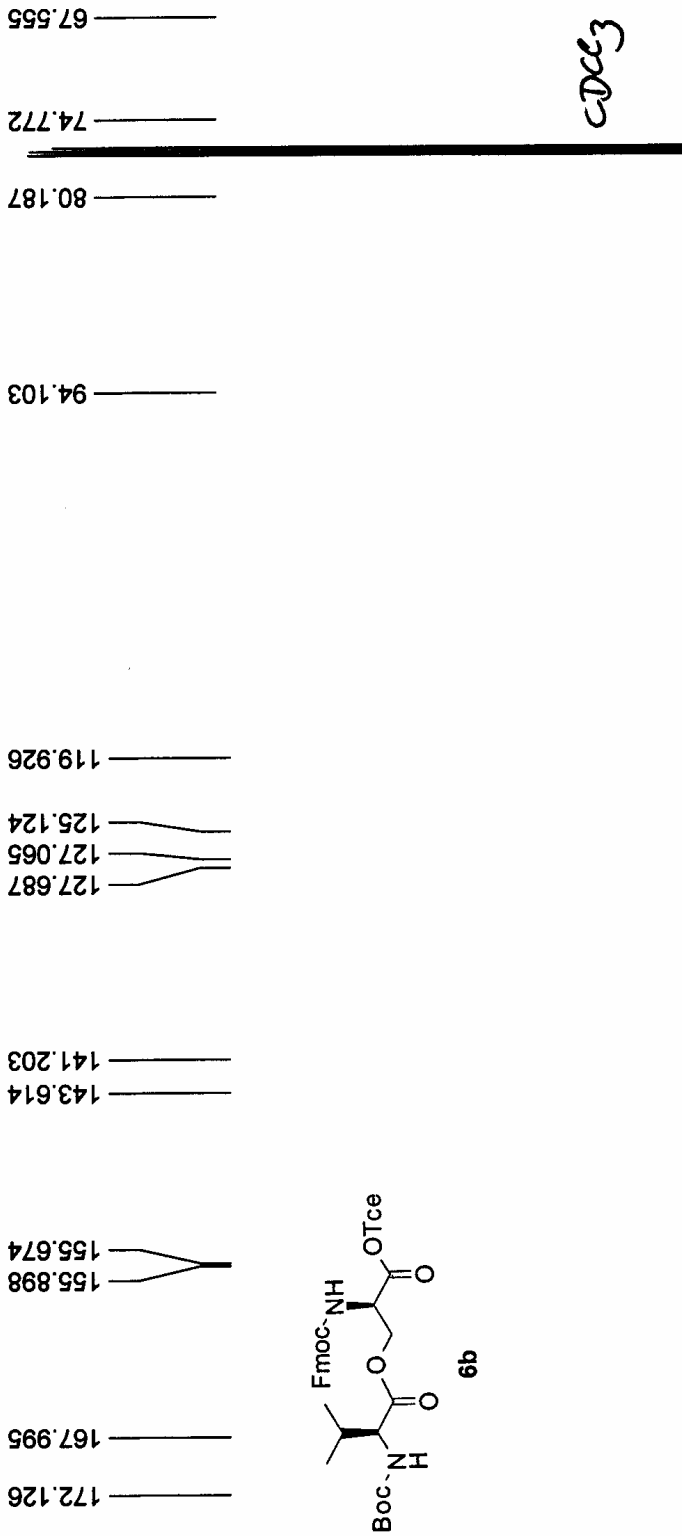

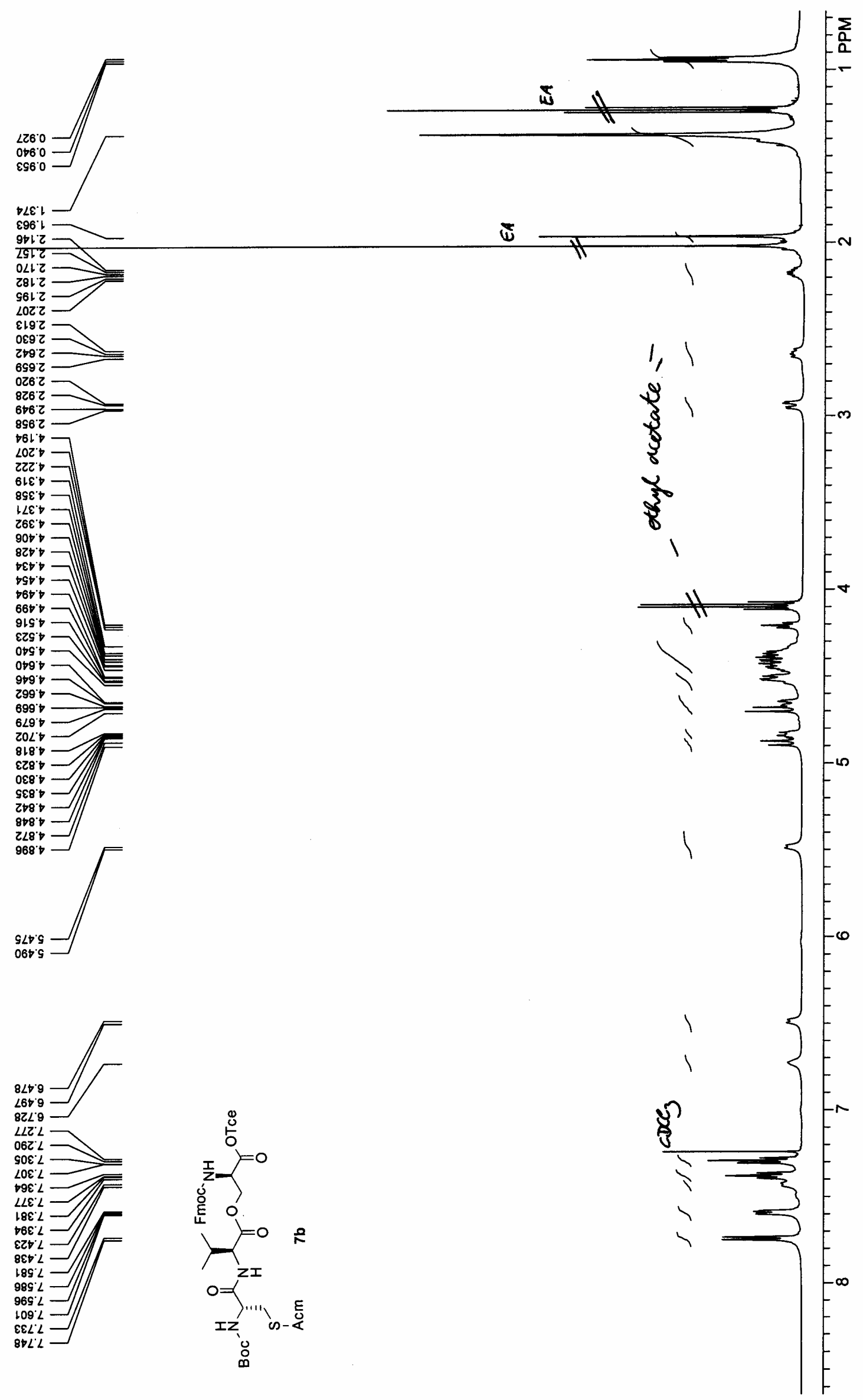
$789^{\circ} \angle 1$

$\varepsilon \varepsilon 0 \%$

9เZะZ

Z9Z' 82

$\rightarrow 1 S 0 \varepsilon$

$696+\varepsilon$

$9 z 2 \downarrow t$

$9 \angle 0^{\circ} \angle O$

$00 乙 \varepsilon \varsigma$ 

$126<5$

tos' 59

$692 \angle 9$

$8 Z L '+L$

$\downarrow Z 2 \cdot 08$

$80 z^{\prime}+6$

$2 \angle 661 t$

090'sZ।

tol $2 Z 1$

$Z L L \angle Z L$

乙๕乙レเレ
$6 Z 9 \varepsilon ヤ レ$

$6 Z 9 \varepsilon+1$

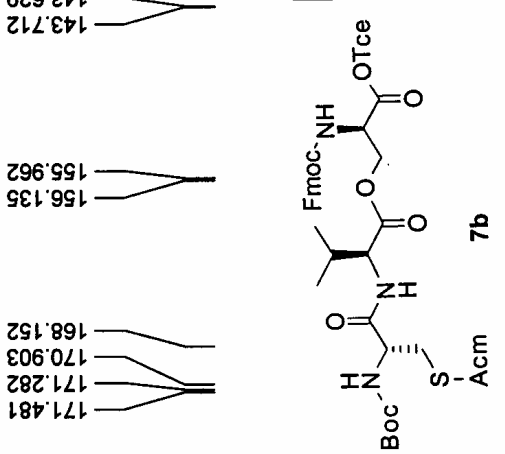

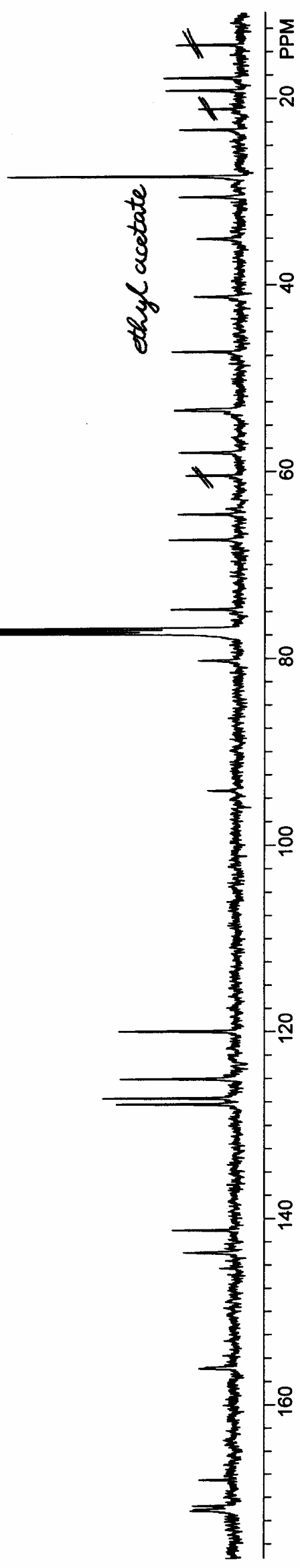




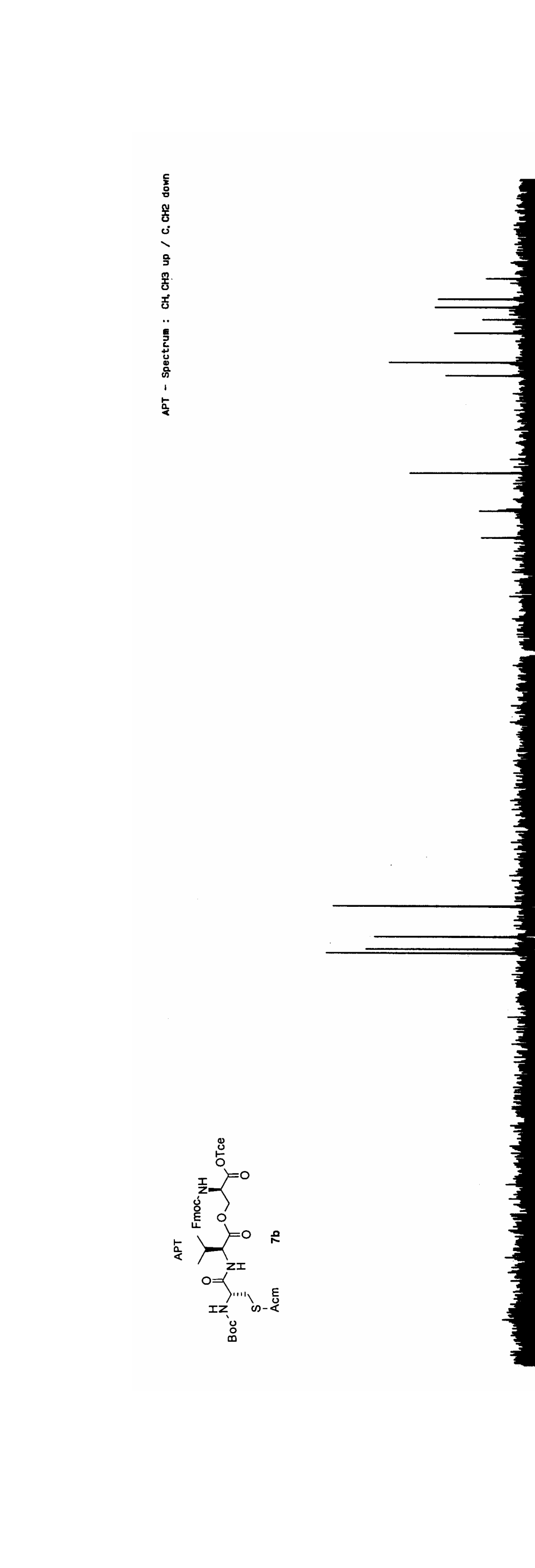




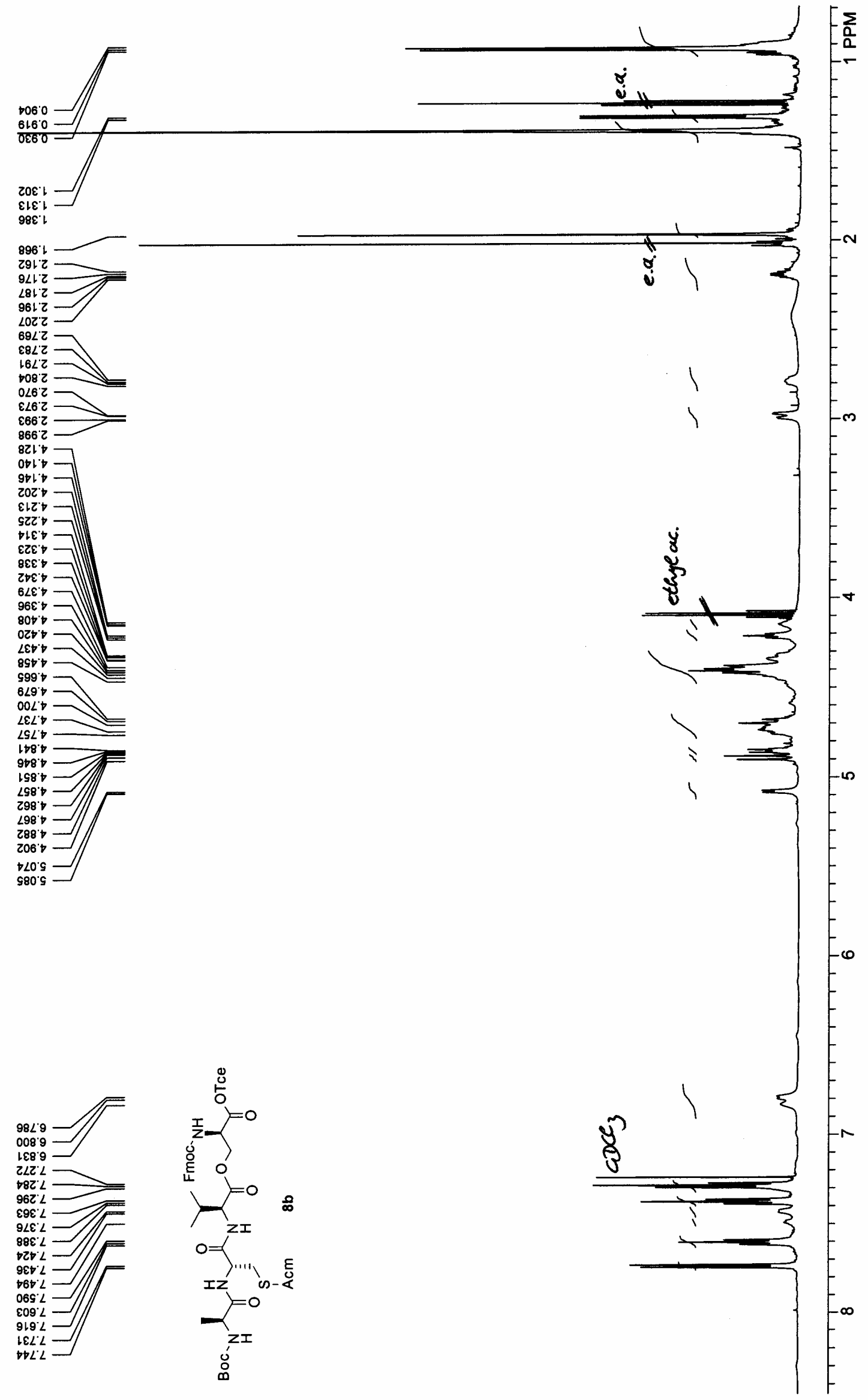


$\nabla \varepsilon 9^{\circ} L$

$\rightarrow 91 \cdot 81$

$286{ }^{\circ} 8 \mathrm{~L}$

$6 乙 乙 \varepsilon 乙$

$9 \varepsilon 乙 .82$

StS'0 0

$\varepsilon 88^{\circ} \varepsilon \varepsilon$

$08+1$

$800^{\circ}$

\&ZS'0S

019 'Z

00є' $\varsigma$

$098^{\circ} \angle S^{\circ}$

SLLto

SLL' $\angle 9$

$0 \angle 9 \circ \angle-$

$y^{m}$

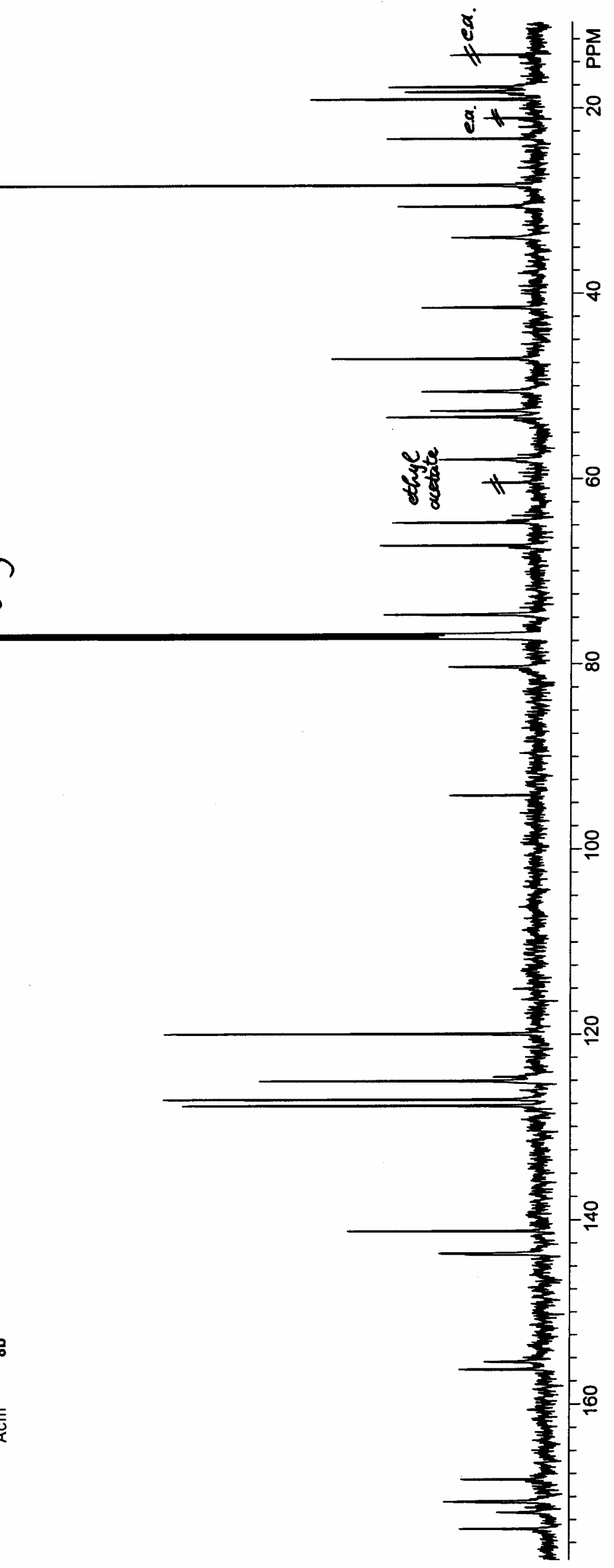

086 ' 6 ᄂ

$16 S^{\circ} \sqcup Z L$

SSO'SZL

$9 \angle 0 . \angle Z L$

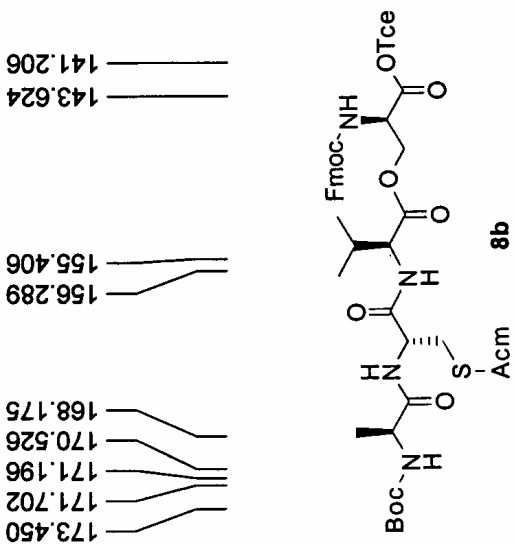




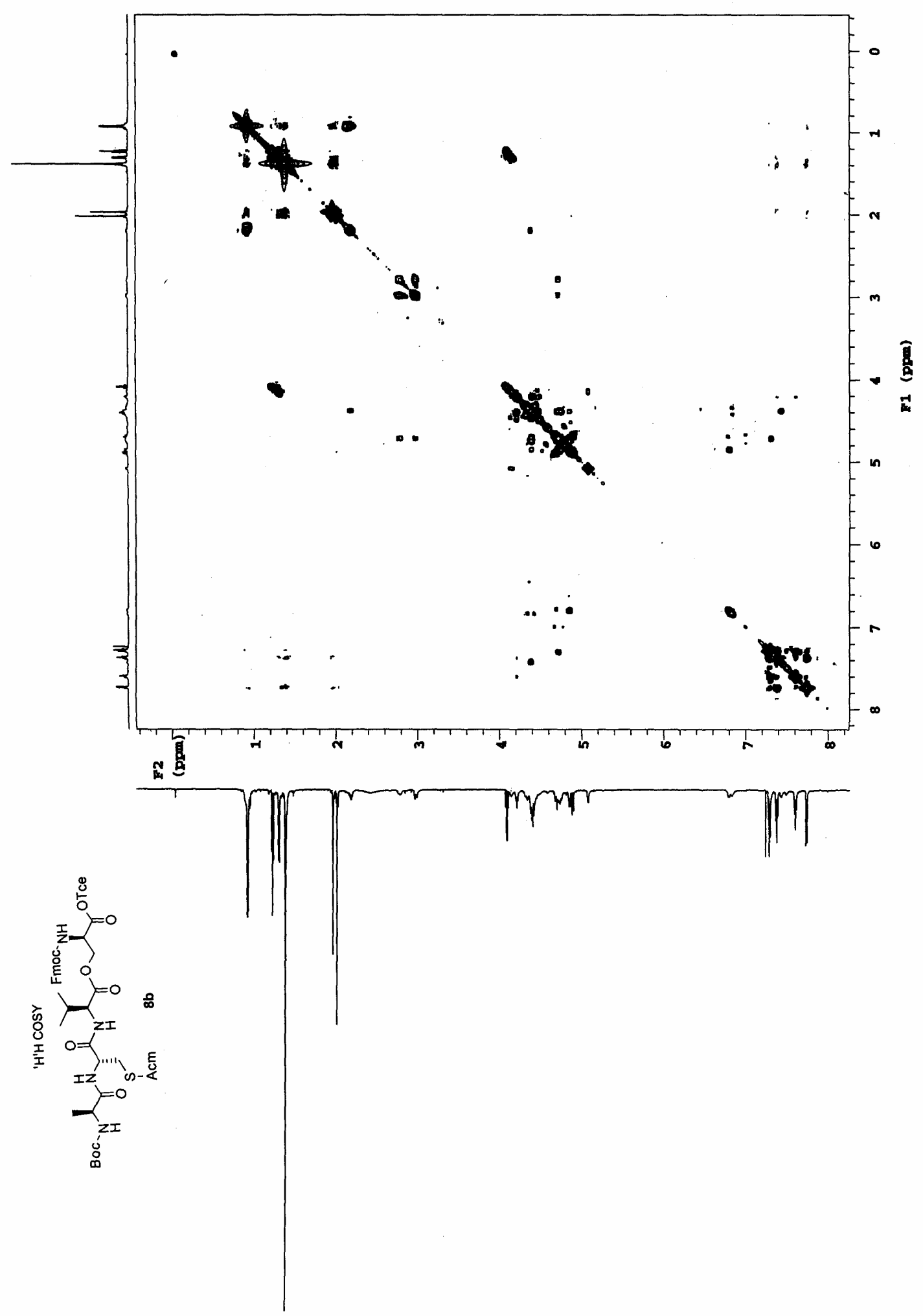




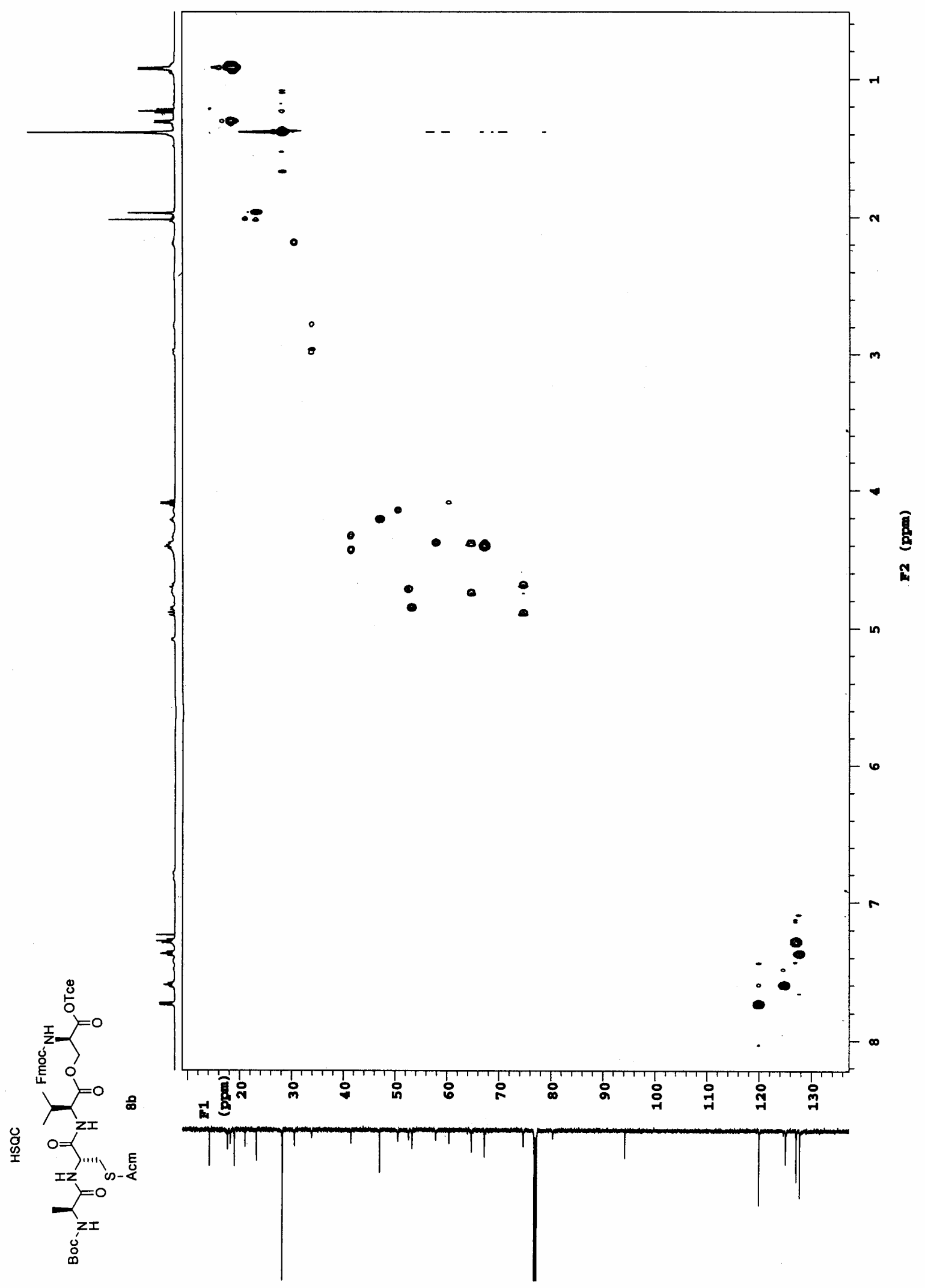




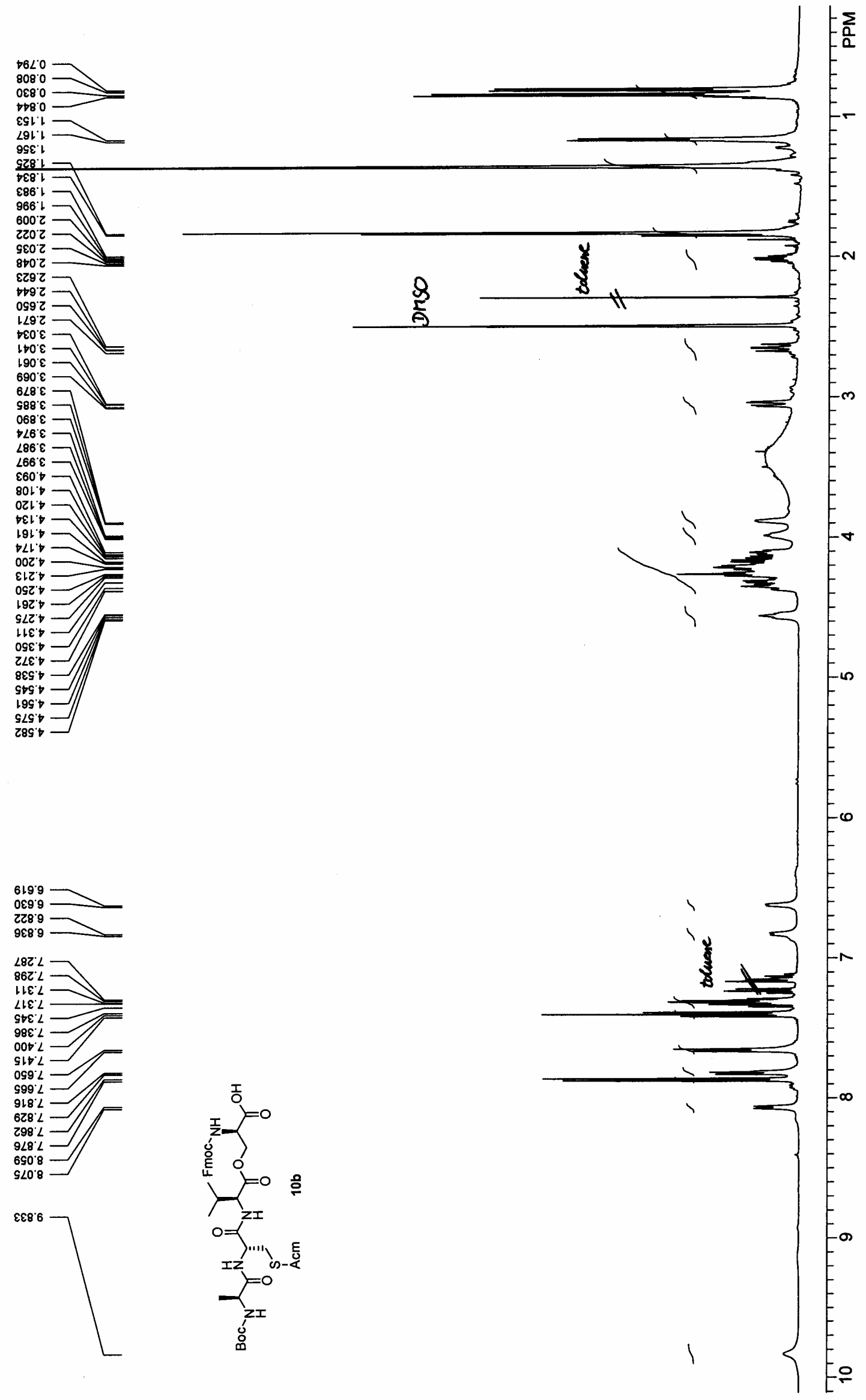


$z z t \cdot 8 z$

เร8 62

$\frac{8}{5}$

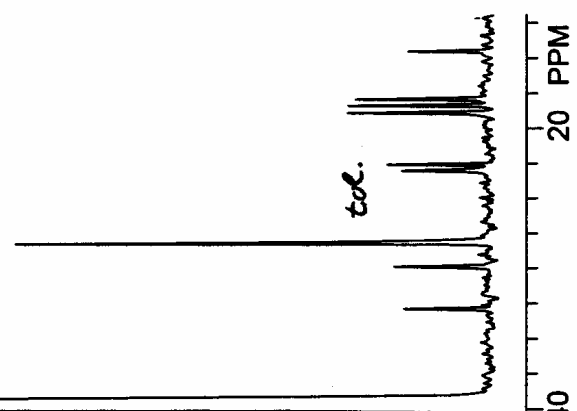

689.9

ElL $6 t$

SZ8.L

$\angle L D S$

825.25

$\multimap 6 \varepsilon 09$

61959

$6+0.8 L$

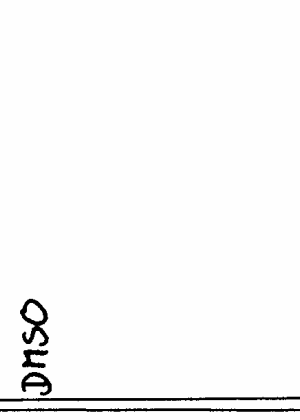

210021

$\checkmark 6 Z 121$

$\angle S O$ SZL

$866^{\circ} 92$

$\varepsilon L Z \angle Z$

$8 E S^{\circ} \angle Z L$

घ98.8Z -
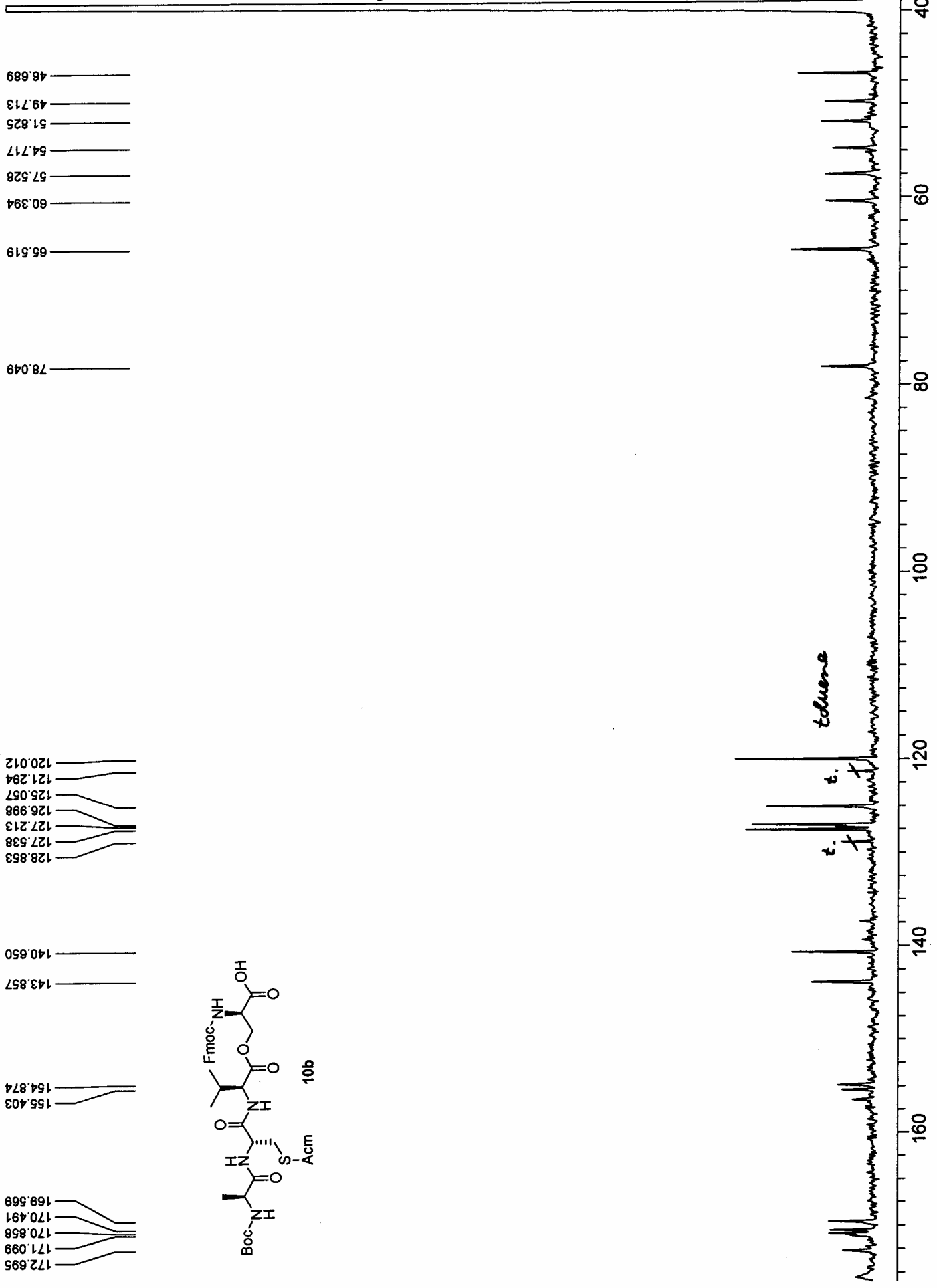


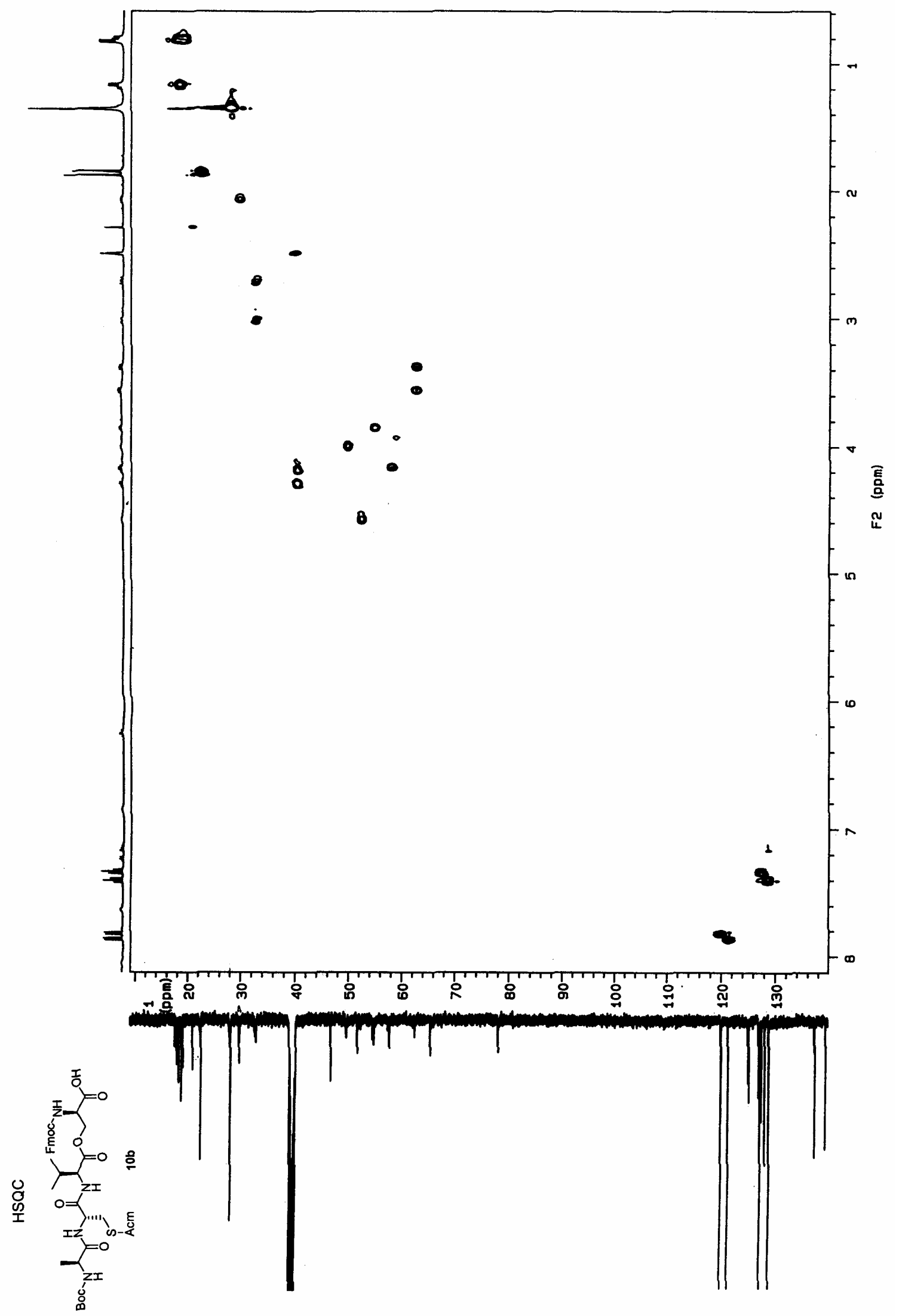


5180

-

s9l.

$8<1 \div$

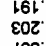

เse' 12

(t)

$\angle \circ 0^{\circ}$
910

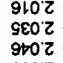

${ }_{9002}$

8902 -

$1602=$

Es9z

$099 \mathrm{z}=$

6992
9992
$2692 z$
8692

96827

9062

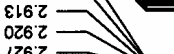

$\angle 262=1$

886

$\angle 66 \varepsilon$
$900 \%$

$900 \%$
$\varepsilon 0^{\circ} \downarrow$

zelt =

602,7

sLz

$\angle 0 \varepsilon, b$
SLE

$\varepsilon \varepsilon \varepsilon ;$

Ese

ozt

Gest

EॄS

ars

Lsst

gosiv

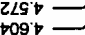

8096

$\varepsilon \varepsilon 9 \triangleright$ 二

$\angle r 9 ;=1$

os9' $t$

s99', 二

98 '



996.

เEO'S

$250 \circ$
$\square L O S$
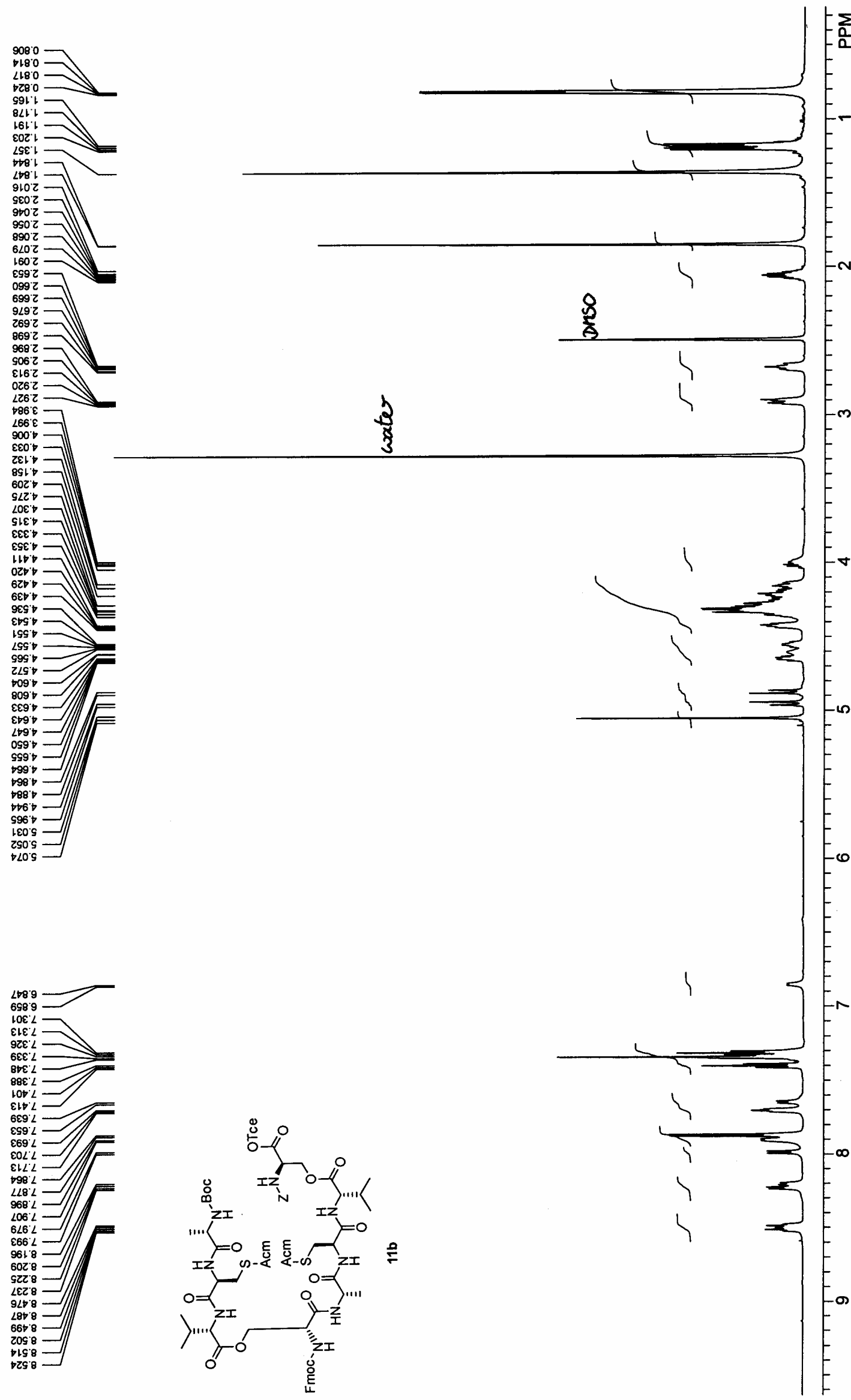

$\sqrt[3]{3}$

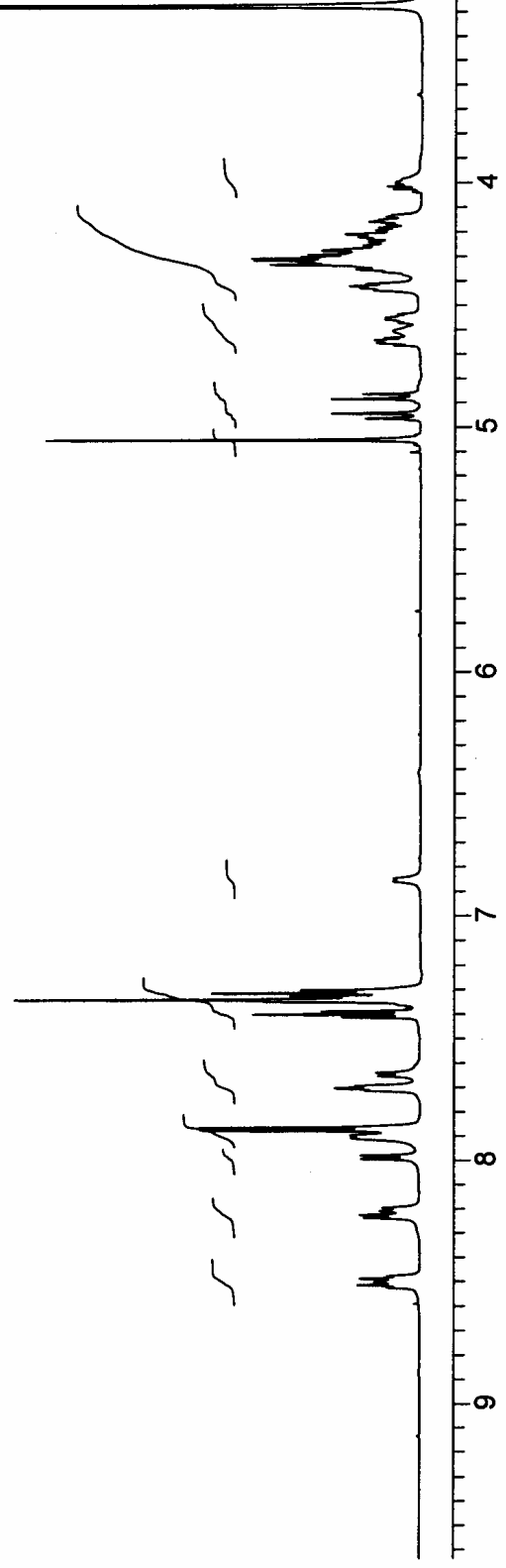


$92+\angle t$

9ts $\angle 1$

$\begin{array}{lll}181 & 81 \\ \varepsilon \varepsilon \rightarrow 81 & \end{array}$

$8 \varepsilon+81$

$80 L 81$

sst $z Z$

560.82

$\angle 26.6 Z$

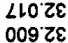

8
$\frac{8}{4}$

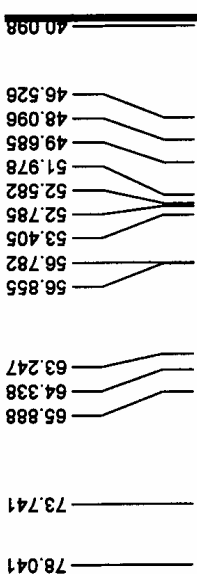

$82 L \div 6$
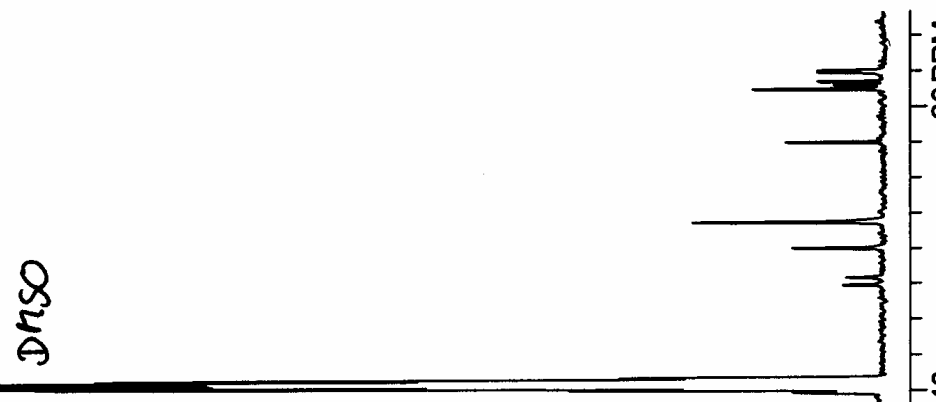

$1+0.8<$

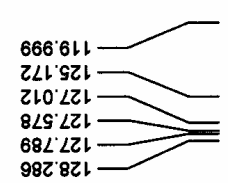

$999^{\circ} 9 \varepsilon\llcorner$

$629 \cdot 001$

899.Etน
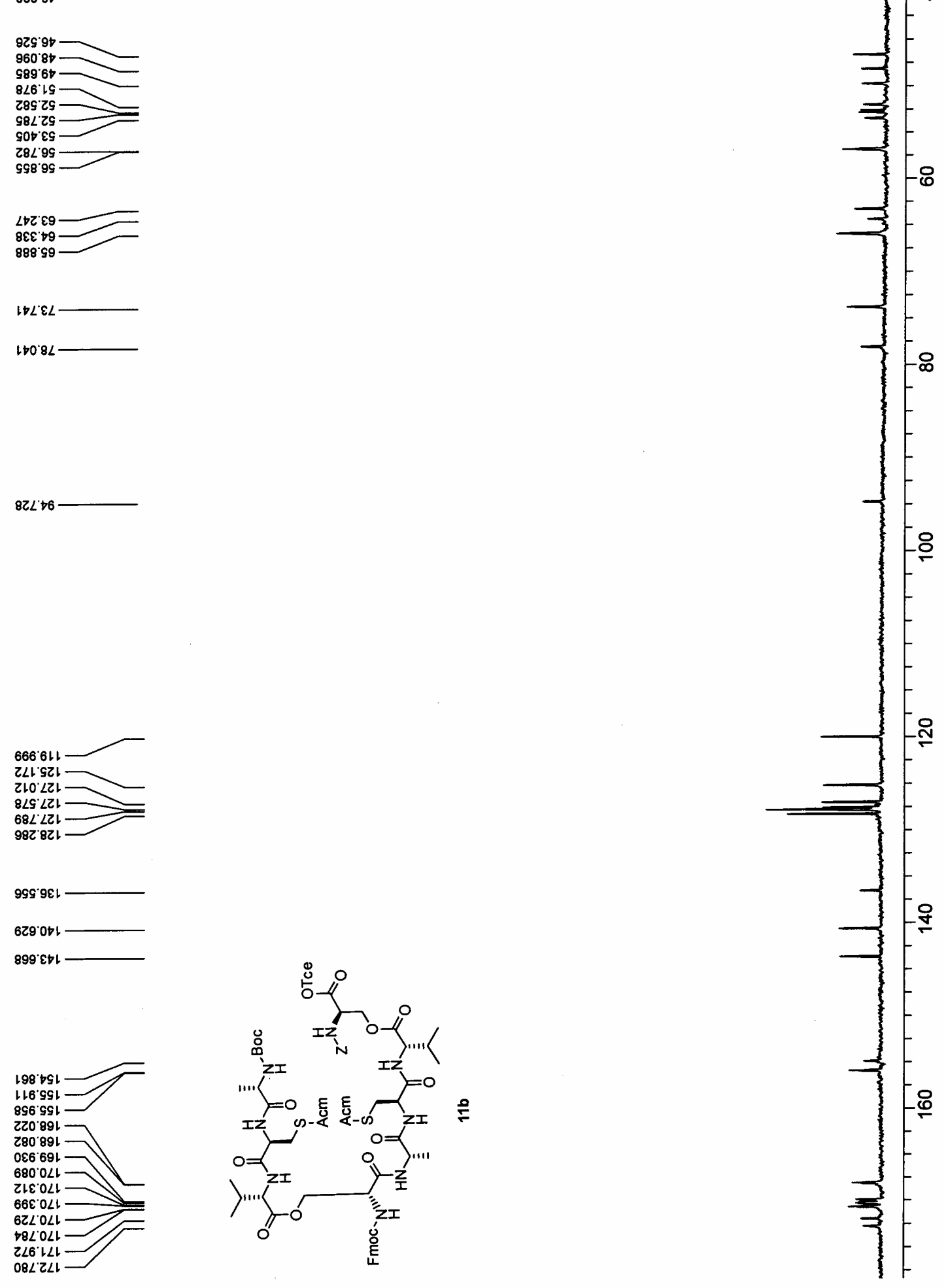


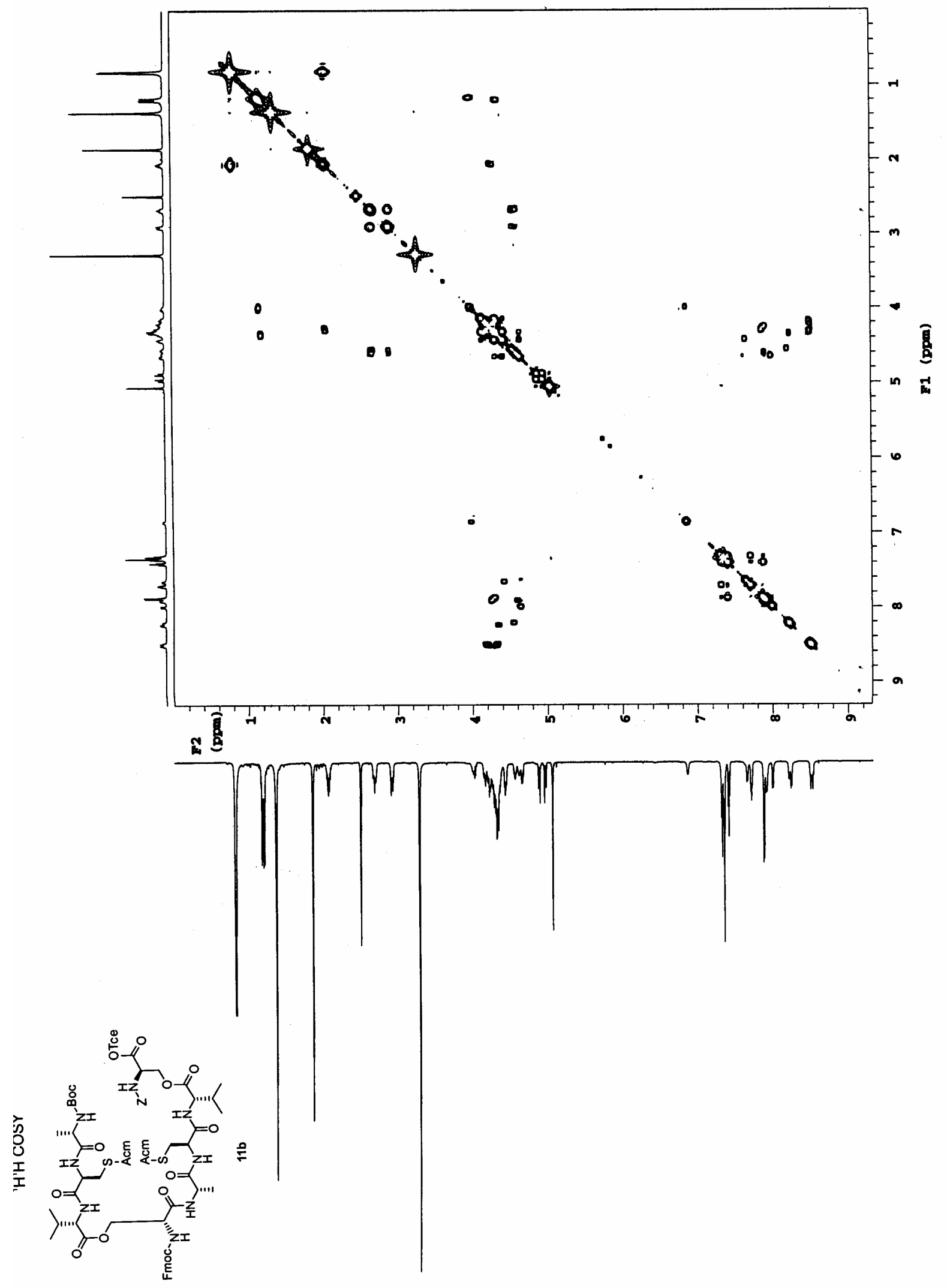




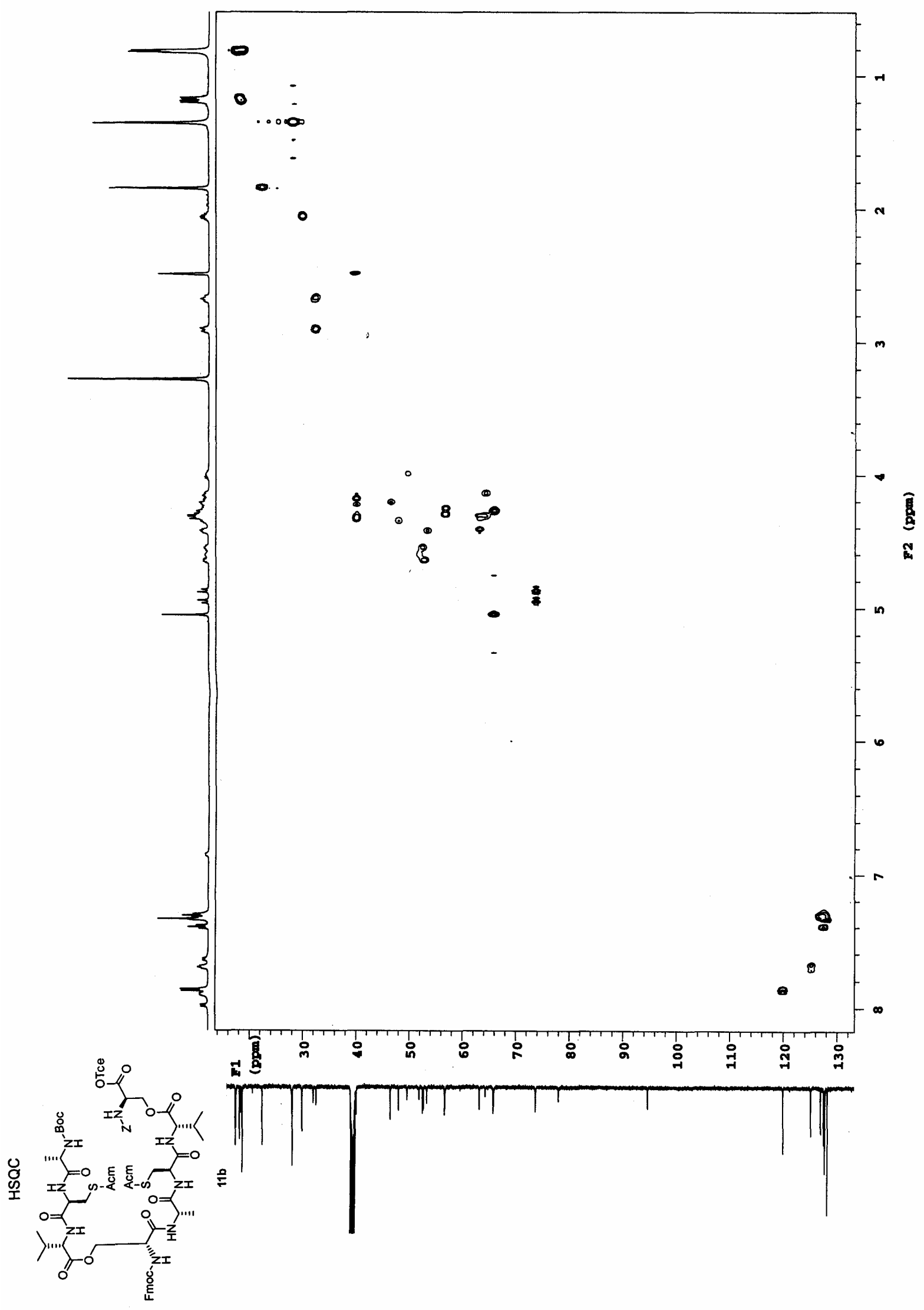




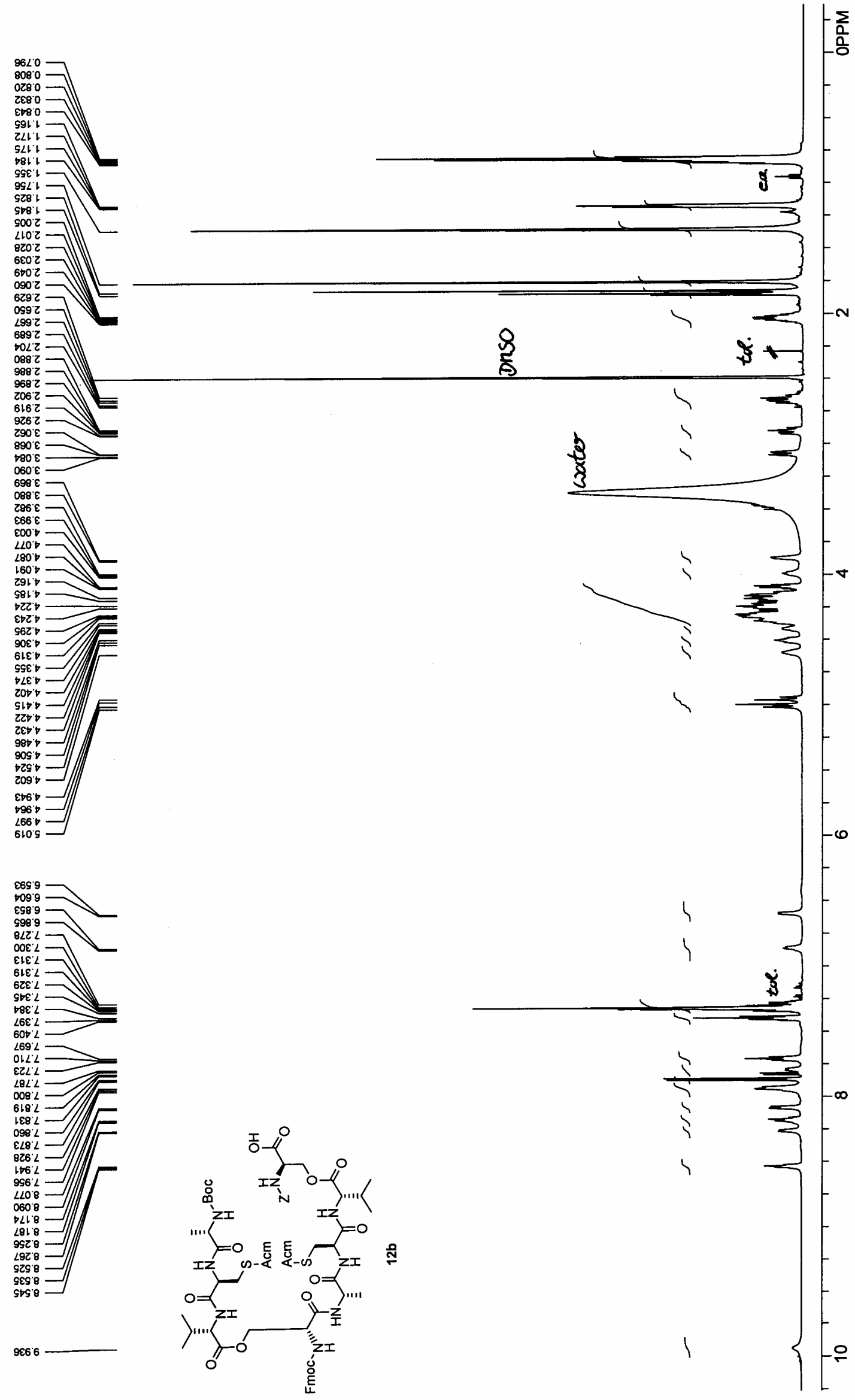



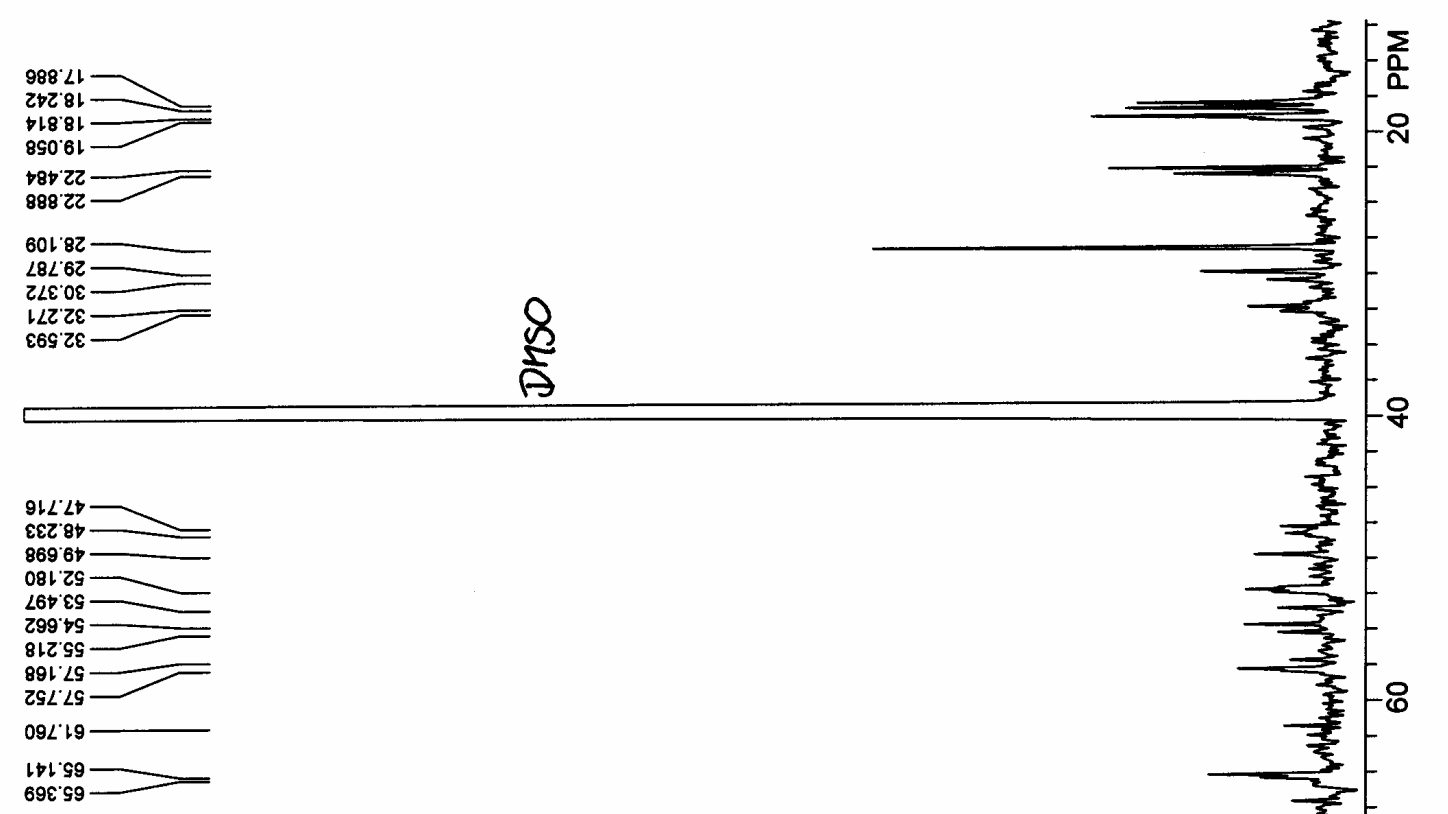

$2 \varepsilon 0^{\circ} 8 L$

OZ6.6IL

$\angle L Z \mid Z Z$

$\forall E 1+2$

$S 6 L \angle Z L$
$\square \angle S \cdot L Z L$

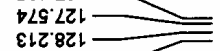

z\&8 $82 \downarrow$

$89 \varepsilon \cdot \angle \varepsilon L$

6t\&. $6 \varepsilon$

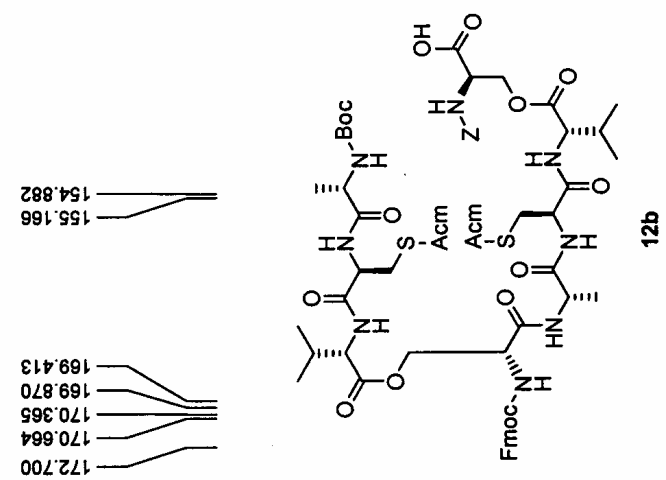

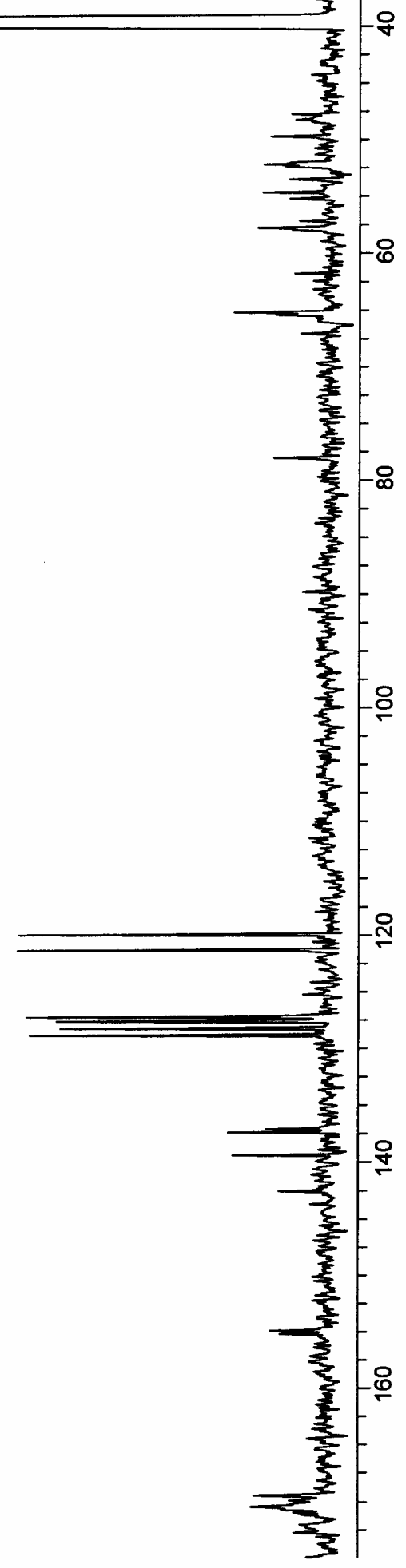




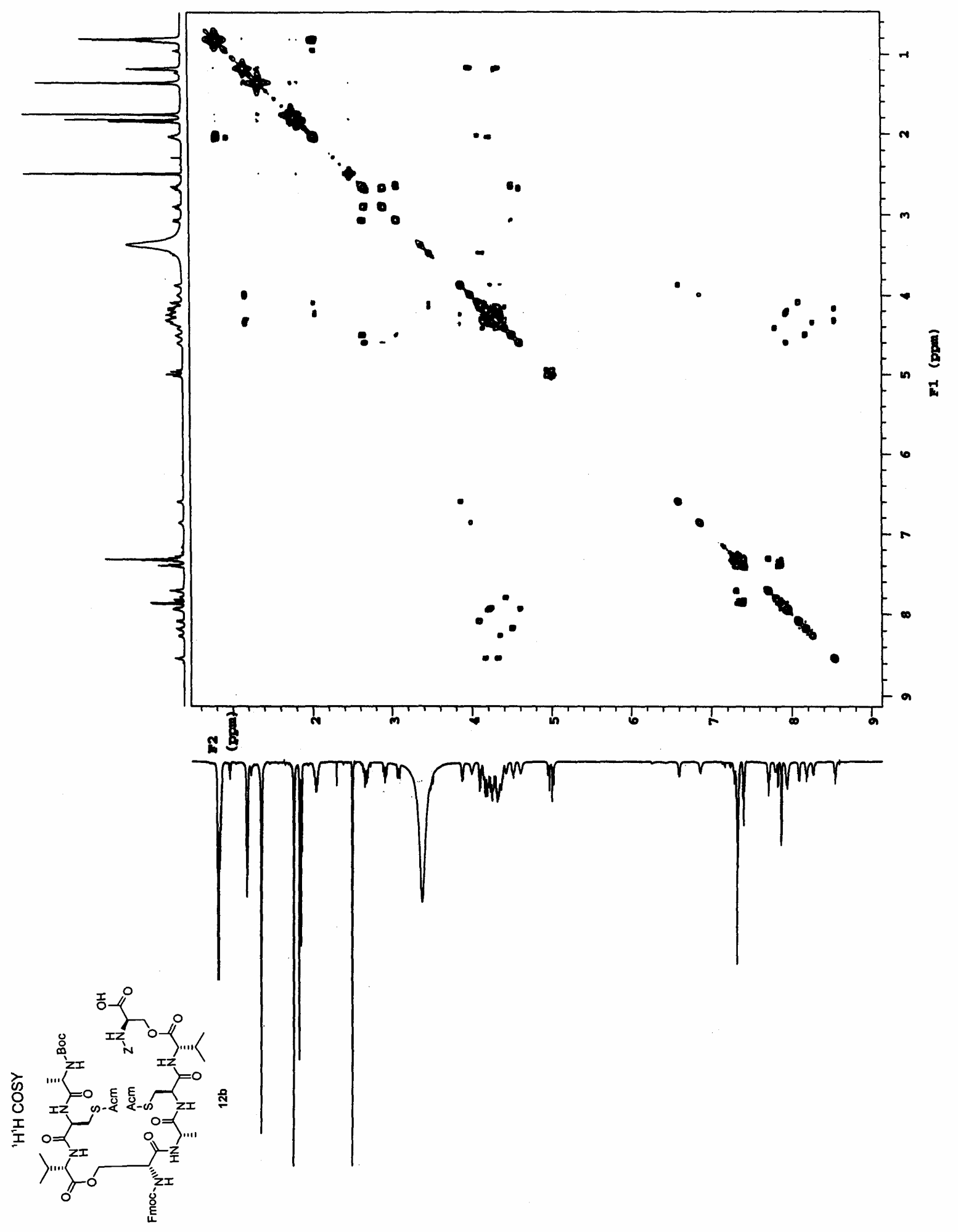




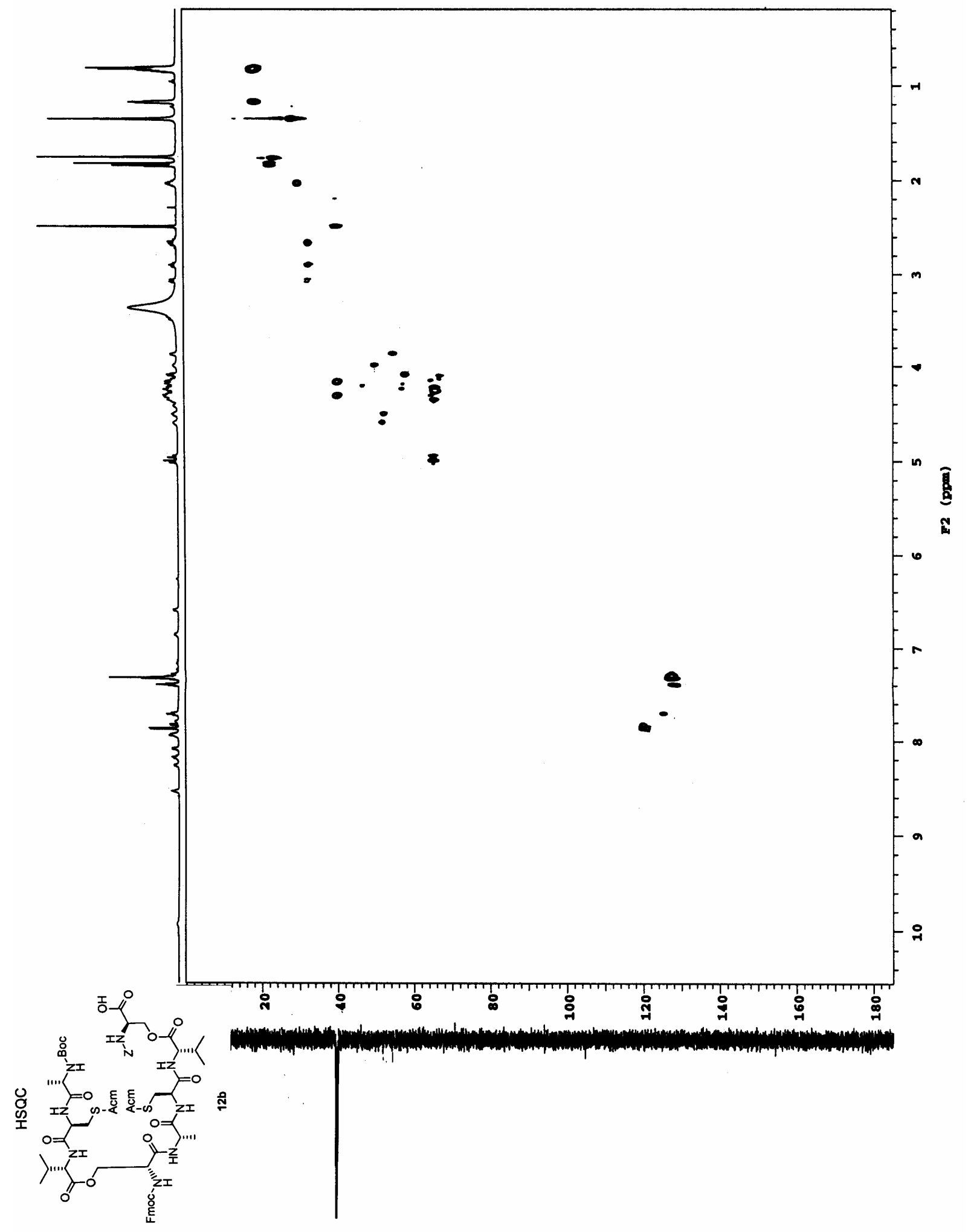



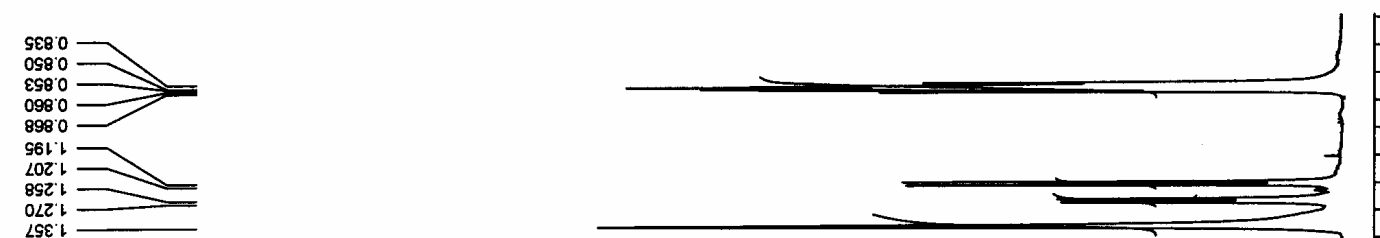

$\angle S E$

8502
9002
9402

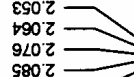

8002

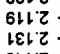

lei. $2=$

ตre

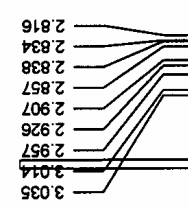

$266^{\circ} \varepsilon$
$800^{\circ} \circ$

sil, =

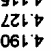

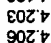

9oz 6

每252

OLZ

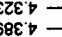

10t'

9tt

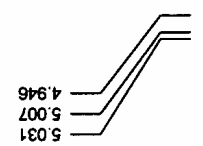

$\frac{8}{\text { ลิ }}$

$\frac{3}{3}$
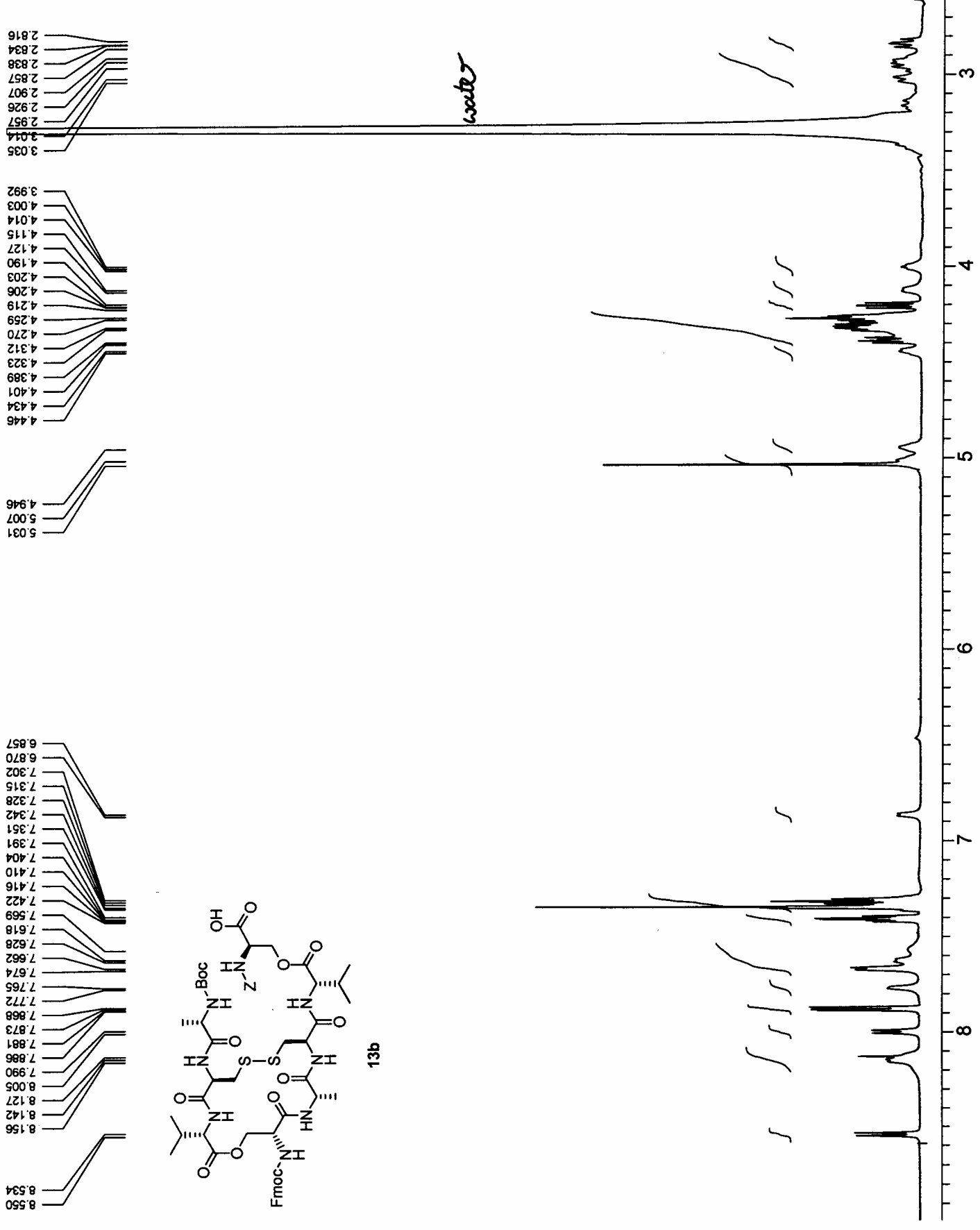
199.

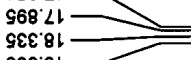

$\$ \varepsilon \varepsilon^{\prime} 81$
$808^{\circ} 8 \mathrm{~L}$

$\angle 90^{\circ} 82$
$\varepsilon \angle 8.82-\square$
$\varepsilon \multimap 9^{\circ} 0 \varepsilon$

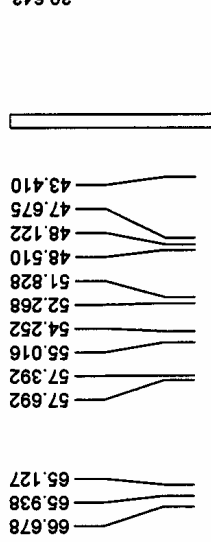

$8269 \square$

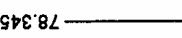

$\angle 26.611$

Z8Z เZレ

SOZ $\angle Z L$

$8 \angle S^{\prime} \angle Z L$

GZZ $8 Z 1$

$90^{\circ} 821$

૬ $9 \varepsilon: \angle \varepsilon\llcorner$

9รE' $6 \varepsilon L$

sıs'th
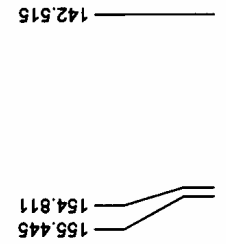

$081 \cdot 69 \mathrm{~L}$

\&et $69 \mathrm{l}$

$800.0 \angle L$

SZO L LL

$8 L 2 \downarrow L 1=$

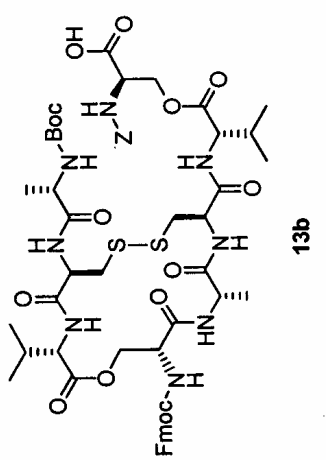

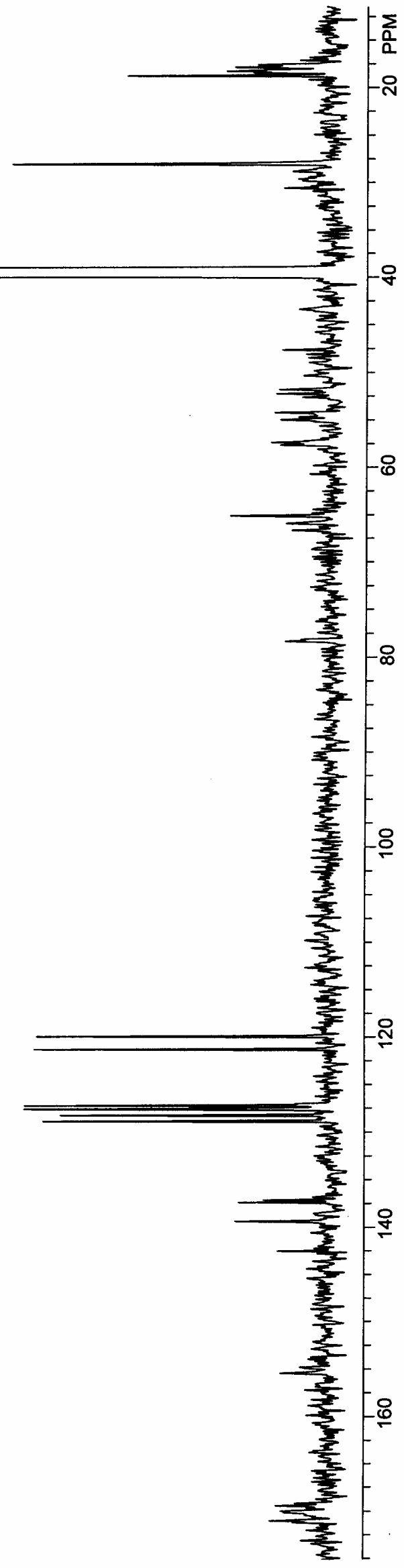




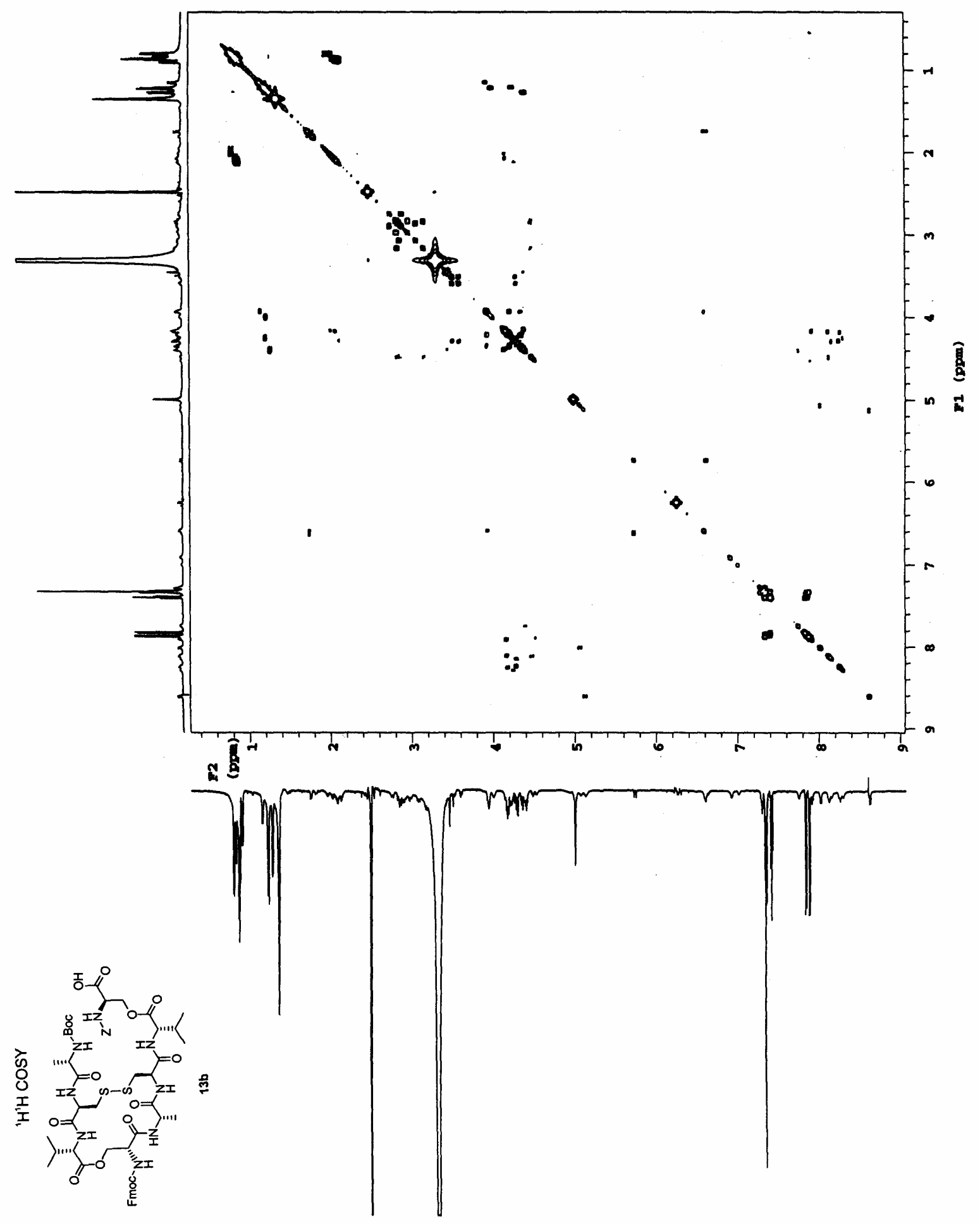




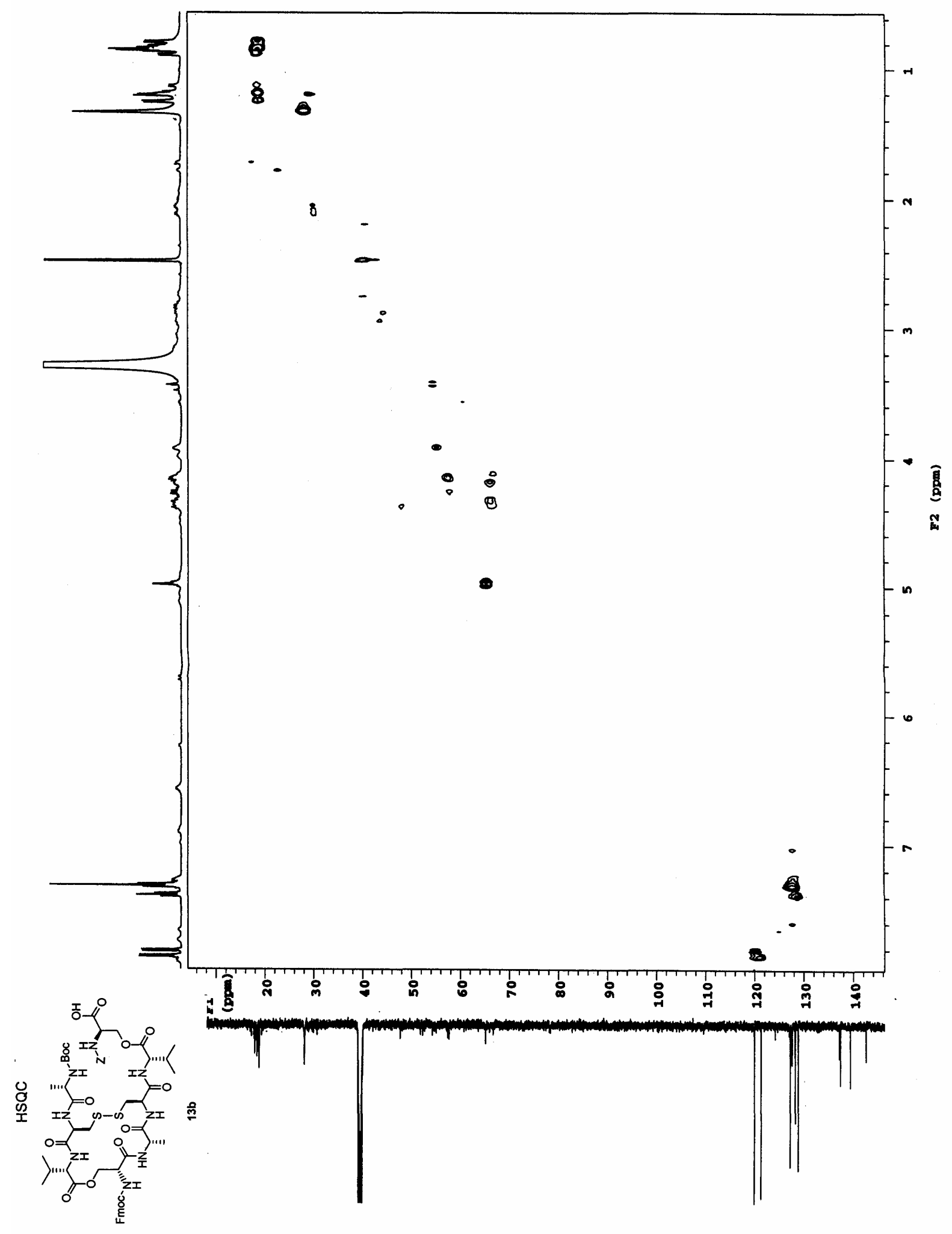



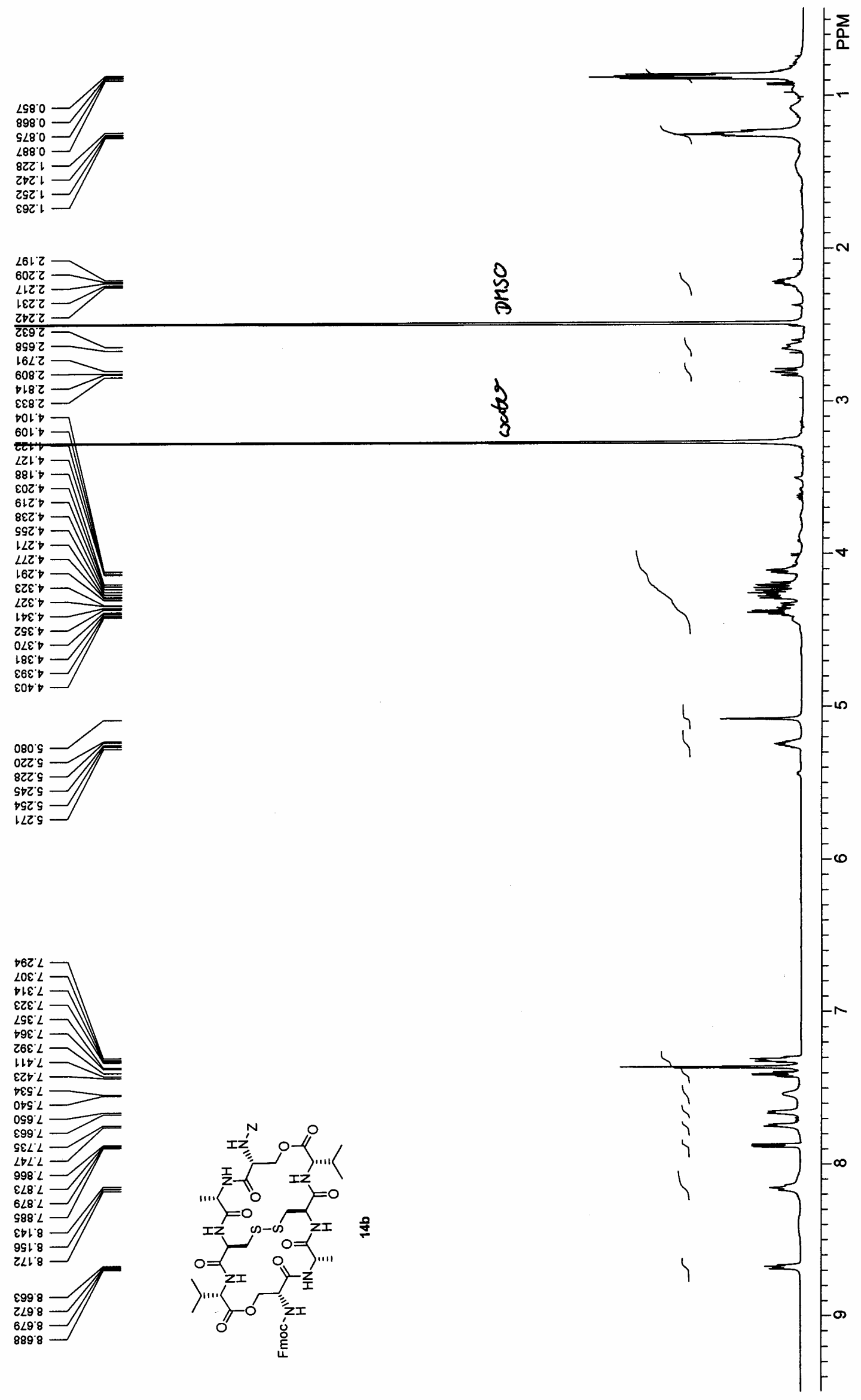
590.81

$\angle 0 Z$ ' $8 \mathrm{~L}$

$988^{\circ} 8 \mathrm{~L}$

$9 \varepsilon 6.81$

¿८००६

0

$\frac{5}{9}$

tro't

$509{ }^{\circ} 9 t$

દE० 87

$102 \mathrm{ZS}$

ZE9 $\downarrow S$

962.89

เSE' 89

11899

$\downarrow \angle 8.99$

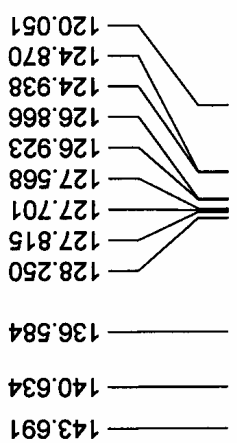

เ69'๕ヤ
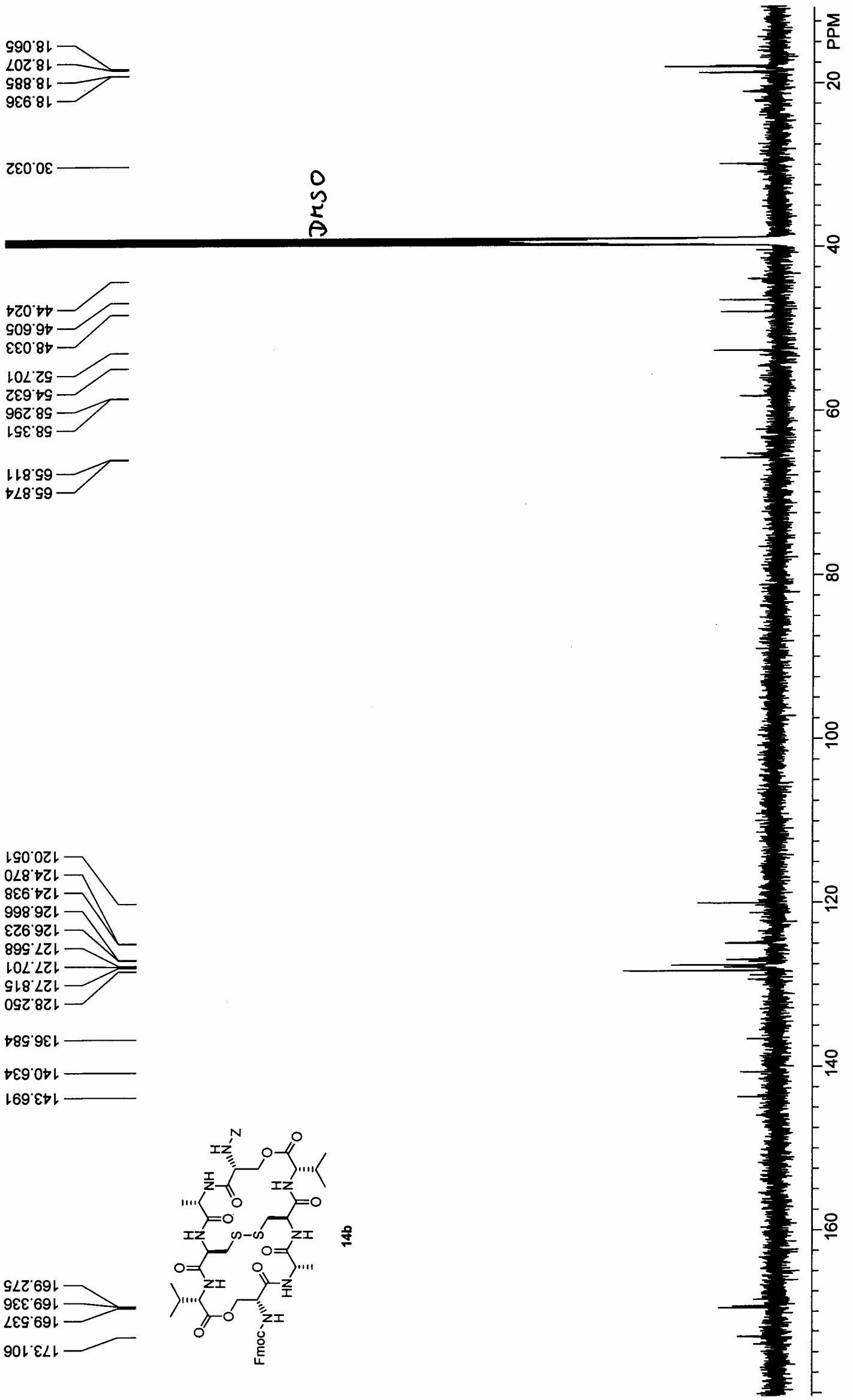


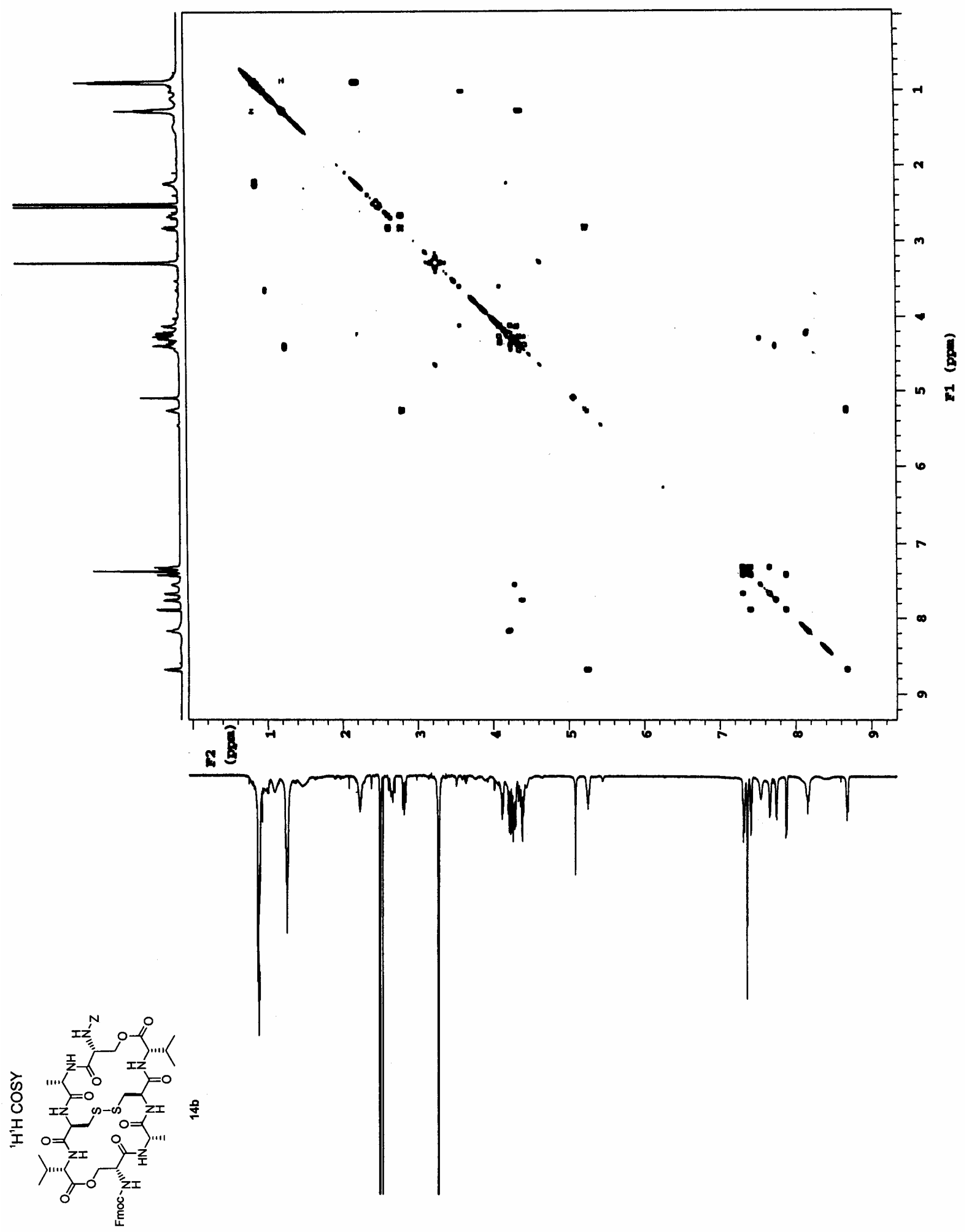




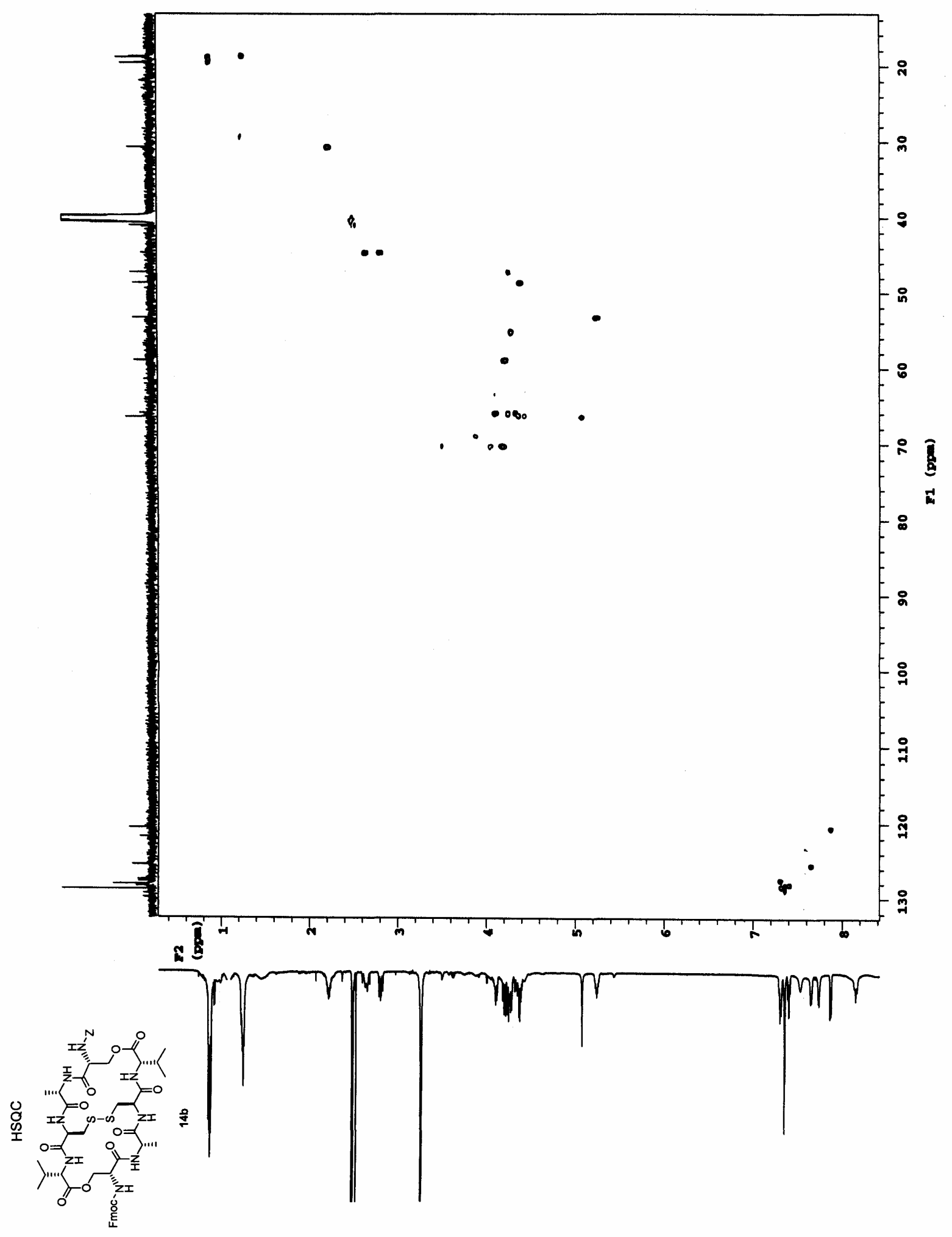



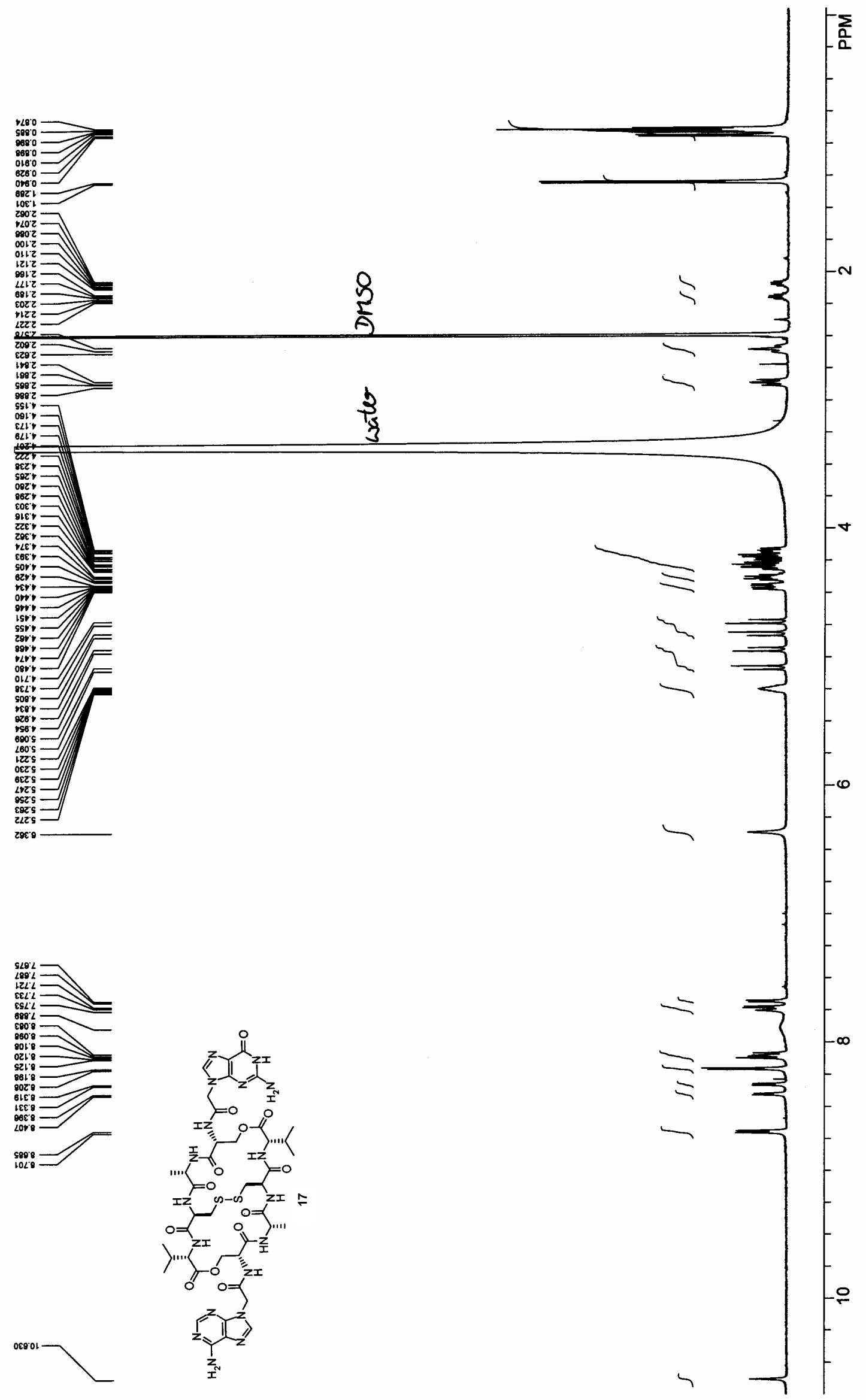

$\frac{1}{3}$ 


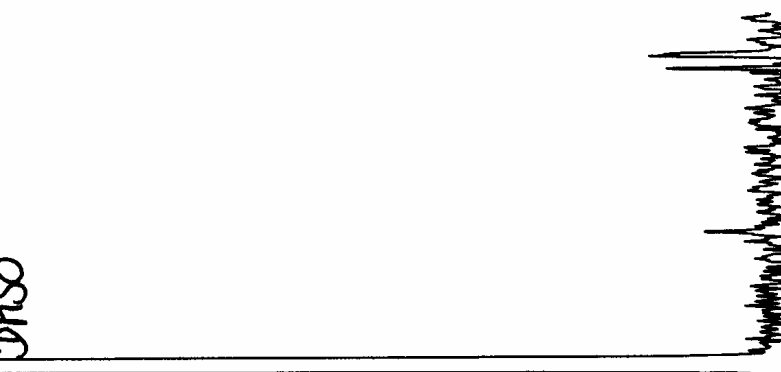

$\angle 8 Z \circ \mathcal{-}$

ล

608.

$9865 t=$

$\checkmark 66^{\circ} \angle t$

ZOL'Zs

$6 เ \varepsilon \cdot 89$

$108+79$

GLI'8Lt
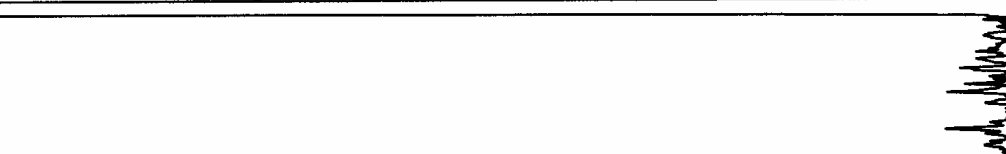

9

-

$1608 \varepsilon 1$

เฉo.09เ

$6 \varepsilon S^{\prime} \angle 9 L$

$689^{\circ} 69 \mathrm{l}$

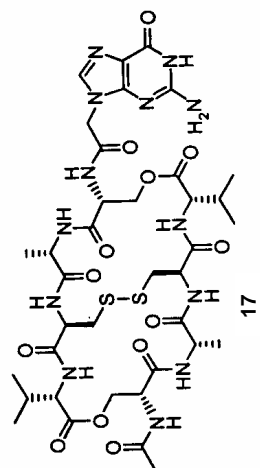

$99 z \varepsilon<\llcorner$

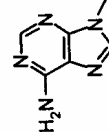




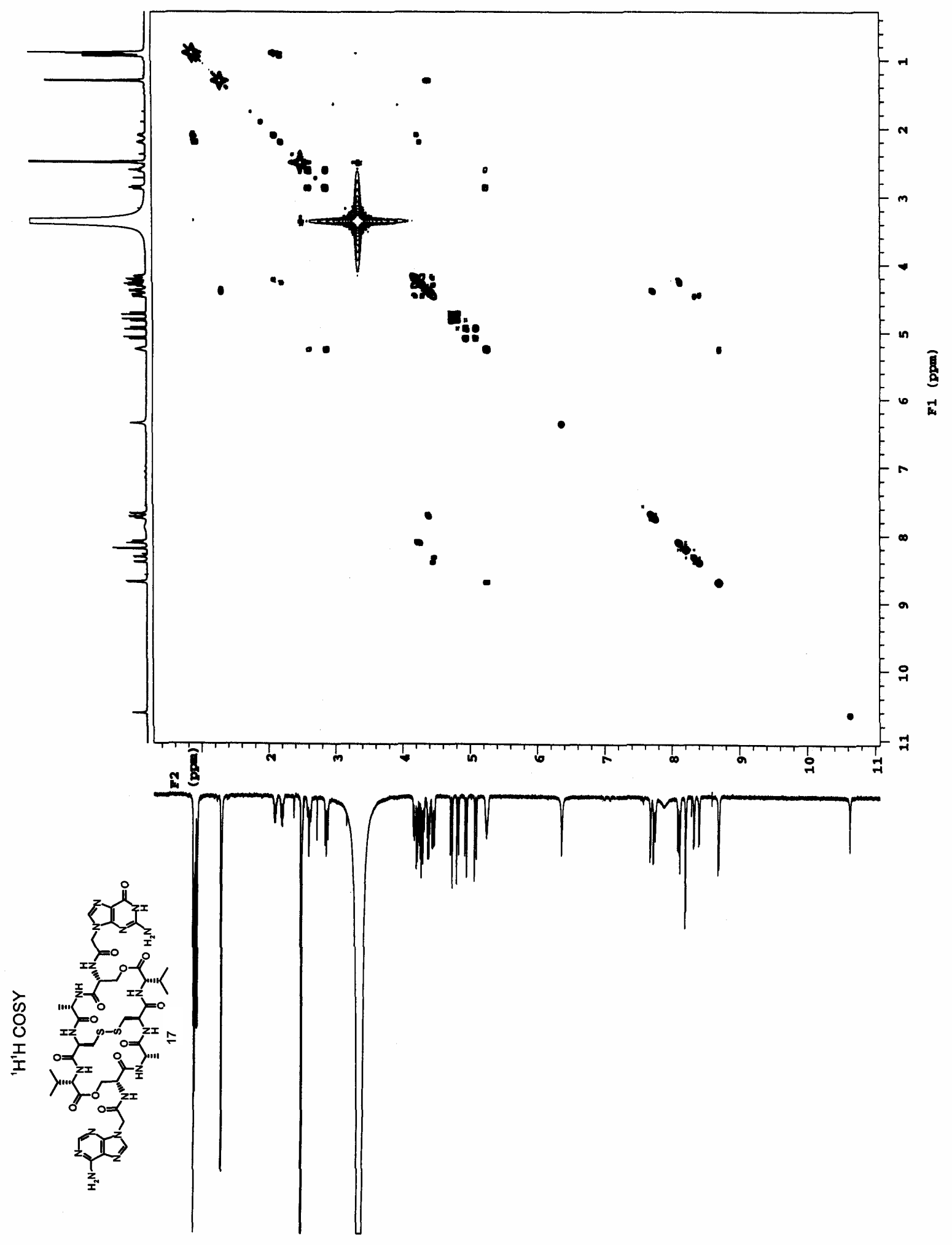




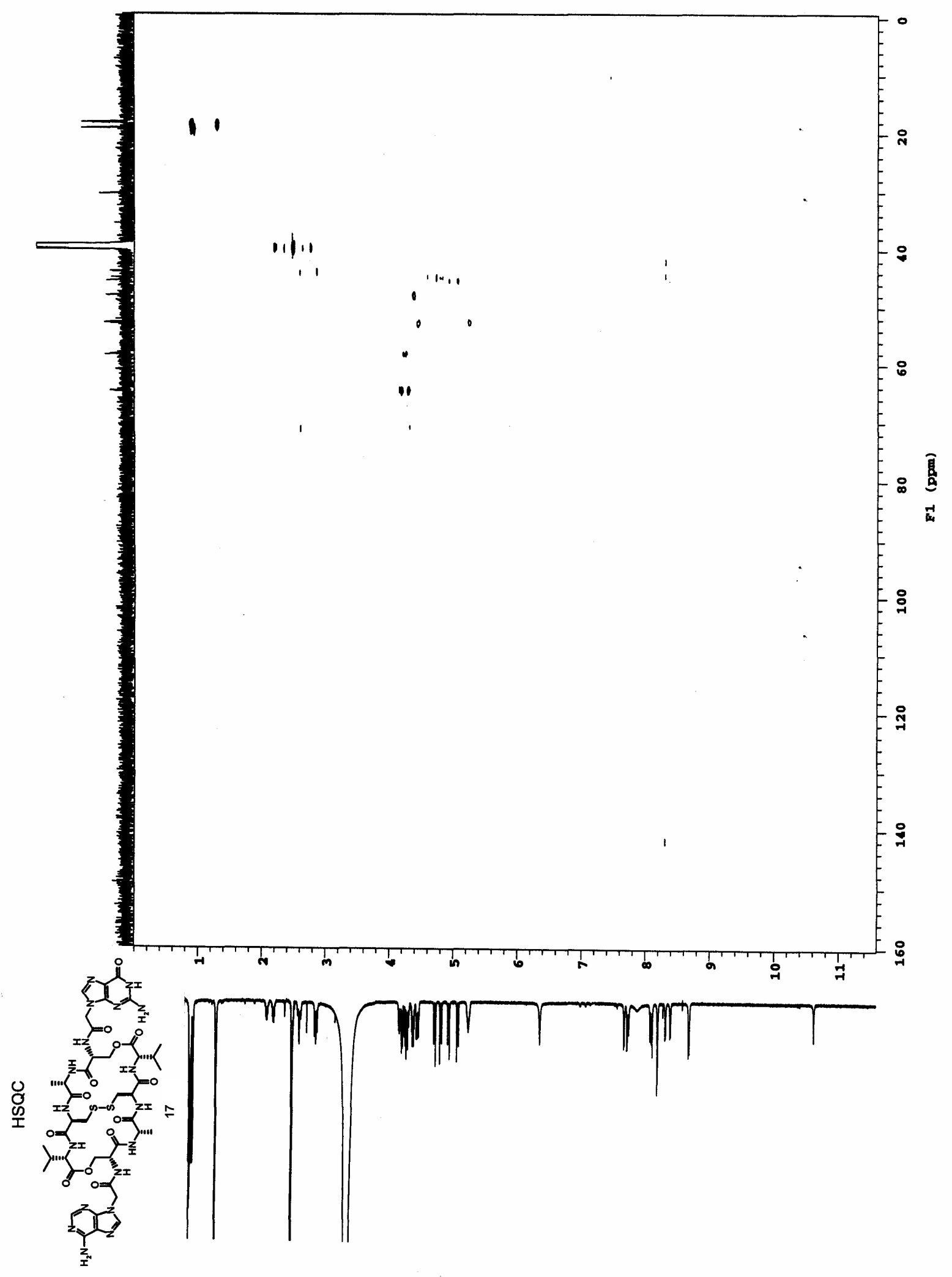

